\title{
The primordial nucleus of comet 67P/Churyumov-Gerasimenko
}

\author{
B. J. R. Davidsson ${ }^{1,2}$, H. Sierks ${ }^{3}$, C. Güttler ${ }^{3}$, F. Marzari ${ }^{4}$, M. Pajola ${ }^{5}$, H. Rickman ${ }^{1,6}$, M. F. A'Hearn ${ }^{7,8}$, A.-T. Auger ${ }^{9}$, \\ M. R. El-Maarry ${ }^{10}$, S. Fornasier ${ }^{11}$, P. J. Gutiérrez ${ }^{12}$, H. U. Keller ${ }^{13}$, M. Massironi ${ }^{5,14}$, C. Snodgrass ${ }^{15}$, J.-B. Vincent ${ }^{3}$, \\ C. Barbieri ${ }^{16}$, P. L. Lamy ${ }^{17}$, R. Rodrigo ${ }^{18,19}$, D. Koschny ${ }^{20}$, M. A. Barucci ${ }^{11}$, J.-L. Bertaux ${ }^{21}$, I. Bertini ${ }^{5}$, \\ G. Cremonese ${ }^{22}$, V. Da Deppo ${ }^{23}$, S. Debei ${ }^{24}$, M. De Cecco ${ }^{25}$, C. Feller ${ }^{11,26}$, M. Fulle ${ }^{27}$, O. Groussin' ${ }^{9}$, S. F. Hviid ${ }^{28}$, \\ S. Höfner ${ }^{3}$, W.-H. Ip ${ }^{29}$, L. Jorda ${ }^{17}$, J. Knollenberg ${ }^{28}$, G. Kovacs ${ }^{3}$, J.-R. Kramm ${ }^{3}$, E. Kührt ${ }^{28}$, M. Küppers ${ }^{30}$, \\ F. La Forgia ${ }^{16}$, L. M. Lara ${ }^{12}$, M. Lazzarin ${ }^{16}$, J. J. Lopez Moreno ${ }^{12}$, R. Moissl-Fraund ${ }^{30}$, S. Mottola ${ }^{28}$, G. Naletto ${ }^{31}$, \\ N. Oklay ${ }^{3}$, N. Thomas ${ }^{10}$, and C. Tubiana ${ }^{3}$ \\ (Affiliations can be found after the references)
}

Received 15 July 2015 / Accepted 15 March 2016

\begin{abstract}
Context. We investigate the formation and evolution of comet nuclei and other trans-Neptunian objects (TNOs) in the solar nebula and primordial disk prior to the giant planet orbit instability foreseen by the Nice model.

Aims. Our goal is to determine whether most observed comet nuclei are primordial rubble-pile survivors that formed in the solar nebula and young primordial disk or collisional rubble piles formed later in the aftermath of catastrophic disruptions of larger parent bodies. We also propose a concurrent comet and TNO formation scenario that is consistent with observations.

Methods. We used observations of comet 67P/Churyumov-Gerasimenko by the ESA Rosetta spacecraft, particularly by the OSIRIS camera system, combined with data from the NASA Stardust sample-return mission to comet 81P/Wild 2 and from meteoritics; we also used existing observations from ground or from spacecraft of irregular satellites of the giant planets, Centaurs, and TNOs. We performed modeling of thermophysics, hydrostatics, orbit evolution, and collision physics.

Results. We find that thermal processing due to short-lived radionuclides, combined with collisional processing during accretion in the primordial disk, creates a population of medium-sized bodies that are comparably dense, compacted, strong, heavily depleted in supervolatiles like CO and $\mathrm{CO}_{2}$; they contain little to no amorphous water ice, and have experienced extensive metasomatism and aqueous alteration due to liquid water. Irregular satellites Phoebe and Himalia are potential representatives of this population. Collisional rubble piles inherit these properties from their parents. Contrarily, comet nuclei have low density, high porosity, weak strength, are rich in supervolatiles, may contain amorphous water ice, and do not display convincing evidence of in situ metasomatism or aqueous alteration. We outline a comet formation scenario that starts in the solar nebula and ends in the primordial disk, that reproduces these observed properties, and additionally explains the presence of extensive layering on 67P/Churyumov-Gerasimenko (and on 9P/Tempel 1 observed by Deep Impact), its bi-lobed shape, the extremely slow growth of comet nuclei as evidenced by recent radiometric dating, and the low collision probability that allows primordial nuclei to survive the age of the solar system. Conclusions. We conclude that observed comet nuclei are primordial rubble piles, and not collisional rubble piles. We argue that TNOs formed as a result of streaming instabilities at sizes below $\sim 400 \mathrm{~km}$ and that $\sim 350$ of these grew slowly in a low-mass primordial disk to the size of Triton, Pluto, and Eris, causing little viscous stirring during growth. We thus propose a dynamically cold primordial disk, which prevented mediumsized TNOs from breaking into collisional rubble piles and allowed the survival of primordial rubble-pile comets. We argue that comets formed by hierarchical agglomeration out of material that remained after TNO formation, and that this slow growth was a necessity to avoid thermal processing by short-lived radionuclides that would lead to loss of supervolatiles, and that allowed comet nuclei to incorporate $\sim 3 \mathrm{Myr}$ old material from the inner solar system.
\end{abstract}

Key words. comets: individual: 67P/Churyumov-Gerasimenko - Kuiper belt: general - protoplanetary disks

\section{Introduction}

Studies of giant planet formation (e.g., Pollack et al. 1996; Alibert et al. 2005; Hubickyj et al. 2005; Dodson-Robinson et al. 2008; Weidenschilling 2008) and migration (e.g., Masset \& Snellgrove 2001; Morbidelli \& Crida 2007; Morbidelli et al. 2007; Walsh et al. 2011) in a gas disk show that Jupiter, Saturn, Uranus, and Neptune likely had a compact orbital configuration at heliocentric distances $r_{\mathrm{h}}=5-15 \mathrm{AU}$ at the time of solar nebula dispersal. An exterior primordial disk stretched from $\sim 15 \mathrm{AU}$ to $\sim 30 \mathrm{AU}$ (Gomes et al. 2004), possibly with a much thinner part reaching to $\sim 45 \mathrm{AU}$ (Parker \& Kavelaars 2010; Parker et al. 2011).

The 15-30 AU region was rich in $\mu \mathrm{m}$-sized grains of ice, organics, silicates, sulfides, and metal that coagulated to form cm-sized porous pebbles (e.g., Blum et al. 2006; Ormel et al. 2007; Blum \& Wurm 2008; Zsom et al. 2010). Such pebbles grew to planetesimals through hierarchical agglomeration (e.g., Weidenschilling 1997; Kenyon \& Luu 1998; Windmark et al. 2012a) and/or gravitational collapse of pebble swarms formed by streaming instabilities (e.g., Youdin \& Goodman 2005; Johansen et al. 2007; Wahlberg Jansson \& Johansen 2014).

According to the Nice model (e.g., Tsiganis et al. 2005; Levison et al. 2008), planetesimal-controlled migration of Jupiter and Saturn drove these gas giants into a mutual mean-motion resonance (MMR) that destabilized the outer solar system and scattered Uranus and Neptune into the primordial disk, thereby forming the Edgeworth-Kuiper belt (EKB), the scattered disk (SD), and much of the Oort cloud (OC). This dramatic event is most probably the explanation for the late heavy bombardment (LHB; Gomes et al. 2005) and occurred $400 \mathrm{Myr}$ after the primordial disk formed (Morbidelli et al. 2012; Marchi et al. 2013).

The comets currently residing in the EKB, SD, and OC could have formed in their current state out of smaller cometesimals 
that are ultimately solar nebula grain aggregates. We generically refer to such bodies as "primordial rubble piles" (Weissman 1986), regardless of whether they formed through hierarchical agglomeration or streaming instabilities. Alternatively, comets could be single collision fragments from a larger parent body or a gravitationally bound bunch of such fragments formed in the aftermath of the catastrophic collision that disrupted the parent. We generically refer to such bodies as "collisional rubble piles" (Stern 1988; Farinella \& Davis 1996; Stern \& Weissman 2001).

To understand whether comets are predominantly primordial rubble piles or collisional rubble piles is of paramount importance for our interpretation of observational data from comets. If they are primordial rubble piles, they are certainly extremely old and teach us about the chemical, mineralogical, and physical properties of the solar nebula and allow us to glean insights into the agglomeration processes that transformed the granular protoplanetary disk into a planetary system. If they are collisional rubble piles, some could potentially have formed as recently as $5 \times 10^{5} \mathrm{yr}$ ago in the EKB or SD, based on the typical transfer time to the inner solar system (Duncan \& Levison 1997). If this is the case, they teach us about evolutionary interior processing of trans-Neptunian objects (TNOs) and the physics of collisional disruption and gravitational re-assembly, but they do not necessarily preserve information about the solar nebula, or have only done so in a restricted way. We need to understand what kind of bodies we are studying, and in which manner comet research contributes to solar system science.

The ESA Rosetta mission to comet 67P/ChuryumovGerasimenko (hereafter 67P) offers a unique opportunity to address this important problem. 67P belongs to the Jupiter family (JF), and the population of Jupiter family comets (JFCs), also known as ecliptic comets (Levison \& Duncan 1997), is considered to primarily leak out of the $\mathrm{SD}$, though $\$ 2 \%$ may have come from the EKB (Duncan \& Levison 1997). 67P is therefore a witness to several important events, processes, and environments in the outer solar system, which is the reason for the importance of cometary science, and ultimately, the motivation for launching Rosetta and other spacecraft missions to comets.

We start by summarizing various observational data from Rosetta that provide clues on the origin of $67 \mathrm{P}$ (Sect. 2). In Sect. 3 we discuss the collisional rubble-pile hypothesis, focusing on the thermal and collisional processing expected for parent bodies of various size, and the expected physical properties of collisional rubble piles. They are compared to the chemical, mineralogical, structural, and physical properties of comet nuclei, primarily as revealed by Rosetta observations and the samples returned by Stardust. Our conclusion is that comets do not display any of the characteristics expected for collisional rubble piles. In Sect. 4 we outline a concurrent comet and TNO formation scenario that leads to the formation of comet-sized bodies with low density, high porosity, weak strength, extensive layering, bi-lobed shape; that are rich in supervolatiles like $\mathrm{CO}$ and $\mathrm{CO}_{2}$, may contain amorphous water ice, and did not experience in situ metasomatism and aqueous alteration. We argue that the primordial disk was dynamically cold, and that the survival of small bodies against collisional disruption was high. We call these bodies primordial rubble piles, and we demonstrate in Sect. 5 that their properties match those of comet nuclei. In Sect. 6 we discuss in detail the reasons why our conclusions differ from those of Morbidelli \& Rickman (2015) and Rickman et al. (2015), who argue that rubble-pile formation through catastrophic collisions is an expected outcome of the Nice model. Our summary and conclusions are given in Sect. 7.

\section{Clues about the origin of 67P from Rosetta}

We here summarize the observations of 67P by Rosetta of direct relevance for the origin of the comet, focusing on mass, bulk density, and porosity in Sect. 2.1, tensile, shear, and compressive strength in Sect. 2.2, nucleus morphology in Sect. 2.3, internal mass distribution in Sect. 2.4, spectrophotometric properties in Sect. 2.5, and volatiles in Sect. 2.6.

\subsection{Mass, bulk density, and porosity}

The first convincing evidence that comet nuclei have bulk densities substantially below that of solid water ice was provided by Rickman (1986) using the non-gravitational force (NGF) technique, thereby confirming a previous suggestion by Wallis $\&$ MacPherson (1981). Subsequent NGF modeling of 67P by Rickman et al. (1987) yielded a mass $10^{12} \leq M \leq 1.3 \times 10^{13} \mathrm{~kg}$, while Sosa \& Fernández (2009) obtain $M=1.5( \pm 0.6) \times 10^{13} \mathrm{~kg}$. Davidsson \& Gutiérrez (2005) presented density solutions as a function of spin axis orientation. For orientation "CM", located merely $7.4^{\circ}$ from the actual pole position $(\alpha, \delta)=\left(69.3^{\circ}, 64.1^{\circ}\right)$ (Sierks et al. 2015) in the equatorial system, they obtain $M=$ $1.06 \times 10^{13} \mathrm{~kg}$ with one thermophysical model and $M=1.13 \times$ $10^{13} \mathrm{~kg}$ with another, with $25 \%$ error bars. The mass of $67 \mathrm{P}$ determined by Rosetta/RSI is $9.982( \pm 0.003) \times 10^{12} \mathrm{~kg}$ (Pätzold et al. 2016). This illustrates the accuracy of NGF modeling when the comet spin axis orientation is known.

Because Davidsson \& Gutiérrez (2005) overestimated the volume of $67 \mathrm{P}$ their bulk density estimate $\rho_{\text {bulk }}=$ $330_{-60}^{+90} \mathrm{~kg} \mathrm{~m}^{-3}$ is too low. Accurate determinations of the volume by Rosetta/OSIRIS yield $\rho_{\text {bulk }}=535 \pm 35 \mathrm{~kg} \mathrm{~m}^{-3}$ (Preusker et al. 2015) and $\rho_{\text {bulk }}=532 \pm 7 \mathrm{~kg} \mathrm{~m}^{-3}$ (Jorda et al. 2016) depending on the applied shape model. Errors in the bulk density estimate as high as in Davidsson \& Gutiérrez (2005) are not expected for three other comets studied with the same technique, because spin axis orientations and volumes for these targets were better known at the time of analysis thanks to spacecraft flybys. Therefore, $\rho_{\text {bulk }}=180-300 \mathrm{~kg} \mathrm{~m}^{-3}$ for $19 \mathrm{P} /$ Borrelly (Davidsson \& Gutiérrez 2004), $\rho_{\text {bulk }} \leq 600-800 \mathrm{~kg} \mathrm{~m}^{-3}$ for 81P/Wild 2 (Davidsson \& Gutiérrez 2006), and $\rho_{\text {bulk }}=450 \pm$ $250 \mathrm{~kg} \mathrm{~m}^{-3}$ for 9P/Tempel 1 (Davidsson et al. 2007) may be reliable. For 9P/Tempel 1 Richardson et al. (2007) obtain $\rho_{\text {bulk }}=$ $400_{-200}^{+600} \mathrm{~kg} \mathrm{~m}^{-3}$ by modeling the dynamics of the ejecta plume created during the Deep Impact collision experiment. Thus, the nominal density estimates for 9P/Tempel 1 based on two different techniques are mutually consistent. For the 29 comets studied by Rickman et al. (1987), and the 10 comets studied by Sosa \& Fernández (2009), their individual bulk densities may be less reliable due to unknown spin states and volumes, but the average bulk density of $\rho_{\text {bulk }}<500 \mathrm{~kg} \mathrm{~m}^{-3}$ shows that a low density is typical of JFCs. Additionally, there are no known comet nuclei that spin so fast that a bulk density in excess of $\rho_{\text {bulk }} \approx 600 \mathrm{~kg} \mathrm{~m}^{-3}$ is needed to avoid disruption (Davidsson 2001; Weissman et al. 2004).

Porosity estimation requires a specification of composition. The dust-to-gas mass ratio in the $67 \mathrm{P}$ coma is $4 \pm 2$ (Rotundi et al. 2015 ), indicating an ice mass fraction of $14 \% \leq f_{\text {ice }} \leq 33 \%$. An estimate of the compacted density can also be obtained by starting out with the elemental abundances of CI carbonaceous chondrites as given in Lodders (2003). We assume that all $\mathrm{H}$ atoms are used to form water, requiring half of that number from $\mathrm{O}$. The remaining $\mathrm{O}$ is assumed to combine with all $\mathrm{Mg}$, $\mathrm{Si}, \mathrm{Al}, \mathrm{Ca}$, and $\mathrm{Na}$ to form silicates. We assume that all $\mathrm{C}$ and $\mathrm{N}$ form organics, and that all $\mathrm{Fe}, \mathrm{Ni}$, and $\mathrm{S}$ form metal and 
sulfides. This yields $25 \%$ metal/sulfides, $42 \%$ rock/organics, and $32 \%$ ice by mass (i.e., similar to the estimate above). Averaging the densities of low-sulfur iron $\left(\rho=7100 \mathrm{~kg} \mathrm{~m}^{-3}\right)$ and troilite $\left(\rho=4600 \mathrm{~kg} \mathrm{~m}^{-3}\right)$ for metal/sulfides (Tesfaye Firdu \& Taskinen 2010), averaging the densities of enstatite $\left(\rho_{\mathrm{e}}=3270 \mathrm{~kg} \mathrm{~m}^{-3}\right.$, Horai 1971) and meteoritic graphite $\left(\rho=2100 \mathrm{~kg} \mathrm{~m}^{-3}\right.$, Amari et al. 2012) for rock/organics, and applying $\rho_{\mathrm{i}}=917 \mathrm{~kg} \mathrm{~m}^{-3}$ for ice (Weast 1974) yields a compact density $\rho_{\mathrm{A}}=1820 \mathrm{~kg} \mathrm{~m}^{-3}$ that we call "composition A". The corresponding porosity for $67 \mathrm{P}$ is $\psi_{\mathrm{A}}=(71 \pm 2) \%$. If the ice fraction is increased to $65 \%$ (comets may be less depleted in $\mathrm{H}, \mathrm{O}, \mathrm{C}$, and $\mathrm{N}$ than $\mathrm{CI}$ chondrites) we obtain "composition B" with $\rho_{\mathrm{B}}=1230 \mathrm{~kg} \mathrm{~m}^{-3}$ and $\psi_{\mathrm{B}}=$ $(57 \pm 3) \%$. Greenberg (1998) recommended $\rho=1650 \mathrm{~kg} \mathrm{~m}^{-3}$, roughly the average of $\rho_{\mathrm{A}}$ and $\rho_{\mathrm{B}}$. The CONSERT instrument inferred a porosity of $75 \%-85 \%$ (Kofman et al. 2015), which is consistent with composition A. Hapke modeling of OSIRIS photometric measurements yield a porosity of $87 \%$ for the surface (Fornasier et al. 2015). These values are similar to the $75 \%-$ $88 \%$ porosity for the surface of 9P/Tempel 1 obtained by Ernst $\&$ Schultz (2007) when analyzing the impact flash created during the Deep Impact collision experiment. Evidently, low densities and high porosities may be ubiquitous in comet nuclei.

\subsection{Tensile, shear, and compressive strength}

The strength on small $(0.1-1 \mathrm{~m})$ size scales has been estimated at Agilkia (first touch-down of Philae) in Ma' ${ }^{1}{ }^{1}$ and at Abydos (the final landing site) in Bastet. Agilkia is covered by a $\gtrsim 0.2 \mathrm{~m}$ thick granular layer, potentially overlying a harder substrate, having a compressive strength of $S_{\mathrm{c}} \leq 1-3 \mathrm{kPa}$ (Biele et al. 2015). Groussin et al. (2015) find a preferred range $S_{\mathrm{c}}=30-150 \mathrm{~Pa}$ and an upper limit of $S_{\mathrm{c}}<1.5 \mathrm{kPa}$. Abydos consists of consolidated terrain (Thomas et al. 2015b) with $S_{\mathrm{c}} \approx 2 \mathrm{MPa}$ according to Philae/MUPUS-PEN (Spohn et al. 2015). The higher value is similar to that of sintered porous ice (Spohn et al. 2015) and Biele et al. (2015) suggest that this hard surface is the result of solar processing, and that it is not representative of pristine material.

The strength on large $(10 \mathrm{~m}-1 \mathrm{~km})$ size scales is more representative of the less processed interior. Groussin et al. (2015) derived the tensile strength $S_{\mathrm{t}}$ on 67P by considering collapsed overhangs, and the shear strength $S_{\mathrm{s}}$ by considering boulders on smooth-material slopes. The values are low, $S_{\mathrm{t}}=3-15 \mathrm{~Pa}$ (upper limit $S_{\mathrm{t}}<150 \mathrm{~Pa}$ ) and $S_{\mathrm{s}}=4-30 \mathrm{~Pa}$. To support the Hathor wall $S_{\mathrm{s}}>30 \mathrm{~Pa}$ is needed in that region. For comparison, $100 \mathrm{~kg} \mathrm{~m}^{-3}$ snow has $S_{\mathrm{t}}=100 \mathrm{~Pa}$ (Hagenmuller et al. 2014).

\subsection{Morphological features}

The global shape of $67 \mathrm{P}$ is dominated by two lobes connected by a short neck. The dimensions of the large lobe is $4.10 \times 3.52 \times$ $1.63 \mathrm{~km}$ and the small lobe measures $2.5 \times 2.14 \times 1.64 \mathrm{~km}$ (Jorda et al. 2016). Their volume ratio is 2.4 . Two independent lines of evidence suggest that the two lobes once were separately formed bodies that merged in a low-velocity collision, as opposed to a single body that developed a neck through erosion. First, the thermophysical model of Davidsson \& Rickman (2014) used by Sierks et al. (2015), and that by Keller et al. (2015), consistently show that the energy input in the Hapi valley and Hathor cliff is lower than elsewhere when integrated throughout the orbit while nucleus rotation, shadowing, and self heating are accounted for.

See Fig. 1 in Thomas et al. (2015b) for region names.
Runaway erosion caused by radiation focusing in a cavity is therefore not a viable explanation. Second, the surfaces of both lobes are characterized by thick $(\$ 650 \mathrm{~m})$ layers that envelope the lobes individually (Massironi et al. 2015). Presumably this layering formed during accretion of the lobes, demonstrating that they had qualitatively similar evolutionary histories prior to their merger. Extensive layering is also evident on 9P/Tempel 1 (Thomas et al. 2007) according to Deep Impact images. Bilobed shapes are found for 8P/Tuttle (Harmon et al. 2010), 103P/Hartley 2 (A'Hearn et al. 2011), 19P/Borrelly (Oberst et al. 2004), and possibly 1P/Halley (Merényi et al. 1990; Keller et al. 2004). This suggests that the final phase of comet nucleus formation often is the merger between two similarly-sized cometesimals. However, the single-lobed comets 81P/Wild 2 (Brownlee et al. 2004) and 9P/Tempel 1 (A'Hearn et al. 2005) show that mergers do not necessarily take place. Triple- or multiple-lobed nuclei are yet to be discovered, if they exist. It is an interesting but highly speculative possibility that the Hatmehit basin is what remains of a contact area with a third lobe that was lost in a splitting event. Boehnhardt (2004) lists $\sim 40$ comets that have been observed to split. 67P is not one of those, and we do not suggest a recent event, but a potential tidal split during the time $67 \mathrm{P}$ was a Centaur and may have encountered the giant planets repeatedly.

The merger between similarly-sized cometesimals on the largest size scale leads to the question if similar mergers have taken place on smaller size scales. The layering suggests sweepup of material and that most cometesimals may have flattened on impact (Belton et al. 2007). Erosion of the nucleus by sublimation has been sufficient to create an intricate system of terraces (Massironi et al. 2015), and has weakened the roofs above subsurface cavities to the point where collapse has created large pits (Vincent et al. 2015). Also, sublimation has erased all impact craters but one (Sierks et al. 2015), and airfall has covered large parts of the nucleus (Thomas et al. 2015a). Thus it is not obvious that cometesimals will be visible, even if they exist. Yet, possible evidence of medium-sized cometesimals in 67P are found in the Bastet region. Figure 1 shows three circular features viewed face-on and labeled A, B, and C. Feature B penetrates A slightly, and there is no obvious cavity where the three features meet. If these are merged cometesimals, the collisions were sufficiently strong to remove large-scale void space by deformation, yet weak enough not to completely ruin the structural integrity of the cometesimals.

Figure 2 shows A, B, and C seen from above the small lobe, with the large lobe in the background. Although complicated by an open pit at its margin feature A shows a globally curved shape seen against Hapi, and the same is evident for features $\mathrm{C}$ (seen against the shadowed neck) and B. Fig. 3 shows a third view where the curved silhouette of $\mathrm{C}$ is seen against the sky. A series of long thin shadows, giving the impression of a continuous crack, has been marked with arrows and may show how feature $\mathrm{C}$ is attached to the small lobe. Visual inspection of shape models (Preusker et al. 2015; Jorda et al. 2016) confirms that A, B, and $C$ have shapes resembling spherical caps and we call these structures positive relief features or PRFs.

In numerous locations the surface material of $67 \mathrm{P}$ is cloddy or lumpy on the size scale of a few meters. Examples include the goosebumps discussed by Sierks et al. (2015) shown magnified in Fig. 4. The diameter of 145 goosebumps ranges $1.2 \mathrm{~m}-3.4 \mathrm{~m}$ with an average of $2.2 \mathrm{~m}$. Another example is found in Imhotep, with Fig. 5 showing context, while Fig. 6 is a close-up. These clods are somewhat larger $(3.1 \mathrm{~m}$ mean diameter for 80 clods) than the Seth goosebumps, tend to have a larger variability in 


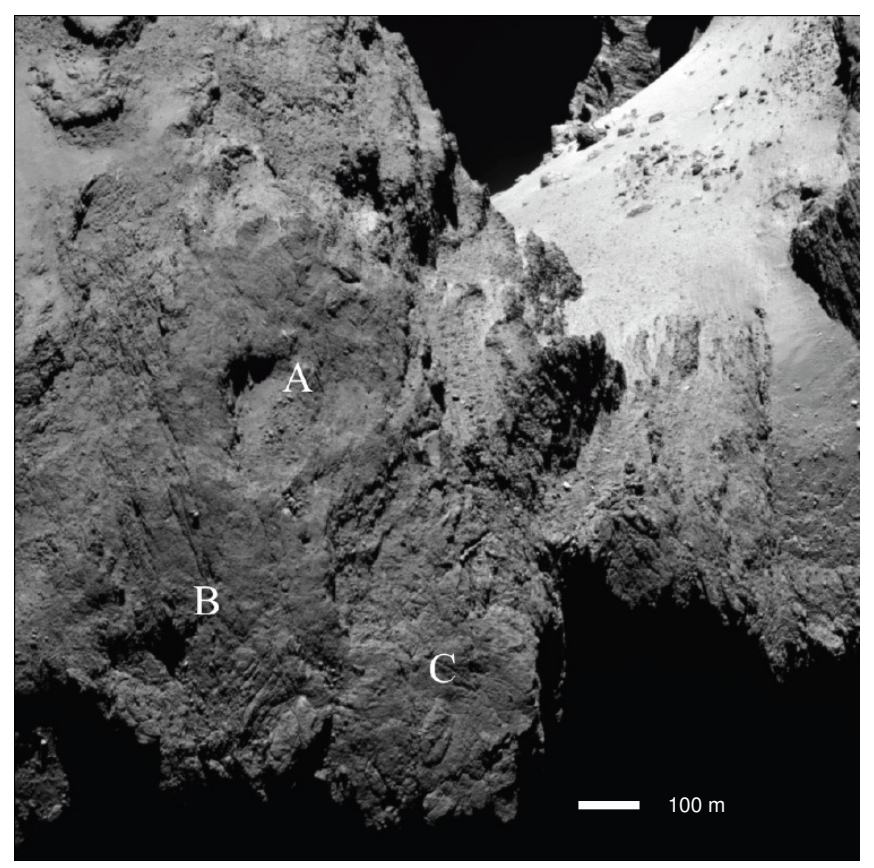

Fig. 1. Face-on view of three PRFs in the Bastet region on the small lobe from a distance of $69.9 \mathrm{~km}$. They may be accreted cometesimals. Feature A has diameter $400 \mathrm{~m}$ and B $450 \mathrm{~m}$. Feature C is more elongated and measures $320 \times 480 \mathrm{~m}$. The Hapi valley is seen in the background. Sequence STP016_SHAP4S, image NAC_2014-0903T06.44.22.578Z_ID00_1397549400_F22.img.

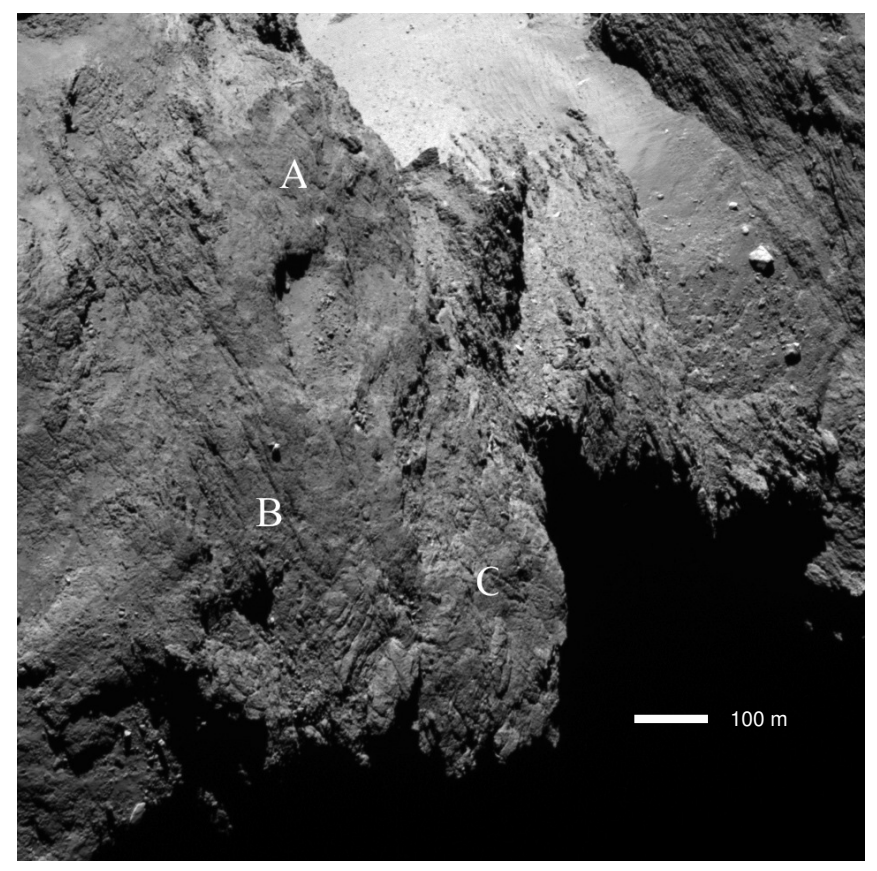

Fig. 2. View from above of three PRFs in the Bastet region from a distance of $59.9 \mathrm{~km}$. Sequence STP016_SHAP4S, image NAC_2014-0902T18.44.24.557Z_ID00_1397549200_F22.img.

terms of size ( $1.8 \mathrm{~m}-5.6 \mathrm{~m}$ range $)$ and appear to be somewhat more irregular.

Similar features are seen in the Anubis and Atum regions (Fig. 7 for context). Figure 8 shows clods in Anubis that are very similar to those in Imhotep both in terms of appearance and size ( $2.5 \mathrm{~m}$ mean diameter for $151 \mathrm{clods}$, range $1.4 \mathrm{~m}-4.7 \mathrm{~m}$ ). These clods appear rather unconsolidated. The southern parts of the

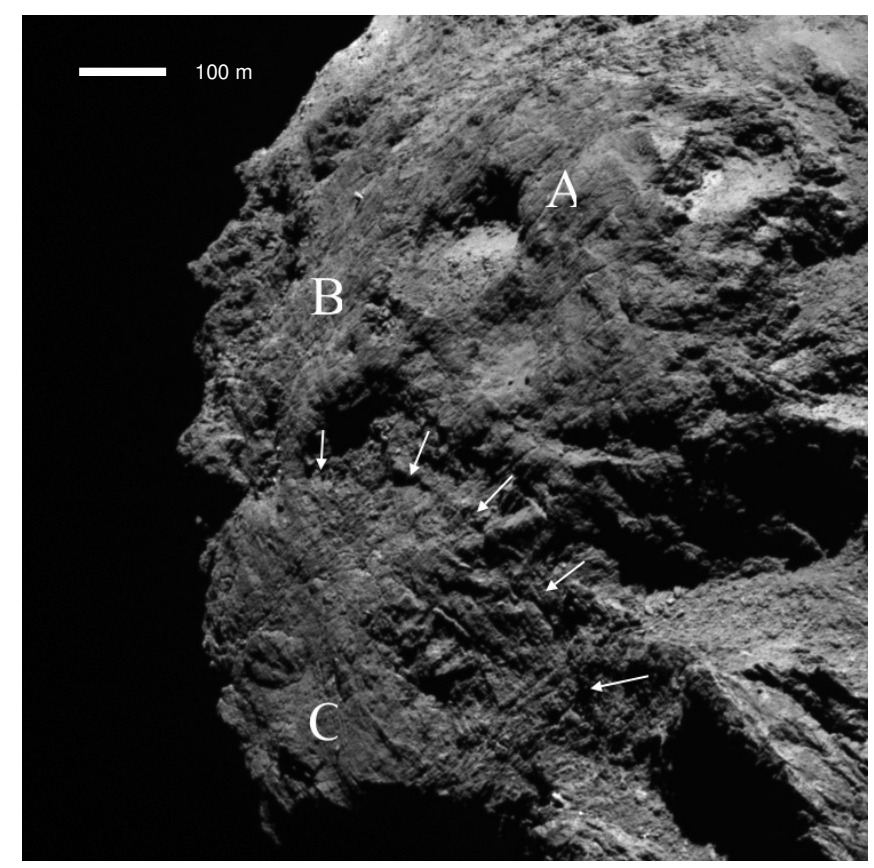

Fig. 3. View from below (as if standing on the large lobe looking toward the small lobe) of three PRFs in the Bastet region from a distance of $65.6 \mathrm{~km}$. Sequence STP015_SHAP4S_002, image NAC_201408-27T01.41.54.606Z_ID00_1397549000_F22.img.
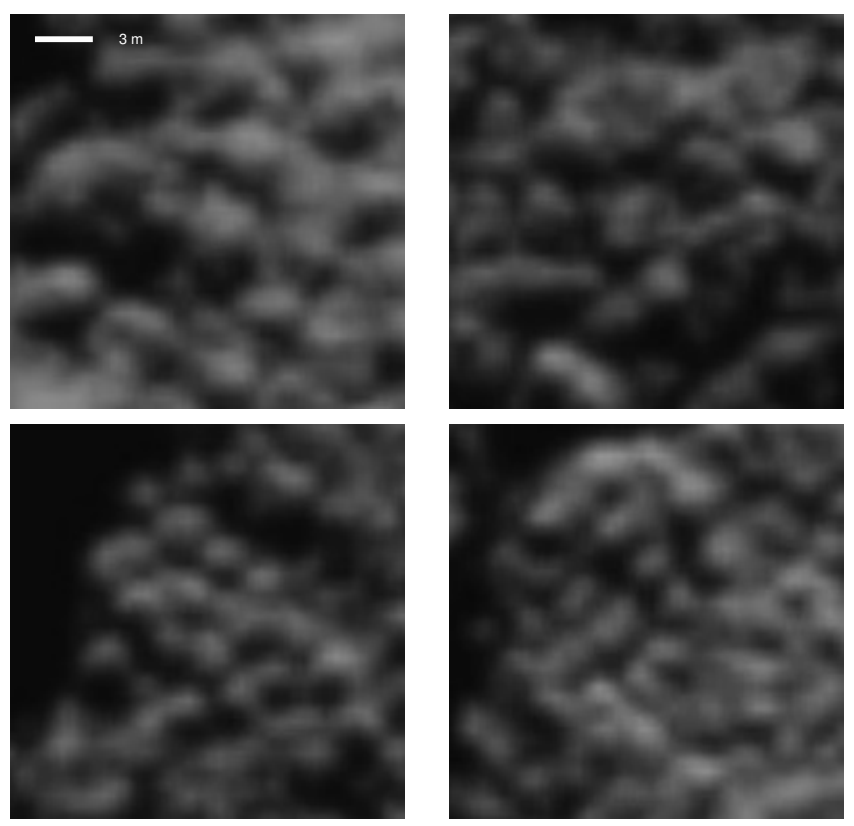

Fig. 4. Close-up of goosebumps located on the walls of a large pit in the Seth region (for context, see Fig. 8a in Sierks et al. 2015) from a distance of $26.9 \mathrm{~km}$. Sequence STP018_SHAP5b, image NAC_201409-22T00.50.19.311Z_ID00_1397549200_F22.img.

Atum region (Fig. 9) shows what seem to be consolidated clods (diameters mostly in the $3.2-4.6 \mathrm{~m}$ range) in the lower half of the image. Their convex surfaces and their altitude variability distinguish them from structures formed by polygonal cracking in smooth consolidated material (El-Maarry et al. 2015b). Toward the upper right of Fig. 9 the clods decrease in diameter to 1.4$2.2 \mathrm{~m}$ and look similar to the goosebumps in Seth. For 214 clods in the entire region the mean diameter is $2.4 \mathrm{~m}$ and the range is $1.4 \mathrm{~m}-4.6 \mathrm{~m}$. There are at least three mechanisms that may have 


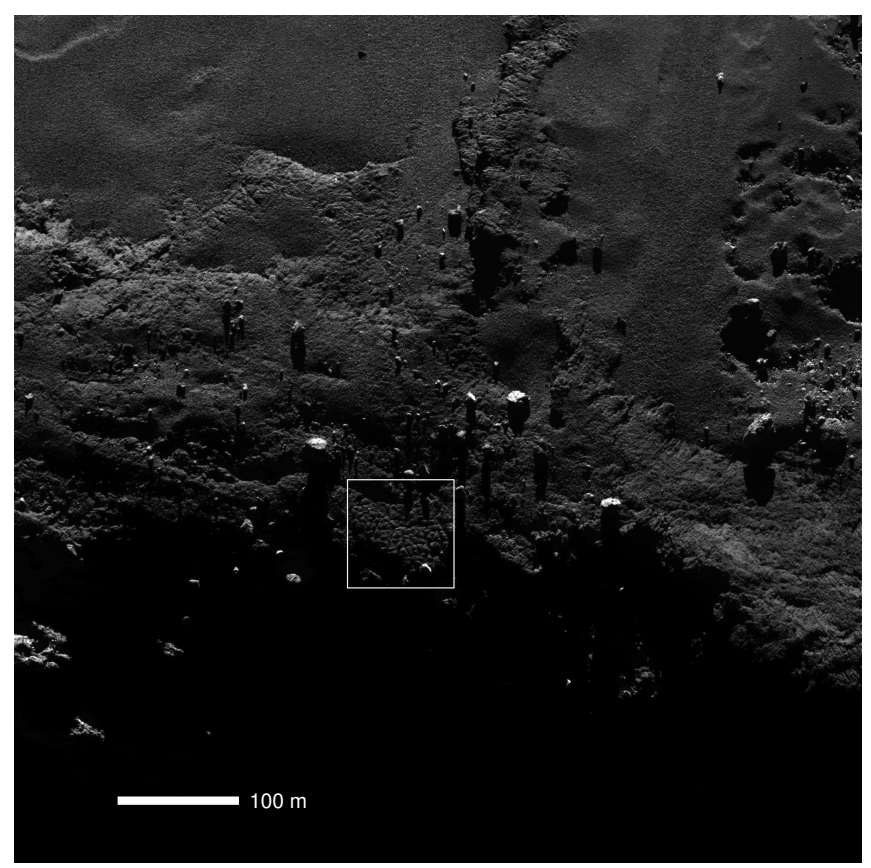

Fig. 5. Context image of clods in the southern Imhotep region from a distance of $17.0 \mathrm{~km}$. The white square contains clods shown in Fig. 6. Sequence STP021_SHAP6b_NAC22, image NAC_2014-1006T18.53.19.544Z_ID00_1397549100_F22.img.

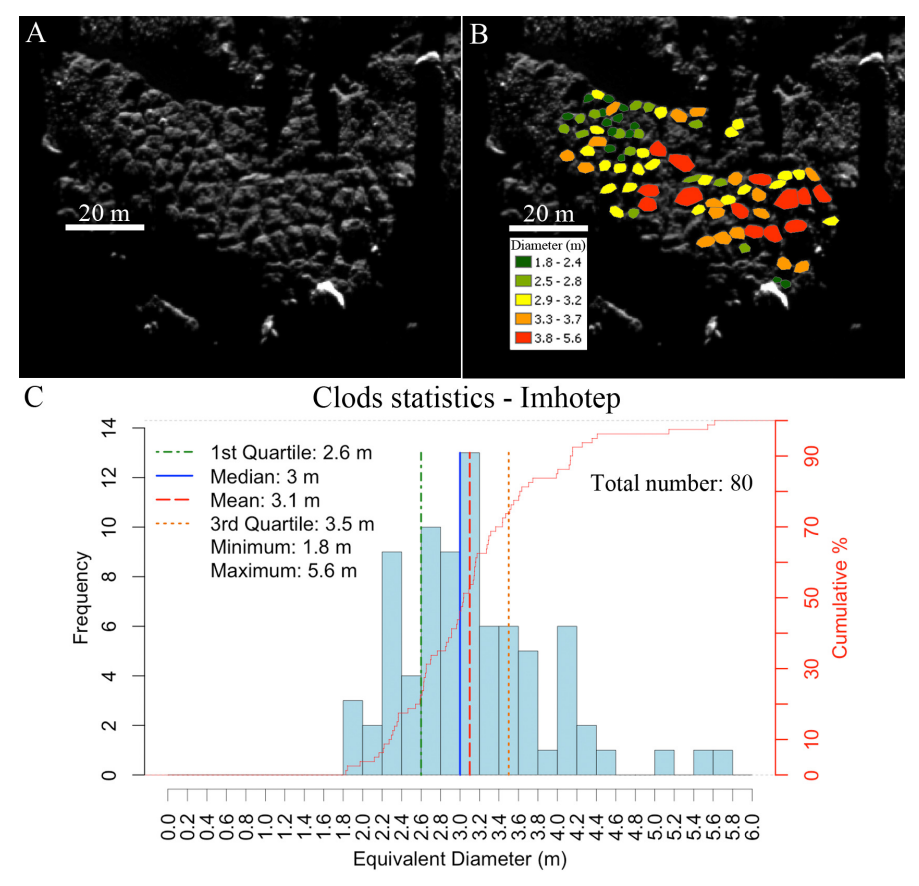

Fig. 6. Close-up of clods in the southern Imhotep region from a distance of $17.0 \mathrm{~km}$. See Fig. 5 for context. Sequence STP021_SHAP6b_NAC22, image NAC_2014-1006T18.53.19.544Z_ID00_1397549100_F22.img.

formed these goosebumps and clods; (1) fracturing in homogeneous granular media because of thermal gradients (El-Maarry et al. 2015a; Auger et al. 2016); (2) they form by the previously mentioned fracturing process, being regulated by an intrinsic lumpiness of the medium; (3) no fracturing is involved and an intrinsic lumpiness is made visible during sublimation. If any of the last two scenarios is correct, goosebumps and clods

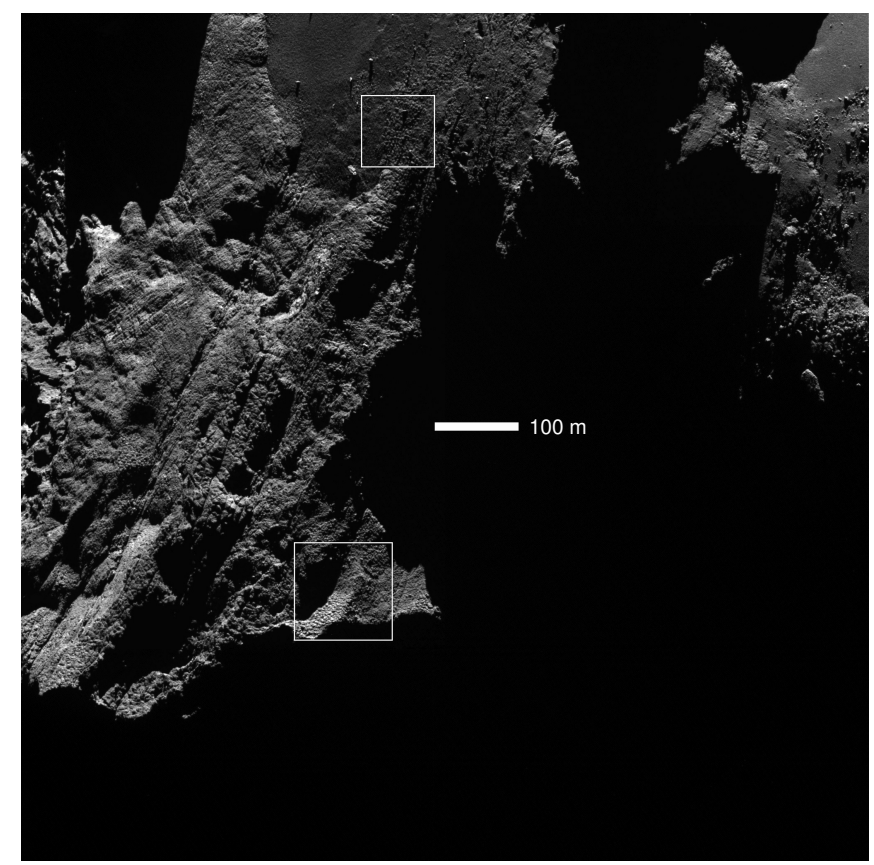

Fig. 7. Context image of the Atum (consolidated) and Anubis (smooth, upper center) regions from a distance of $24.6 \mathrm{~km}$. The upper square shows the location of Fig. 8, and the lower that of Fig. 9. Sequence STP032_LIMB_SCAN_001, image NAC_2014-1204T01.41.55.426Z_ID00_1397549400_F22.img.

suggest that cometesimals with diameters $2.5 \pm 1 \mathrm{~m}$ may have been particularly numerous and resilient.

The Philae/ROLIS camera imaged a $\sim 5 \mathrm{~m}$ boulder at $7.1 \pm$ $0.1 \mathrm{~cm} \mathrm{px}^{-1}$ resolution that was covered by $\mathrm{dm}$-sized rounded knobs (Mottola et al. 2015). These authors speculate that the boulder possibly is a primordial conglomerate of smaller units. The sunlit wall imaged at $\sim 1 \mathrm{~mm} \mathrm{px}^{-1}$ resolution by the Philae/CIVA camera at Abydos is partially an agglomerate consisting of structures in the mm-cm range (Bibring et al. 2015). These high-resolution observations suggest that m-sized features are not necessarily homogeneous assemblages of $\mu \mathrm{m}$-sized grains, but may contain substructure on the $\mathrm{mm}$ to dm-scale.

\subsection{Internal mass distribution}

The Rosetta/Philae/CONSERT experiment (Kofman et al. 2015) probed a 560-760 $\mathrm{m}$ path through the evening sector (roughly at Maftet and Serqet) and a 190-710 m path through the morning sector (roughly at Bastet) on the small lobe. Kofman et al. (2015) interpret the absence of volume scattering as the medium being homogeneous on the size scale of a few times the $\lambda=3.3 \mathrm{~m}$ wavelength. Yet, the detection of 2-3 well-defined propagation paths show the potential presence of large $(\gg 10 \mathrm{~m})$ structures within the small lobe (Kofman et al. 2015). Further analysis of the same data by Ciarletti et al. (2015) shows that the dielectric constant decreases by $\sim 40 \%$ with depth within $\sim 150 \mathrm{~m}$ of the surface. This is interpreted by Ciarletti et al. (2015) as a $\sim 15 \%$ increase in porosity over that depth, or that the dust-to-ice mass ratio drops from $\sim 4$ to $\sim 0.1$. In either case, the density near the surface is higher than $\sim 150 \mathrm{~m}$ below.

Further evidence of a heterogeneous mass distribution is a measured discrepancy between the center of figure and the center of mass (if calculated for a uniform interior) that amounts to $\{18 \pm 7,-32 \pm 4,16 \pm 10\} \mathrm{m}$, as well as a $4.0^{\circ} \pm 1.9^{\circ}$ tilt of 


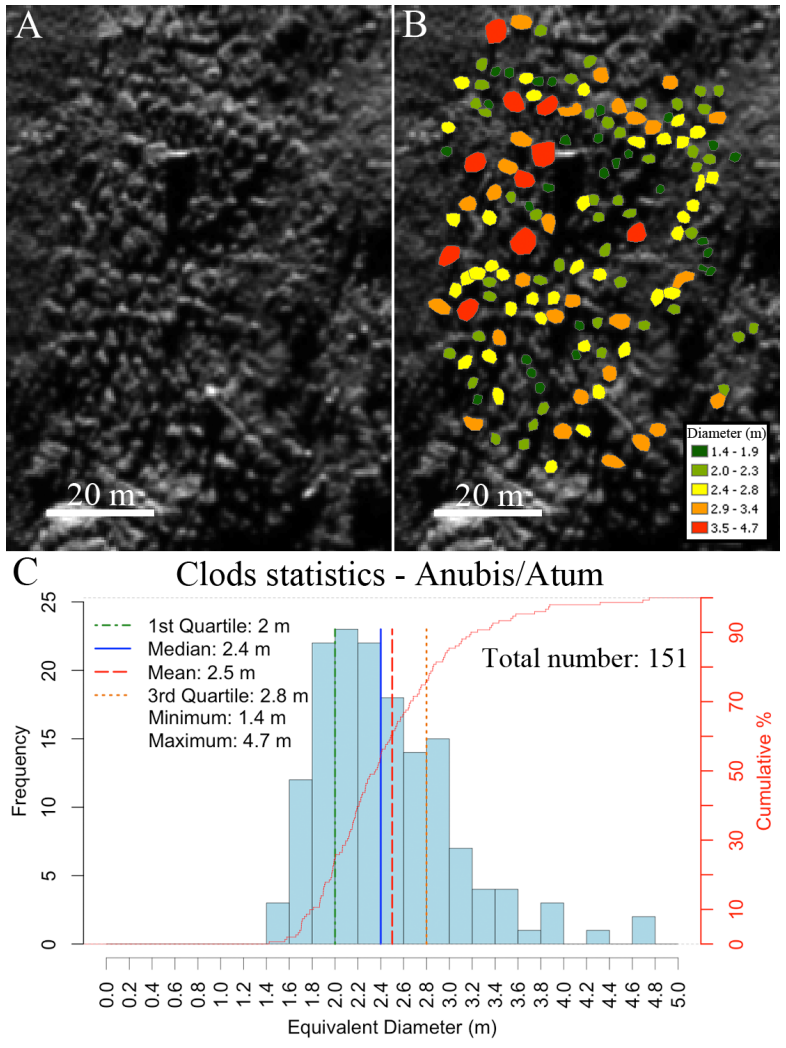

Fig. 8. Close-up of clods on the boundary between the Anubis and Atum regions from a distance of $24.6 \mathrm{~km}$. See Fig. 7 for context. Sequence STP032_LIMB_SCAN_001, image NAC_2014-1204T01.41.55.426Z_ID00_1397549400_F22.img.

the nucleus $z$-axis with respect to the corresponding principal axis (Jorda et al. 2016). This is consistent with the large lobe having a $5-15 \%$ higher density than the small lobe, although some level of internal density variation is necessary to achieve an exact match. Jorda et al. (2016) find that a uniform mass distribution implies normalized principal moments of inertia of $I_{x}: I_{y}: I_{z}=1: 1.85( \pm 0.04): 1.99( \pm 0.04)$, thus $I_{z}-I_{y}=0.14 \pm 0.04$.

Rosetta/RSI measured the gravity field up to degree and order 2, and Pätzold et al. (2016) conclude that the nucleus is homogeneous, has constant density on a global scale, and lacks large voids. However, Pätzold et al. (2016) do not discuss the implications of their $C_{11}, S_{11}, C_{21}$, and $S_{21}$ values and error bars not overlapping those of the Preusker et al. (2015) and Jorda et al. (2016) shape models with constant density, for example, whether these discrepancies are consistent with the heterogeneity discussed by Ciarletti et al. (2015). Also, they do not quantify the size of voids they can exclude or the upper limit on global density variations.

Gutiérrez et al. (2016) analyzed the $\sim 270 \mathrm{~h}$ periodicity measured for the changing spin axis orientation. Focusing on the orbital segment for which the rotation period $P=12.4$ h (Mottola et al. 2014) remained fixed, they find that torque-free rotation requires a precession period $P_{\phi}=6.35 \mathrm{~h}$, spin period $P_{\psi}=$ $13 \mathrm{~h}$, and nutation period $P_{\theta}=6.5 \mathrm{~h}$ to explain the observed $P_{\mathrm{c}}=\left(P_{\phi}^{-1}-P_{\theta}^{-1}\right)^{-1} \approx 270 \mathrm{~h}$ variability. This is only possible if $I_{z}=0.963+0.988 I_{y}$ (Gutiérrez et al. 2016). The resulting $I_{z}-I_{y} \approx 0.96$ is larger than for a homogeneous mass distribution, suggesting that mass may be concentrated to the equatorial plane in general and to the surface in particular.
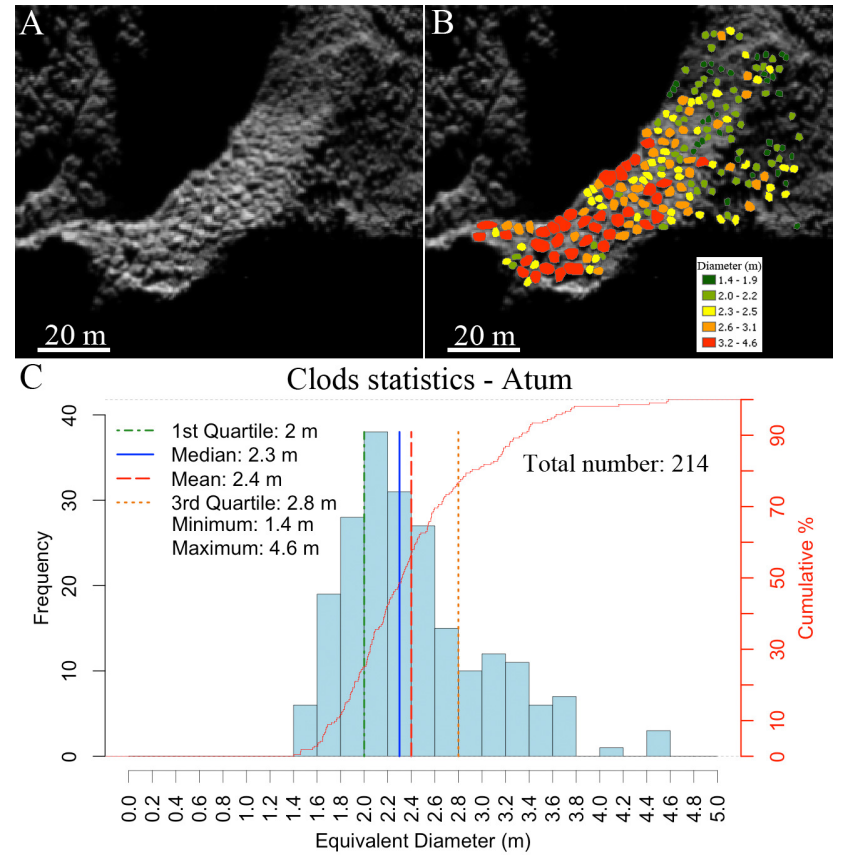

Fig. 9. Close-up of clods in the Atum region from a distance of $24.6 \mathrm{~km}$. See Fig. 7 for context. Sequence STP032_LIMB_SCAN_001, image NAC_2014-12-04T01.41.55.426Z_ID00_1397549400_F22.img.

\subsection{Spectrophotometry}

The visual and near-infrared reflectance spectrum of $67 \mathrm{P}$ has been explored with Rosetta/OSIRIS broadband photometry in the $0.25-1.0 \mu \mathrm{m}$ wavelength region (Fornasier et al. 2015), and Rosetta/VIRTIS spectroscopy in the $0.5-4.0 \mu \mathrm{m}$ region (Capaccioni et al. 2015). The observations indicate potential absorption at $0.29 \mu \mathrm{m}$, possibly due to $\mathrm{SO}_{2}$ ice (Fornasier et al. 2015). A broad asymmetric absorption band in the $2.9-3.6 \mu \mathrm{m}$ region is evident, centered at $3.2-3.3 \mu \mathrm{m}$. This feature is likely due to the $\mathrm{C}-\mathrm{H}$ group in aliphatic and aromatic hydrocarbons, possibly in combination with the $\mathrm{O}-\mathrm{H}$ group in carboxylic acids (Capaccioni et al. 2015). In the Hapi region the short-wavelength edge of the absorption feature shifts to $2.8 \mu \mathrm{m}$ (Capaccioni et al. 2015) while the spectral slope becomes less red and the albedo increases (Fornasier et al. 2015), suggesting weak $3.0 \mu \mathrm{m}$ water ice absorption.

It is equally interesting to note what is not seen in the photometry and spectra. Laboratory spectra of aqueously altered CI, $\mathrm{CM}$, and CR carbonaceous chondrites do not provide a good match to 67P (Capaccioni et al. 2015). For the vast majority of the nucleus surface there is no detectable absorption at $0.70 \pm 0.12 \mu \mathrm{m}$ due to $\mathrm{Fe}^{2+} \rightarrow \mathrm{Fe}^{3+}$ charge transfer in oxidized iron. That means that phyllosilicates are rare or absent, hence there has been little to no aqueous alteration (e.g., Rivkin et al. 2002). Possible exceptions are the meter-sized bright and spectrally atypical boulders described by Pommerol et al. (2015). A few of these appear to have a weak $0.70 \mu \mathrm{m}$ absorption feature according to Oklay et al. (2016). These authors propose that the unusual boulders may be foreign material that was swept up by the comet nucleus.

Phyllosilicates also have absorption features at $3.0 \pm 0.4 \mu \mathrm{m}$ due to structural hydroxyl $(\mathrm{OH})$ and interlayer or absorbed $\mathrm{H}_{2} \mathrm{O}$ (e.g., Rivkin et al. 2002). Meteoritic hydrated minerals do not suffer significant loss of water unless heated above $\sim 650 \mathrm{~K}$ (Garenne et al. 2014), therefore solar heating is not capable of dehydrating phyllosilicates. The lack of a $3.0 \mu \mathrm{m}$ absorption 
feature in 67P spectra (except in places where regional context, albedo, and spectral slope indicates the presence of water ice) provides further evidence that 67P did not experience in situ aqueous alteration.

Lisse et al. (2006, 2007) propose the presence of the phyllosilicate nontronite in mid-IR Spitzer spectra of comets 9P/Tempel 1 and C/1995 O1 (hereafter Hale-Bopp). It is the main host of sodium in their spectral fit. Nontronite is common on planet Mars and was identified in Mars Express/OMEGA spectra from absorption features at $1.41 \mu \mathrm{m}$, $1.9 \mu \mathrm{m}$ and $2.29 \mu \mathrm{m}$ (Poulet et al. 2005). The Rosetta/COSIMA analyzer collected Na-rich grains in the 67P coma (Schulz et al. 2015), suggesting that grains of this type are common on the nucleus surface. The absence of near-IR absorption at these wavelengths in Rosetta/VIRTIS spectra (Fig. 1 in Capaccioni et al. 2015 ) shows that the Na-bearing host on 67P is not likely nontronite.

\subsection{Volatiles}

Rosetta/OSIRIS observations show that the dust jet intensity correlates with illumination conditions at the source regions (Vincent et al. 2016). This diurnal variation suggests that the sublimating water ice that drives the jet activity is located within a few skin depths of the surface. The skin depth (Spencer et al. 1989) is given by

$$
L=\frac{\Gamma}{\left(c_{\mathrm{i}} \rho_{\mathrm{i}} f_{\text {ice }}+c_{\mathrm{e}} \rho_{\mathrm{e}}\left(1-f_{\text {ice }}\right)\right)(1-\psi)} \sqrt{\frac{P}{2 \pi}},
$$

where the thermal inertia ${ }^{2}$ is $10 \leq \Gamma \leq 50 \mathrm{MKS}$ for $67 \mathrm{P}$ according to Rosetta/MIRO (Gulkis et al. 2015), and $P=12.4 \mathrm{~h}$ is the rotational period (Mottola et al. 2014). If we use $\Gamma=30 \mathrm{MKS}$, $\psi=0.8, f_{\text {ice }}=0.5 ; \rho_{\mathrm{i}}$ and $\rho_{\mathrm{e}}$ from Sect. 2.1 ; specific heat capacities $c_{\mathrm{i}}=1600 \mathrm{~J} \mathrm{~kg}^{-1} \mathrm{~K}^{-1}$ (Klinger 1981) and $c_{\mathrm{e}}=830 \mathrm{~J} \mathrm{~kg}^{-1} \mathrm{~K}^{-1}$ (Robie et al. 1982) we obtain $L=6 \mathrm{~mm}$. Shi et al. (2016) show that the gradual disappearance of jets, as their footprints rotate into the nightside, is consistent with these $\Gamma$ and $L$ values. Thus the nucleus interior is well-isolated and water ice is present close to the surface.

Rosetta/ROSINA observations show that $\mathrm{H}_{2} \mathrm{O}, \mathrm{CO}$ and $\mathrm{CO}_{2}$ are the three dominant coma gas species in $67 \mathrm{P}$, with average abundance ratios of $\mathrm{CO} / \mathrm{H}_{2} \mathrm{O}=0.13 \pm 0.07$ and $\mathrm{CO}_{2} / \mathrm{H}_{2} \mathrm{O}=$ $0.08 \pm 0.05$ by number (Hässig et al. 2015), thus $\mathrm{CO} / \mathrm{CO}_{2} \approx 1.6$. Surprisingly, molecular oxygen $\mathrm{O}_{2}$ is the fourth most common coma species with $\mathrm{O}_{2} / \mathrm{H}_{2} \mathrm{O}=0.0380 \pm 0.0085$ (Bieler et al. 2015). Furthermore, Rosetta/ROSINA has detected molecular nitrogen with an abundance $\mathrm{N}_{2} / \mathrm{CO}=(5.70 \pm 0.66) \times 10^{-3}$ (Rubin et al. 2015), and argon with an abundance ${ }^{36} \mathrm{Ar} / \mathrm{H}_{2} \mathrm{O}=$ $(0.1-2.3) \times 10^{-5}$ (Balsiger et al. 2015).

The sublimation temperatures of $\mathrm{CO}, \mathrm{O}_{2}, \mathrm{~N}_{2}$, and Ar ices are all in the range $22 \lesssim T_{\mathrm{s}} \lesssim 25 \mathrm{~K}$ (Yamamoto 1985; BarNun et al. 1987; Meech \& Svoreň 2004). These supervolatiles place important constraints on the structure of cometary ice and the thermal history of 67P. Because these species do not form clathrate-hydrates (Notesco \& Bar-Nun 2000) they may partially be trapped in amorphous water ice, or if all water ice was crystalline at the birth of 67P, they must exist in condensed form. We first discuss the case of amorphous water ice.

The release of occluded gas during heating of amorphous water ice is very complex. Laboratory experiments (Bar-Nun et al. 1987; Notesco \& Bar-Nun 2000) show low-level release caused by slow annealing at $80 \mathrm{~K}$, and if $\mathrm{CH}_{3} \mathrm{OH}$ is

\footnotetext{
$21 \mathrm{MKS}=1 \mathrm{~J} \mathrm{~m}^{-2} \mathrm{~K}^{-1} \mathrm{~s}^{-1 / 2}$.
}

present a large portion of the occluded gases are released around $120 \mathrm{~K}$ during formation of methanol clathrate-hydrates. In case methanol is missing, major release is delayed until crystallization into cubic water ice takes place near $137 \mathrm{~K}$. Remaining foreign molecules are released during the transformation from cubic to hexagonal water ice at $\sim 160 \mathrm{~K}$, and then during water ice sublimation at $\sim 180 \mathrm{~K}$. Bar-Nun et al. (1987), Notesco \& Bar-Nun (2000) show that the relative importance of release at $\sim 160 \mathrm{~K}$ and at $\sim 180 \mathrm{~K}$ depends strongly on the amount of occluded gas - the higher the concentration the smaller the fraction released during sublimation. They also find that the total release at $\gtrsim 160 \mathrm{~K}$ may be as large as during crystallization. The correlated release of $\mathrm{O}_{2}\left(T_{\mathrm{s}}=24 \mathrm{~K}\right), \mathrm{CH}_{3} \mathrm{OH}\left(T_{\mathrm{s}}=99 \mathrm{~K}\right)$, and $\mathrm{HCN}\left(T_{\mathrm{s}}=95 \mathrm{~K}\right)$ with water vapor in $67 \mathrm{P}$ measured by Rosetta/ROSINA (Luspay-Kuti et al. 2015) is a potential example of such last-stage release of trapped low-abundance molecules. The release patterns of $\mathrm{CO}\left(T_{\mathrm{s}}=25 \mathrm{~K}\right), \mathrm{CO}_{2}\left(T_{\mathrm{s}}=\right.$ $80 \mathrm{~K})$ and $\mathrm{C}_{2} \mathrm{H}_{6}\left(T_{\mathrm{s}}=44 \mathrm{~K}\right)$ are mutually similar, but differ from that of $\mathrm{H}_{2} \mathrm{O}$ (Luspay-Kuti et al. 2015). This could indicate that these high-abundance supervolatiles primarily are released when water ice crystallizes at $137 \mathrm{~K}$ and when it becomes hexagonal at $\sim 160 \mathrm{~K}$, so that little is left when the water ice starts to sublimate. There is a hint of nonlinearity in the emission of argon and $\mathrm{N}_{2}$ relative to $\mathrm{CO}$ (Rubin et al. 2015; Balsiger et al. 2015), and $\mathrm{CH}_{4}\left(T_{\mathrm{s}}=31 \mathrm{~K}\right)$ does not follow neither $\mathrm{H}_{2} \mathrm{O}$ nor $\mathrm{CO}_{2}$ (LuspayKuti et al. 2015). If amorphous water ice is abundant on 67P, it is unlikely that this comet experienced global heating (e.g., due to short-lived radionuclides) above $\sim 90 \mathrm{~K}$ because that leads to crystallization in just $\sim 10^{5} \mathrm{yr}$ (Schmitt et al. 1989) and massive loss of $\mathrm{CO}$ and $\mathrm{CO}_{2}$.

If $67 \mathrm{P}$ did not contain amorphous water ice at birth, the supervolatiles must exist as frozen ice. Bar-Nun et al. (1987) show that most Ar sublimates at $T_{\mathrm{s}} \approx 23 \mathrm{~K}$ but that a monolayer of $\mathrm{Ar}$ in direct contact with $\mathrm{H}_{2} \mathrm{O}$ remains as a result of a higher surface binding energy. In this case Ar does not sublimate unless $T_{\mathrm{s}} \gtrsim 44 \mathrm{~K}$. If this applies to other supervolatiles, the high abundances of $\mathrm{CO}$ and $\mathrm{O}_{2}$ in 67P suggest that the global temperature never has exceeded $\sim 40 \mathrm{~K}$.

\section{The collisional rubble-pile hypothesis}

To determine if $67 \mathrm{P}$ is composed of fragments of a larger parent body it is necessary to understand formation conditions and processing mechanisms of such parents, and compare with the observed properties of 67P. We first discuss if ${ }^{26} \mathrm{Al}$ should be expected in the early outer solar system, based on our current understanding of the origin of short-lived radionuclides and radial mixing in protoplanetary disks (Sect. 3.1). The consequences in terms of thermal evolution if primordial disk inhabitants contained ${ }^{26} \mathrm{Al}$ are discussed in Sect. 3.2. We then describe observational evidence of ${ }^{26} \mathrm{Al}$-driven processing in the outer solar system, focusing on density and shape in Sect. 3.3 and aqueous alteration in Sect. 3.4. Collisional processing is discussed in Sect. 3.5 and radiometric dating of comet material in Sect. 3.6. With this background, we discuss if $67 \mathrm{P}$ could be a collisional rubble pile originating from a larger parent body (Sect. 3.7).

\subsection{Radioactive aluminum-26 in the outer solar system}

Observations of $1.809 \mathrm{MeV} \gamma$-ray emission by CGRO and INTEGRAL (Diehl et al. 1995; Bouchet et al. 2015) reveal the presence of galactic ${ }^{26} \mathrm{Al}$ with a current interstellar medium steady-state average abundance of ${ }^{26} \mathrm{Al} /{ }^{27} \mathrm{Al}=8.4 \times 10^{-6}$ 
(Diehl et al. 2006). This is a factor of 6-7 below the canonical ${ }^{26} \mathrm{Al}$ abundance at the time of formation of most calciumaluminum-rich inclusions or CAI (MacPherson et al. 1995) in the solar system. Furthermore, significant decay of galactic ${ }^{26} \mathrm{Al}$ may take place during the 0.3-1.6 Myr lifetime (Lee \& Myers 1999; Visser et al. 2002) of the starless core stage that predates collapse into a Class 0 protostar because the half-life of ${ }^{26} \mathrm{Al}$ is just $0.705 \pm 0.024 \mathrm{Myr}$ (Norris et al. 1983). Thus the solar system may have been born with little to no short-lived radionuclides. In fact, near-absence of ${ }^{26} \mathrm{Al}$ and ${ }^{41} \mathrm{Ca}$ during the Class 0 stage may be required to explain the properties of FUN CAI (CAI with fractionation and unidentified nuclear isotope effects), if these formed earlier than other CAI as suggested by Sahijpal \& Goswami (1998) and Thrane et al. (2008). The isotopic anomalies in ${ }^{42} \mathrm{Ca},{ }^{48} \mathrm{Ca}$ (Lee et al. 1978), ${ }^{135} \mathrm{Ba},{ }^{137} \mathrm{Ba},{ }^{145} \mathrm{Nd},{ }^{146} \mathrm{Nd}$ (McCulloch \& Wasserburg 1978), and ${ }^{50} \mathrm{Ti}$ (Clayton et al. 1988) that define FUN CAI besides their lack of ${ }^{26} \mathrm{Al}$ and ${ }^{41} \mathrm{Ca}$, can be interpreted as FUN CAI formation taking place before the protoplanetary disk grew sufficiently large to become well-mixed. Thus, the overwhelming evidence of the high canonical ${ }^{26} \mathrm{Al}$ abundance, from 1500 studies of components from 60 different meteorites (MacPherson et al. 1995), may require local production and/or injection of short-lived radionuclides from the outside during the early Class I stage.

Local production of ${ }^{26} \mathrm{Al}$ by spallation near the protosun would potentially limit its presence at large heliocentric distances. The measured excess of ${ }^{10} \mathrm{~B}$ and possibly ${ }^{7} \mathrm{Li}$ in meteorites (e.g., Chaussidon et al. 2006), being the decay products of ${ }^{10} \mathrm{Be}$ and ${ }^{7} \mathrm{Be}$, is normally interpreted as evidence that spallation indeed took place (e.g., Halliday \& Kleine 2006; Ouellette et al. 2009) because these beryllium isotopes are not products of stellar nucleosynthesis and have short half-lives (1.5 Myr and 53 days). However, the excess of ${ }^{26} \mathrm{Mg}$ (the decay product of ${ }^{26} \mathrm{Al}$ ) does not correlate with the ${ }^{10} \mathrm{~B}$ excess (in fact, ${ }^{10} \mathrm{Be}$ was present in ${ }^{26} \mathrm{Al}$-free FUN CAI, Wood 2004), which rules out spallation as the dominant ${ }^{26} \mathrm{Al}$ producer (Marhas et al. 2002). Furthermore, spallation leads to the wrong ${ }^{41} \mathrm{Ca} /{ }^{26} \mathrm{Al}$ ratio and is incapable of producing ${ }^{60} \mathrm{Fe}$ (Wood 2004) that undoubtedly was present (Shukolyukov \& Lugmair 1993).

Thus, injection of ${ }^{26} \mathrm{Al}$ is perhaps a more attractive scenario, particularly since a $\sim 21 M_{\odot}$ Type II supernova (SNII) produces the major short-lived radionuclide isotopes in proportions similar to those measured in meteorites (Ouellette et al. 2009). Injection during the solar nebula stage (i.e., protostar Class II, equivalently, a classical T Tauri star) may not be optimal because the small radius of the disk $(\sim 30-45 \mathrm{AU})$ requires a nearby $(\sim 0.3 \mathrm{pc})$ SNII to provide sufficient ${ }^{26} \mathrm{Al}$, that potentially would disintegrate the solar nebula (Gounelle \& Meibom 2008). Furthermore, the CAI-forming epoch could have been as short as $2 \times 10^{4}-10^{5} \mathrm{yr}$ according to radiometric dating (Bizzarro et al. 2004; Thrane et al. 2006; Krot et al. 2008), presumably because sufficiently high temperatures for CAI formation only were met during the Class 0 and early Class I stages. These stages only last for $\sim 10^{4} \mathrm{yr}$ and $\sim 10^{5} \mathrm{yr}$ (André \& Montmerle 1994). It is unlikely that the SNII went off exactly during this brief period, to allow for the formation of both FUN CAI and regular CAI. An alternative scenario (Boss \& Vanhala 2000; Vanhala \& Boss 2002; Boss et al. 2008) is suggesting that the SNII exploded during the starless core phase when the large collection area (radius $\sim 5000 \mathrm{AU}$ ) allowed for a more comfortable SNII distance of $\sim 10$ pc. Boss et al. (2008) show that the accreted SNII gas triggers a shock wave that reaches the core center and causes an inside-out collapse that allows the protosun and a small disk (Class 0 ) to form about $\sim 0.1 \mathrm{Myr}$ after contact, before being reached by Rayleigh-Taylor fingers carrying ${ }^{26} \mathrm{Al}$ (early Class I), that have overtaken slower indigenous gas that will reach the disk over the next few $0.1 \mathrm{Myr}$. This scenario is attractive since it relaxes the timing problem of the SNII explosion, and may allow for a brief period of FUN CAI formation in the absence of ${ }^{26} \mathrm{Al}$ and ${ }^{41} \mathrm{Ca}$, before the formation of regular CAI once the Rayleigh-Taylor fingers have reached the disk.

Supernova injection models do not necessarily suggest that the inner solar system received more ${ }^{26} \mathrm{Al}$ per mass unit than the outer solar system. According to simulations by Vanhala \& Boss (2002), about a dozen Rayleigh-Taylor fingers reach the disk at random locations, separated by $\sim 4 \mathrm{AU}$ on average. Hydrodynamic modeling shows that homogenization of ${ }^{26} \mathrm{Al}$ is a fast process. In a simulation by Boss (2007), material injected at $6 \mathrm{AU}$ spread within the 4-20 AU region in just $10^{3} \mathrm{yr}$. This suggests that ${ }^{26} \mathrm{Al}$ was homogenized rapidly throughout the disk.

Even if ${ }^{26} \mathrm{Al}$ was exclusively formed or injected within the snow line, the Stardust samples from comet 81P/Wild 2 provide undeniable evidence of efficient radial mixing that potentially brought short-lived radionuclides to large heliocentric distances, if they were not there already. About $1-10 \%$ of the $81 \mathrm{P}$ grains (Brownlee et al. 2012) are rich in gehlenitic melilite, anorthite, and fassaitic clinopyroxene - minerals that are typically in CAI. Individual well-studied and nicknamed particles include Inti (Zolensky et al. 2006; Simon et al. 2008), Coki (Matzel et al. 2010), Arthur, and Marvin (Schmitz et al. 2009). Inti is ${ }^{16} \mathrm{O}$ rich, as are most CAI, and its fassaite is rich in trivalent titanium (grossmanite) that only forms in extremely reducing environments $\left(\mathrm{H}_{2}\right.$-dominated, i.e., not in a dust-rich disk midplane), at a level that identifies it as a compact Type A or B CAI. Coki lacks melilite, which is typical of Type C CAI. Condensation temperatures of $>1600 \mathrm{~K}$ for gehlenite and $1416 \mathrm{~K}$ for anorthite (Schmitz et al. 2009) requires formation close to the protosun followed by transport to the primordial disk.

Another group of 81P particles are characterized by lowCa pyroxene (typically $\mathrm{En}_{90 \pm 5} \mathrm{Wo}_{2 \pm 1}$ ) and high-Mg olivine (typically $\mathrm{Fo}_{80-90}$ ) joined in a porphyritic structure, embedded in glassy mesostasis enriched in $\mathrm{SiO}_{2}$ and $\mathrm{Al}_{2} \mathrm{O}_{3}$ (Nakamura et al. 2008; Jacob et al. 2009; Ogliore et al. 2012). This is material typically found in Type I and II olivine-pyroxene chondrules, where the porphyritic structure forms by partial melting of a granular precursor when heated to $1200-1500 \mathrm{~K}$, and the glassy mesostasis is due to rapid cooling. These grains are ${ }^{16} \mathrm{O}$-poor compared to $\mathrm{CAI}$ and have been processed in a more oxidizing and evolved environment (i.e., a dust- and water-rich disk midplane). Bridges et al. (2002) and Joswiak et al. (2014) describe other particles dominated by diogenite $\left(\mathrm{En}_{50} \mathrm{Wo}_{50}\right)$ with properties resembling Al-rich chondrules. These igneous grains must have been transported to the $81 \mathrm{P}$ formation site from the inner solar system and show that an environment fundamentally different from the CAIproducing region provided material to the distant primordial disk as well.

Many $81 \mathrm{P}$ silicate grains are as large as $2-50 \mu \mathrm{m}$ (Brownlee et al. 2012); they display a wide mineralogical range with $\mathrm{Fo}_{4}$ $\mathrm{Fo}_{100}$ olivines that contain up to $6.45 \mathrm{wt} \% \mathrm{MnO}$, high- and low$\mathrm{Ca} \mathrm{En}_{52}-\mathrm{En}_{100}$ pyroxenes (Zolensky et al. 2006), and a crystalline fraction in Fe-bearing silicates of $59_{-10}^{+37} \%$ (Westphal et al. 2009). These crystalline grains are too large to be annealed interstellar grains (these are amorphous $\leq 0.1 \mu \mathrm{m}$ particles, Kemper et al. 2004). They must have formed in a crystalline state by condensation in the solar nebula at $\sim 1100 \mathrm{~K}$ as suggested by their Mn-enrichment (Klöck et al. 1989) that is so variable, compared to the more distinctive $\mathrm{Mn} / \mathrm{Fe}$ ratios of the chondritic meteoritic classes, that the individual grains likely represent a wide range of 
formation distances and times (Brownlee et al. 2012). Similarly, the extreme variability in silicate $\mathrm{Mg} / \mathrm{Fe}$ ratios requires highly diverse temperature and oxygen fugacity conditions (Zolensky et al. 2006), thus formation distances. According to Schmitz \& Brenker (2008) one particular enstatite grain has microstructural characteristics that only form at high temperature $(>1275 \mathrm{~K})$ and fast cooling $\left(>10 \mathrm{~K} \mathrm{~h}^{-1}\right)$. Another grain has so highly reduced forsterite and enstatite that it previously only has been seen in Aubrites, suggesting that E-asteroid material from the inner main belt reached the formation site of 81P (Frank et al. 2013).

The $81 \mathrm{P}$ samples are rich in sulfides such as troilite, pyrrhotite, and pentlandite (Zolensky et al. 2006). Sulfide and/or metallic FeNi grains is the likely host of helium and neon released when $81 \mathrm{P}$ samples are heated above $\sim 1000 \mathrm{~K}$ (Marty et al. 2008). The abundances of the noble gases are so high that implantation by solar wind ion irradiation is the only likely source (Marty et al. 2008), thus the grains were close to the protosun before being transported to the formation site of 81P.

Therefore, consistent lines of evidence show that a wide range of grain types, formed and processed within the snowline, found their way into the primordial disk outside the giant planets in time to be incorporated into comet nuclei. Westphal et al. (2009) estimate that the Stardust sample contains 50-65\% of inner solar system material. This applies for larger particles but is certainly an overestimate for the bulk of solids because sub$\mu \mathrm{m}$ particles largely were destroyed during collection, and those may be dominated by presolar or local mildly processed grains. Observations show that crystalline silicate grains are common in many JFCs (Kelley \& Wooden 2009). Observations of fragments from split comet 73P/Schwassmann-Wachmann 3 (e.g. Harker et al. 2011; Sitko et al. 2011) suggest a crystalline silicate fraction of $25 \%-35 \%$ and there are no evidence that the silicate properties of the deep interior is any different from that of the surface. Combined, this suggests that the mass transport from the inner to the outer solar system was long-lasting and systematic, the source region was extended, and the amount of mass being transported was large. This transport is also expected from numerical modeling (e.g., Shu et al. 1994; Ciesla 2009). It is necessary (e.g., Berger et al. 2011; Ogliore et al. 2012) that this transport took place prior to Jupiter formation at $\sim 3 \mathrm{Myr}$ after CAI because that event terminated radial mixing across the snowline. Thus, transport took place when ${ }^{26} \mathrm{Al}$ had peak energy production. In summary, we consider it difficult to reject the presence of ${ }^{26} \mathrm{Al}$ in the outer solar system and proceed to explain the effect of short-lived radionuclides on icy bodies of various size.

\subsection{Consequences of aluminum-26 in icy bodies}

The effects of radiogenic heating on icy bodies have been studied by many authors (e.g., Prialnik et al. 1987; Haruyama et al. 1993; Yabushita 1993; Prialnik \& Podolak 1995; De Sanctis et al. 2001; Choi et al. 2002; Merk \& Prialnik 2003, 2006; Podolak \& Prialnik 2006; Prialnik et al. 2008). These works illustrate several important general principles. For bodies with identical properties except diameter $D$, small bodies are heated to lower maximum temperatures than large bodies because the surface area is responsible for thermal infrared cooling, while heat production takes place within the volume, the ratio between area and volume being $\propto D^{-1}$. The effect of radiogenic heating is strongly dependent on ice abundance (because radionuclides are only present in refractory dust) and the exact time of formation (because ${ }^{26} \mathrm{Al}$ has a short half-life). A sufficiently high heat conductivity $\kappa$ creates a steep temperature gradient $\frac{\mathrm{d} T}{\mathrm{~d} r} \ll 0$, potentially reaching to the core, leading to an efficient outward heat flux and comparatively low peak temperatures. If steep gradients develop at temperatures sufficiently high to sublimate ice they cause advection, which efficiently transports heat in addition to solid-state conduction. While vapor with sufficiently low condensation temperature may refreeze near the cold surface, other volatiles will be lost to space. However, a sufficiently small $\kappa$ creates a virtually isothermal interior $\left(\frac{\mathrm{d} T}{\mathrm{~d} r} \approx 0\right)$, except for a surface layer that may have a thickness of only meters. This terminates both heat conduction and advection for most of the interior, potentially allowing for conditions where water liquefies (temperature $T \geq 273 \mathrm{~K}$, vapor pressure $p \geq 600 \mathrm{~Pa}$, Prialnik et al. 2008).

Owing to the importance of heat conductivity, it is instructive to investigate the values of $\kappa$ utilized in modeling. The majority of the previously mentioned works applied $\kappa$ and specific heat capacities $c$ from Klinger $(1980,1981)$ and adjusted $\kappa$ for porosity (typically $0.5 \leq \psi \leq 0.8$ ) using the Russell (1935) formula $\Phi_{\mathrm{R}}$. In terms of the thermal inertia $\Gamma=\sqrt{\rho c(1-\psi) \kappa \Phi_{\mathrm{R}}}$, this yields $\Gamma=80-220 \mathrm{MKS}$ and $\Gamma=370-1000 \mathrm{MKS}$ for amorphous $(T=100 \mathrm{~K})$ and crystalline $(T=200 \mathrm{~K})$ ice. These are lower limits since models also include dust. The highest estimate of comet thermal inertia to date is that of Davidsson et al. (2013), who obtained $\Gamma=150-200 \mathrm{MKS}$ in certain regions on 9P/Tempel 1 (but $\Gamma \lesssim 50 \mathrm{MKS}$ elsewhere). These values are consistent with $\Gamma \leq 250 \mathrm{MKS}$ obtained for $103 \mathrm{P} /$ Hartley 2 by Groussin et al. (2013). Compared to $\Gamma=10-50 \mathrm{MKS}$ measured for 67P (Sect. 2.6), and the mean thermal inertia of a sample of Centaurs and TNOs being as low as $\Gamma=2.5 \pm 0.5 \mathrm{MKS}$ (Lellouch et al. 2013), this indicates that most models have applied an effective $\kappa$ that may be 1-3 orders of magnitude too large for comet applications.

We note that Shoshany et al. (2002) formulated an alternative correction formula $\Phi_{\mathrm{S}}$ that yields $\Gamma=30 \mathrm{MKS}$ at $T=100 \mathrm{~K}$ and $\psi=0.72$, if considering composition A with $\{\kappa, \rho, c\}$ as in Sect. 3.3.1. This formula suggests that a thermal inertia of a few tens of MKS may be valid throughout bodies with $\sim 70 \%$ porosity, and considering that gravitational compression is negligible for bodies with $D \lesssim 80 \mathrm{~km}$ (see Sect. 3.3 and, e.g., Prialnik et al. 2008), this might be true for all unprocessed comets and small TNOs. Because heat retainment increases rapidly with decreasing $\kappa$, results from previous modeling may need to be considered as lower limits on the effects of radiogenic heating.

We define "small comets" as having $D \leq 10 \mathrm{~km}$, noting that all but six of the 161 currently known ecliptic comets belong to this category (Lamy et al. 2004; Belton 2014). We define "large comets" as those with $10<D \leq 40 \mathrm{~km}$ plus HaleBopp with $D=74 \pm 6 \mathrm{~km}$ (Szabó et al. 2012). We define "small TNOs" as $40<D \leq 200 \mathrm{~km}$, where the upper limit roughly coincides with the $D=100-190 \mathrm{~km}$ interval where the cumulative size distribution for dynamically hot TNOs changes slope (Fraser et al. 2014). The slope change may reflect a transition between larger primordial bodies and smaller collisionally evolved bodies. With small TNOs we thus focus on thermal evolution in bodies sufficiently small to be potential parents of small and large comets. Our categorization is only made to simplify the description of the size-dependent thermal processing. We define $t_{0}$ as the point in time when aluminum had the canonical ${ }^{26} \mathrm{Al} /{ }^{27} \mathrm{Al}=5 \times 10^{-5}$ composition. 


\subsubsection{Small comets $(D \leq 10 \mathrm{~km})$}

The equilibrium temperature for small dark objects due to solar heating is $\sim 50 \mathrm{~K}$ at $30 \mathrm{AU}$ and $\sim 28 \mathrm{~K}$ at $100 \mathrm{AU}$, suggesting most $\mathrm{CO}, \mathrm{CH}_{4}$, and $\mathrm{N}_{2}$ not trapped within amorphous water ice may have been lost in $\mathrm{EKB} / \mathrm{SD}$ comets even in the absence of radionuclides.

Small comets with $D \gtrsim 1 \mathrm{~km}$ formed at $t_{0}$ lose $\mathrm{CO}, \mathrm{CO}_{2}$, and amorphous water ice, and $D \gtrsim 8 \mathrm{~km}$ nuclei have $\gtrsim 10 \%$ liquid water in the core (Prialnik et al. 1987; Merk \& Prialnik 2003, 2006; Podolak \& Prialnik 2006). If the ice abundance is $\sim 25 \%$ nuclei with $D \gtrsim 8 \mathrm{~km}$ formed at $t_{0}$ reach $360 \mathrm{~K}$ and may disrupt entirely by gas pressure (Merk \& Prialnik 2006). Because $D \gtrsim$ $4 \mathrm{~km}$ nuclei also reach a high core temperature $(\sim 320 \mathrm{~K})$ they may disrupt as well.

If formation of $D \approx 4 \mathrm{~km}$ nuclei is delayed by $\sim 1.5 \mathrm{Myr}$ $(\Gamma \approx 500 \mathrm{MKS}$, Prialnik et al. 1987$)$ to $\sim 4.7 \mathrm{Myr}(\Gamma \approx 3 \mathrm{MKS}$, Haruyama et al. 1993), crystallization will be avoided. The necessary delay is $\sim 1.8 \mathrm{Myr}$ at $D \approx 1 \mathrm{~km}$ and $\sim 6.7 \mathrm{Myr}$ at $D \approx 10 \mathrm{~km}$, assuming a very low conductivity (Haruyama et al. 1993). However, for the largest nuclei in this category crystallization is likely even in the absence of ${ }^{26} \mathrm{Al}$, if the dust abundance is sufficiently large (because of ${ }^{40} \mathrm{~K}$ decay).

\subsubsection{Large comets $(10<D \leq 40 \mathrm{~km})$}

While $D=20 \mathrm{~km}$ objects are heated globally by sunlight to $50 \mathrm{~K}$ at $30 \mathrm{AU}$ in just $\sim 1 \mathrm{Myr}$, they are small enough to dissipate heat sufficiently well to not experience additional heating if only long-lived radionuclides are present (Choi et al. 2002). Thus, $\mathrm{N}_{2}, \mathrm{CO}$, and $\mathrm{CH}_{4}$ may be lost, unless being trapped in amorphous water ice, but $\mathrm{CO}_{2}$ and $\mathrm{NH}_{3}$ could still survive as pure ice (De Sanctis et al. 2001).

If formed at $t_{0}$ and relatively dust-rich, these bodies may develop sufficient vapor pressure compared to self gravity to disrupt (Merk \& Prialnik 2006). A higher ice abundance ( 50\%) produces a completely liquefied body that may remain intact, containing liquid water for up to $\sim 4.5 \mathrm{Myr}$ (Merk \& Prialnik 2003, 2006).

If formed with a $\sim 2.5 \mathrm{Myr}$ delay these bodies crystallize (if containing amorphous ice), thus lose most their $\mathrm{CO}$ and $\mathrm{CO}_{2}$ except for a $\sim 1 \mathrm{~km}$ surface layer (Prialnik \& Podolak 1995; Choi et al. 2002; Merk \& Prialnik 2003, 2006; Prialnik et al. 2008). To avoid crystallization in $D \approx 40 \mathrm{~km}$ comets a formation delay of $\sim 4.5 \mathrm{Myr}$ ( $\Gamma \approx 500 \mathrm{MKS}$, Prialnik et al. 1987 ) to $\sim 9.7 \mathrm{Myr}$ $(\Gamma \approx 3$ MKS, Haruyama et al. 1993$)$ is necessary.

\subsubsection{Small TNOs $(40<D \leq 200 \mathrm{~km})$}

These bodies are sufficiently large that long-lived radionuclides are capable of causing crystallization and loss of most $\mathrm{N}_{2}, \mathrm{CO}$, and $\mathrm{CO}_{2}$, except for a thin surface region if the conductivity is low (Yabushita 1993). If the conductivity is higher, and especially if advection takes place, they may remain amorphous in the unlikely scenario that ${ }^{26} \mathrm{Al}$ was absent (Prialnik et al. 1987; Choi et al. 2002).

If formed at $t_{0}$ the interiors will be liquid and self-gravity is sufficient to avoid steam pressure disruption. An outer $\sim 1 \mathrm{~km}$ layer of amorphous ice with supervolatiles is likely (Podolak \& Prialnik 2006; Merk \& Prialnik 2003; Prialnik et al. 2008). A delayed formation (by 10-20 Myr) will allow for a thicker shell (comprising 20\%-50\% of the radius) of amorphous water ice (Merk \& Prialnik 2003, 2006). However, much of this may be lost over time due to a combination of long-lived radioactivity and solar heating (Yabushita 1993; De Sanctis et al. 2001).

\subsubsection{Consequences of thermal processing}

The presence of ${ }^{26} \mathrm{Al}$ may therefore cause considerable thermal processing. Large comets and small TNOs formed sufficiently early (within 1-20 Myr after $t_{0}$, depending on $D$ ) will contain significant amounts of liquid water. This leads to aqueous alteration (formation of phyllosilicates) and metasomatism (formation of secondary minerals other than phyllosilicates). Furthermore, there is a substantial loss of porosity, thus higher bulk densities.

Body cores that are heated above $\sim 80 \mathrm{~K}$ will sublimate $\mathrm{CO}_{2}$ and $\mathrm{NH}_{3}$ (heating to $\sim 140 \mathrm{~K}$ may be necessary to lose these species if they primarily are trapped in amorphous water ice). To avoid $T \gtrsim 80 \mathrm{~K}$ in $D=10-20 \mathrm{~km}$ objects, they can form no earlier than $\sim 4.5 \mathrm{Myr}$ after $t_{0}$ (Prialnik et al. 1987). A comet like Hale-Bopp requires a substantially later formation, perhaps 10-20 Myr after $t_{0}$. We note that there have been claims of amorphous water ice in Hale-Bopp (Davies et al. 1997) and in C/2002 T7 LINEAR (Kawakita et al. 2004) based on the spectra of icy coma grains. If this is correct, and if ${ }^{26} \mathrm{Al}$ indeed was common in the outer solar system, this implies a rather late formation of Hale-Bopp. Astrophysical observations show that about half of the solar-mass protostars have lost their gas disks after just 3 Myr (Zuckerman et al. 1995; Haisch et al. 2001; SiciliaAguilar et al. 2006). A substantial part of the growth of HaleBopp to its current size may have taken place after the solar nebula dispersed.

For comets experiencing $\mathrm{CO}_{2}$ and $\mathrm{NH}_{3}$ mobilization, some may be lost to space, while most may recondense near the cold surface. Doing so in equilibrium conditions would likely produce ice in which the ortho-to-para ratio (OPR) of $\mathrm{NH}_{3}$ corresponds to a spin temperature $T_{\text {spin }}$ near the condensation temperature, thus $T_{\text {spin }} \approx 80 \mathrm{~K}$. If $\mathrm{N}_{2}, \mathrm{CO}$, and $\mathrm{CH}_{4}$ are liberated through crystallization of water ice in the core of a small body (e.g., a $D=4 \mathrm{~km}$ nucleus formed prior to $1.5-4.7 \mathrm{Myr}$ after $t_{0}$ ) the vapor would only refreeze as pure ice near the surface, if the object is $\gtrsim 200 \mathrm{AU}$ from the Sun. If so, the spin temperatures would probably be $T_{\text {spin }} \approx 20-30 \mathrm{~K}$.

\subsection{Evidence of thermal processing from density and shape}

The effect of short-lived radionuclides on the interior structure of regular Saturnian satellites have been explored, e.g., by CastilloRogez et al. (2007, 2009) for Iapetus, by Czechowski (2012) for Rhea, and by Schubert et al. (2007) for Enceladus. Among these, Iapetus appears to provide the strongest support for the presence of ${ }^{26} \mathrm{Al}$ beyond the snowline and Castillo-Rogez et al. (2007, 2009) constrain the formation time of Iapetus to 3.4-5.4 Myr after CAI.

The irregular Saturnian satellite Phoebe was most likely captured into its current retrograde orbit from the primordial disk. It did not necessarily form in the vicinity of Saturn, but may have been scattered inwards from larger distances by the ice giants. Because $\rho=1634 \pm 46 \mathrm{~kg} \mathrm{~m}^{-3}$ (Matson et al. 2009), composition A suggests an average porosity of just $10 \%$. However, for the weak self gravity of a $D=217.7 \pm 1.5 \mathrm{~km}$ body, pressureporosity relations for granular material predict a porosity of $\sim 35 \%$ at the center and $\sim 50 \%$ near the surface (Castillo-Rogez et al. 2012). Despite the small size the shape is close to an oblate ellipsoid with axis ratio 0.93 . This suggests that an initially very 
porous Phoebe was heated radiogenically to the point of compaction, and the surface assumed its current shape. Modeling by Castillo-Rogez et al. (2012) shows that long-lived radioactivity is not sufficient to crystallize amorphous ice, and such a body will remain highly porous although it relaxes to an oblate shape due to the low viscosity of amorphous ice. If the ice is crystalline to begin with, long-lived radioactivity may compact the central region. However, the outer region remains porous and the body does not relax to an oblate shape. None of these cases are satisfactory representations of Phoebe. If ${ }^{26} \mathrm{Al}$ is added, the necessary compaction and reshaping of the body is readily achieved and it is likely that differentiation takes place. The observed properties of Phoebe may therefore require ${ }^{26} \mathrm{Al}$ heating.

Another object that shares some of Phoebe's properties is the jovian irregular satellite Himalia. With dimensions $120 \pm 20 \mathrm{~km}$ by $150 \pm 20 \mathrm{~km}$ (Porco et al. 2003) from Cassini imaging, and a mass of $4.2( \pm 0.6) \times 10^{18} \mathrm{~kg}$ (Emelyanov 2005) from its perturbation on the satellite Elara, the nominal density estimate is $\rho=2400 \mathrm{~kg} \mathrm{~m}^{-3}$ with a lower limit $\rho \geq 1400 \mathrm{~kg} \mathrm{~m}^{-3}$. The high density for such a small icy object suggests significant compaction by radiogenic heating, that may require ${ }^{26} \mathrm{Al}$.

\subsubsection{The cold classical EKB}

We here propose an observational test that may reveal whether dynamically cold classical EKB objects (inclination $i \lesssim 5^{\circ}$, eccentricity $e \lesssim 0.2$ ) contained ${ }^{26} \mathrm{Al}$ at birth. These objects may have formed at $r_{\mathrm{h}} \approx 25-30 \mathrm{AU}$ and then been scattered outward by Neptune because only distant bodies maintain dynamically cold orbits during the transfer (Gomes 2003). However, $22_{-5}^{+10} \%$ of these objects are (ultra)wide binaries (Stephens \& Noll 2006) and their low probability of survival during scattering suggests in situ formation (Parker \& Kavelaars 2010; Parker et al. 2011). Gravitational collapse of pebble swarms (Nesvorný et al. 2010) created by streaming instabilities (Youdin \& Goodman 2005; Johansen et al. 2012) is the only known mechanism that readily creates large numbers of (ultra)wide binaries.

Pebble swarms form during $\sim 10$ orbits (Johansen et al. 2007) and the cold classical binaries formed in a relatively narrow $r_{\mathrm{h}^{-}}$ range. The fast formation in a small region of space suggests that all bodies obtain similar ice and rock fractions. Collision velocities during assembly are $v_{\text {coll }} \approx 10 \mathrm{~m} \mathrm{~s}^{-1}$ (Nesvorný et al. 2010; Wahlberg Jansson \& Johansen 2014). That leads to formation of very porous bodies. Subsequent accretion of viscously stirred planetesimals (impacting at $\geq 100 \mathrm{~m} \mathrm{~s}^{-1}$ ) causes substantial collisional compaction (Sect. 3.5). However, projectiles as small as $1-5 \mathrm{~km}$ are capable of unbinding wide binaries (Parker \& Kavelaars 2012), suggesting that accretion and collisional compaction did not take place for this particular population. Therefore, newly born wide binaries should be characterized by very low bulk densities.

We calculate initial bulk densities as follows. A pressureporosity relation $p=p(\psi)$ at $p \leq 0.23 \mathrm{MPa}$ is defined as an average between that of pure $\mu \mathrm{m}$-sized silica dust (Güttler et al. 2009) and that of pure $\mu \mathrm{m}$-sized water ice grains (Lorek et al. 2016), weighted by volume. At $p>0.23 \mathrm{MPa}$ we apply the $p=p(\psi)$ relation measured by Yasui \& Arakawa (2009) for ice with 29\% dust. A computer code calculates the gravitational pressure at each point within a spherical body based on its internal mass distribution, which is updated iteratively as an initially very porous body is being compressed under self-gravity. Code accuracy was verified by reproducing the hydrostatic equilibrium porosity profiles of Henke et al. (2012). To account for non-zero accretion velocities, a dynamic impact pressure $p_{\text {imp }}=\rho v_{\text {coll }}^{2} / 2$ (e.g.,

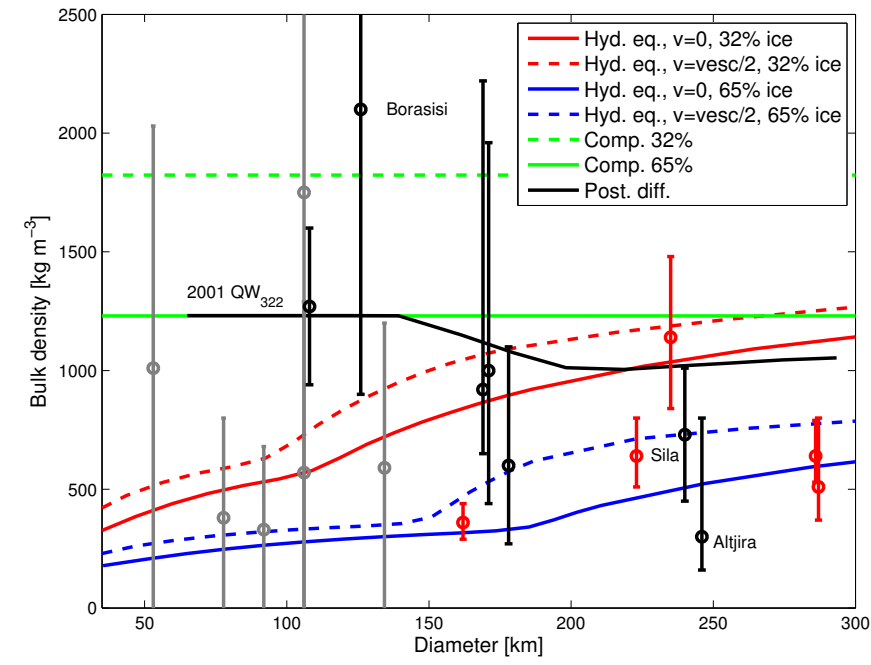

Fig. 10. Red and blue curves: hydrostatic equilibrium bulk porosities for composition A ( $32 \%$ ice) and B (65\% ice). The solid curves assume $v_{\text {coll }}=0$ and the dashed assume $v_{\text {coll }}=v_{\text {esc }} / 2$. The green lines are the fully compressed densities. The black curve is obtained after thermal processing of bodies with $65 \%$ ice formed with $v_{\text {coll }}=v_{\text {esc }} / 2$. Measured densities for dynamically cold classical disk objects (black, gray) are taken from Petit et al. (2008), Vilenius et al. (2012, 2014), and Carry (2012) based on Grundy et al. (2009, 2013). Densities for dynamically hot classical disk objects, SD objects, and Centaurs (red) are taken from Spencer et al. (2006), Benecchi et al. (2010), Mommert et al. (2012), Santos-Sanz et al. (2012), and Vilenius et al. (2014).

Blum \& Wurm 2008) can be added to the gravitational pressure. We here assume $\rho=300 \mathrm{~kg} \mathrm{~m}^{-3}$ for accreting pebbles and that $v_{\text {coll }}$ equals half the escape velocity $v_{\text {esc }}$ of the body being formed. On the $65 \leq D \leq 250 \mathrm{~km}$ interval $v_{\text {coll }}$ grows from $\sim 7 \mathrm{~m} \mathrm{~s}^{-1}$ to $\sim 25 \mathrm{~m} \mathrm{~s}^{-1}$, which is in line with simulations of pebble-swarm collapse.

Figure 10 shows $\rho_{\text {bulk }}$ versus $D\left(v_{\text {coll }}=0\right)$ for $f_{\text {ice }}=32 \%$ and $f_{\text {ice }}=65 \%$ as solid red and blue curves (i.e., composition A and B). The corresponding curves for $v_{\text {coll }}=v_{\text {esc }} / 2$ are dashed. As expected, $\rho_{\text {bulk }}$ gradually increases with $D$. The internal porosity distributions change dramatically on the $65 \leq D \leq 250 \mathrm{~km}$ interval due to the increasing importance of self-gravity. If $f_{\text {ice }}=65 \%$ and $v_{\text {coll }}=v_{\text {esc }} / 2$, a $D=65 \mathrm{~km}$ body has $\psi=72 \%$ at the center and $\psi=75 \%$ at the surface. However, a $D=250 \mathrm{~km}$ body has $\psi=31 \%$ at the center and $\psi=45 \%$ at the surface. Because $\kappa$ is a non-linear function of $\psi$ (Sect. 3.2) the two bodies will have significantly different thermal evolution, if heated by ${ }^{26} \mathrm{Al}$. Using the "generation 1" version of the Shoshany et al. (2002) function $\Phi_{\mathrm{S}}$, it predicts $\Phi_{\mathrm{S}}=3.7 \times 10^{-5}$ for $\psi=72 \%$ and $\Phi_{\mathrm{S}}=0.45$ for $\psi=31 \%$, that is, the heat conductivities differ a factor of $1.2 \times 10^{4}$ between $D=65 \mathrm{~km}$ and $D=250 \mathrm{~km}$ bodies.

We calculate thermal evolution as follows. We assume heat conductivities and heat capacities ${ }^{3}$ such that $\Gamma=30 \mathrm{MKS}$ at $\psi=72 \%$. We here consider composition B with $v_{\text {coll }}=v_{\text {esc }} / 2$. A computer code calculates the temperature as function of radial distance $r$ from the center and time, accounting for ${ }^{26} \mathrm{Al}$ heating (starting at $20 \%$ of the canonical value), heat conduction,

3 Compact rock/metal grains have $\kappa_{\mathrm{d}}=31 \mathrm{~W} \mathrm{~m}^{-1} \mathrm{~K}^{-1}, \rho_{\mathrm{d}}=$ $3840 \mathrm{~kg} \mathrm{~m}^{-3}$, and $c_{\mathrm{d}}=690 \mathrm{~J} \mathrm{~kg}^{-1} \mathrm{~K}^{-1}$ when applying volume-weighted averages for enstatite and iron. Compact icy grains have $\kappa_{\mathrm{i}}=$ $3.5 \mathrm{~W} \mathrm{~m}^{-1} \mathrm{~K}^{-1}, \rho_{\mathrm{i}}=917 \mathrm{~kg} \mathrm{~m}^{-3}$, and $c_{\mathrm{i}}=2100 \mathrm{~J} \mathrm{~kg}^{-1} \mathrm{~K}^{-1}$. For the mixtures composition-weighted averages $\left\{\kappa_{\text {mix }}, \rho_{\text {mix }}, c_{\text {mix }}\right\}$ are used. Heat capacity and heat conductivity are corrected for porosity as $c_{\text {mix }} \rho_{\text {mix }}(1-$ $\psi)$ and $\kappa_{\operatorname{mix}} \Phi_{\mathrm{S}}$. 
and radiative cooling from the surface (initial temperature $T_{0}=$ $30 \mathrm{~K}$ ). Modeling proceeds for $10 \mathrm{Myr}$. We identify regions that have reached water melting at some point and collapse those to zero porosity, while exterior regions have porosity in accordance with the gravity field. The performance of the thermal code was validated in the case of constant $\psi$ by reproducing temperature profiles from Miyamoto et al. (1981), while profiles from Hevey $\&$ Sanders (2006) were used for validation when $\psi=\psi(r)$.

The result is plotted as a black curve in Fig. 10. Surprisingly, small bodies become more compact than large ones. A $D=$ $60 \mathrm{~km}$ body has such a small $\kappa$ that heat efficiently is trapped and ice melting is reached everywhere except for the top $16 \mathrm{~m}$. It becomes fully compacted and differentiated, reaching $\rho_{\text {bulk }}=$ $1230 \mathrm{~kg} \mathrm{~m}^{-3}$. During shrinkage, the outer crust may break and mix with mantle water. A $D=250 \mathrm{~km}$ body cools less efficiently than a $D=60 \mathrm{~km}$ body. However, this is overcompensated for by the higher heat conductivity of its comparatively compact interior. In this case, the outer $17 \mathrm{~km}$ remains unmolten and partial compaction yields $\rho_{\text {bulk }}=1030 \mathrm{~kg} \mathrm{~m}^{-3}$. During shrinkage the thick crust may prevent liquid water from reaching the surface. The slope of the black curve in Fig. 10 will steepen if the smaller objects experience a significant loss of volatiles.

Figure 10 also shows density versus primary diameters for wide binaries in the cold classical EKB. The targets in black were observed with Herschel by Vilenius et al. (2012, 2014) and have radiometrically determined sizes and geometric albedos (except $2001 \mathrm{QW}_{322}$ for which Petit et al. 2008 assumed $\left.p_{\mathrm{V}}=0.16\right)$. For the targets in gray Grundy et al. $(2009,2013)$ assumed ranges for $\rho_{\text {bulk }}$ and $p_{\mathrm{V}}$ to constrain diameters. Carry (2012) then used these diameters and measured masses to estimate $\rho_{\text {bulk }}$. We consider the black targets more reliable than the gray ones. Because all objects of a given ice abundance must be at or above the corresponding equilibrium curve, (79360) Sila and (148780) Altjira suggest that $f_{\text {ice }} \approx 60 \%$ is more likely than $f_{\text {ice }} \approx 30 \%$. For the black points there is a tendency for $\rho_{\text {bulk }}$ to decrease with increasing $D$. Thus, they do not follow the hydrostatic equilibrium curves for a granular ice-dust mixture. The decrease takes place in the size range where strong initial differences in porosity are expected. A possible explanation for the slope is therefore thermal evolution by ${ }^{26} \mathrm{Al}$ decay (long-lived radionuclides do not suffice), that affects small high-porosity bodies more strongly than large low-porosity bodies.

Interestingly, the geometric albedos are decreasing with increasing $D$ for these objects. (66652) Borasisi has $p_{\mathrm{V}}=0.236$, the three objects near $D=170 \mathrm{~km}$ ((88611) Teharonhiawako, $2001 \mathrm{XR}_{254}$, (275809) $2001 \mathrm{QY}_{297}$ ) all have $0.136 \leq p_{\mathrm{V}} \leq$ 0.152 , while (79360) Sila have $p_{\mathrm{V}}=0.09$ and (148780) Altjira $p_{\mathrm{V}}=0.043$ (Vilenius et al. 2012, 2014). We propose that this albedo slope is a consequence of differentiation that has brought water to the surface of the smaller targets, while the large ones may have more pristine crusts.

\subsection{Aqueous alteration in the outer solar system}

If primordial disk planetesimals contained liquid water, aqueous alteration has taken place. The irregular giant planet satellites, Centaurs, EKB, and SD objects should therefore contain hydrated materials that, if present on their surfaces, give rise to characteristic absorption bands. The $0.7 \mu \mathrm{m}$ phyllosilicate absorption band has been seen in 11 out of 16 studied jovian irregular satellites, including Himalia (Jarvis et al. 2000; Vilas et al. 2006). Some of these (Callirrhoe, Megaclite, and Themisto) are comet-sized bodies with $D=6-8 \mathrm{~km}$ (Sheppard \& Jewitt 2003). Out of eight observed Saturnian irregular satellites,
Vilas et al. (2006) detected $0.7 \mu \mathrm{m}$ absorption only in Phoebe. Cassini observations of Phoebe by Clark et al. (2005) confirm this absorption and also revealed phyllosilicate metal-OH group absorption at $2.16 \mu \mathrm{m}$ and $2.3 \mu \mathrm{m}$, as well as a $2.72 \mu \mathrm{m} \mathrm{OH}$ stretch fundamental common to phyllosilicates and a $2.95 \mu \mathrm{m}$ feature from trapped $\mathrm{H}_{2} \mathrm{O}$ or water ice. Furthermore, absorption at $1.5 \mu \mathrm{m}$ and $1.95 \mu \mathrm{m}$ due to bound water may indicate hydrated minerals.

Among more distant bodies, the Uranian irregular satellite Caliban has $0.7 \mu \mathrm{m}$ absorption (Vilas et al. 2006), and weak features were also seen in the Centaur (10199) Chariklo (Lederer $\&$ Vilas 2004) and in the plutinos 2003 AZ $_{84}$ (Fornasier et al. 2004), $2000 \mathrm{GN}_{171}$, and $2000 \mathrm{~EB}_{173}$ (Lazzarin et al. 2003). Observations of $2000 \mathrm{~EB}_{173}$ (de Bergh et al. 2004) show a possible band at $2.28 \mu \mathrm{m}$, coinciding with absorption of $\mathrm{OH}$ bearing Al, Mg-rich minerals. That band, and an associated one at $1.4 \mu \mathrm{m}$, were seen by Jewitt \& Luu (2001) in the Centaur $1999 \mathrm{DE}_{9}$.

Takir \& Emery (2012) measured the 0.8-2.5 $\mu \mathrm{m}$ and 1.9$4.1 \mu \mathrm{m}$ spectra of 28 outer main belt asteroids. They distinguished between four different types of $3 \mu \mathrm{m}$ features, of which the "sharp" feature is due to phyllosilicates and the "rounded" feature is due to water ice. The fraction of asteroids displaying a "sharp" $3 \mu \mathrm{m}$ feature decreases with increasing $r_{\mathrm{h}}$. In their view, distant asteroids formed late when most ${ }^{26} \mathrm{Al}$ had decayed, therefore these bodies lack the "sharp" $3 \mu \mathrm{m}$ feature. If so, one might expect little to no thermal processing in the primordial disk, where the bodies may have formed even later. However, we propose an alternative interpretation. Thermophysical models (e.g., Hevey \& Sanders 2006; Henke et al. 2012) show that the crust of radiogenically heated bodies remains cold and unprocessed even when the interior is molten. To bring phyllosilicates to the surface and give the body a "sharp" $3 \mu \mathrm{m}$ feature, parts of the crust must be removed. This requires efficient impact gardening. Because the collision frequency decreases with increasing $r_{\mathrm{h}}$, this might explain why the "sharp" $3 \mu \mathrm{m}$ feature becomes less common at large distances. The key fact is that the phyllosilicate absorption feature does not disappear entirely but is present throughout the solar system. This means that all of these bodies may have been thermally processed, though only few have enough phyllosilicates on their surfaces to give rise to observable absorption.

Therefore, we do not doubt that aqueous alteration took place in the large bodies of the outer solar system. Comets, however, appear to be different. The Stardust samples have been searched for phyllosilicates, yet none has been found (Stodolna et al. 2010, 2012; Berger et al. 2011; Brownlee et al. 2012; Joswiak et al. 2012). Laboratory experiments of the capture process indicate that phyllosilicates have a substantial chance of survival (Noguchi et al. 2007; Foster et al. 2008; Wozniakiewicz et al. 2010), suggesting these minerals should have been found if they are common (Joswiak et al. 2012). In addition, 81P contains silica grains (crystalline cristobalite) on a $\sim 2 \%$ level (Roskosz \& Leroux 2015). They probably form during low-temperature annealing of amorphous silicates (Roskosz \& Leroux 2015). The observationally established presence of silica at a $2-10 \%$ level in protostellar disks combined with their presence in $81 \mathrm{P}$ but absence in ordinary and carbonaceous chondrites (where liquid water quickly destroys silica) suggest that $81 \mathrm{P}$ was not a host of in situ aqueous alteration. Yet, the Stardust samples contain examples of grains made of magnetite (Stodolna et al. 2010, 2012), carbonate (Flynn et al. 2009), cubanite $\left(\mathrm{CuFe}_{2} \mathrm{~S}_{3}\right)$, sphalerite $((\mathrm{Fe}, \mathrm{Zn}) \mathrm{S})$, and Ni-bearing pyrrhotite (Berger et al. 2011). These minerals are known to form during aqueous alteration, although 
magnetite, $\mathrm{Zn}$, Ni-bearing sulfides, and carbonates may form in other ways (Flynn et al. 2009; Joswiak et al. 2012). However, this aqueous alteration is believed to have taken place prior to inclusion into 81P (Stodolna et al. 2010).

The Stardust results differ from those inferred from Spitzer observations of comet 9P/Tempel 1 ejecta during the Deep Impact experiment. Lisse et al. (2006) find that $\sim 8 \%$ of the phyllosilicate nontronite (a smectite) is necessary to fully explain the spectral shape in the $20-25 \mu \mathrm{m}$ region, that is dominated by olivine and pyroxene emission features. The phyllosilicates saponite and serpentine do not match. Nontronite is the only important host of sodium in the spectral fitting. Nontronite is also necessary to fit the spectrum of Hale-Bopp (Lisse et al. 2007). Compared to the mineralogies of $81 \mathrm{P}$ and meteorites these results are very surprising. In Stardust samples sodium is partitioned between Na-rich clinopyroxene and the feldspar albite, in proportions that suggest cooling and crystallization of a CMAS melt (Ca, Mg, Al, Si oxides), and albite would likely have dominated had it not been for the presence of $\mathrm{Cr}$ in the melt (Joswiak et al. 2009). This is similar to matrix in the most unequilibrated (petrographic grade 3.0) water-poor enstatite chondrites (e.g., Rubin et al. 2009) and ordinary chondrites (Scott et al. 1984; Brearley 1989; Brearley et al. 1989) where albite (or albitic glass) is the primary Na-carrier. In the more waterrich carbonaceous chondrites even grade 3.0 meteorites have not managed to avoid metasomatism, leading to replacement of albite by secondary minerals. This process systematically forms the feldspathoids nepheline and (less) sodalite that are the major Na-carrier in CO3 (Tomeoka \& Itoh 2004) and CV3 (Krot et al. 1995) carbonaceous chondrites. Aqueous alteration (grade 1-2 CI, CM, CR carbonaceous chondrites) of albite, nepheline and sodalite produces Na-rich phyllosilicates, where saponite dominates completely and serpentines are common (Rubin 1997). The only smectite regularly encountered is montmorillonite (Rubin 1997). Nontronite is common on Mars where it forms when water interacts with basalt and gabbro (Poulet et al. 2005). It is unclear why nontronite should be a major mineral phase on some comet nuclei when it is extremely rare or absent in aqueously altered carbonaceous chondrites, as well as in 81P and (most likely) 67P. It is likely that differences between the comet and the laboratory samples used by Lisse et al. (2006) in terms of particle size distribution, grain shapes, and grain cluster porosities for pyroxene and olivine, are responsible for the spectral differences. If so, nontronite may not be necessary to explain the observed spectra and the fit is not unique.

The OPRs and spin temperatures further support the notion that comet nuclei did not experience strong heating by radionuclides. If sublimation and recondensation of ammonia took place $T_{\text {spin }} \approx 80 \mathrm{~K}$ may be expected (Sect. 3.2.4). However, Kawakita et al. (2006) list six comets for which the $\mathrm{NH}_{3}$ spin temperature has been measured and the nominal values are in the range $25 \leq T_{\text {spin }} \leq 32 \mathrm{~K}$. Specifically, Hale-Bopp has $T_{\text {spin }}=26_{-4}^{+10} \mathrm{~K}$. This may suggest that an object even as large as Hale-Bopp did not reach the sublimation temperature of $\mathrm{NH}_{3}$, alternatively, it did not reach the crystallization temperature of amorphous water ice. Furthermore, the spin temperature of water is in the range $23 \leq T_{\text {spin }} \leq 36 \mathrm{~K}$ for six comets, and comet $\mathrm{C} / 2001 \mathrm{Q} 4$ NEAT has a methane spin temperature of $T_{\text {spin }}=33_{-1}^{+2} \mathrm{~K}$. Kawakita et al. (2006) suggest that the spin temperature of the three species (consistently indicating $T_{\text {spin }} \approx 30 \mathrm{~K}$ ) may reflect the grain temperature in the presolar molecular cloud (onto which $\mathrm{H}_{2} \mathrm{O}, \mathrm{NH}_{3}$, and $\mathrm{CH}_{4}$ condensed). If so, significant sublimation and refreezing may not have been taking place. There may be some exceptions, for example C/1986 P1 Wilson that has a
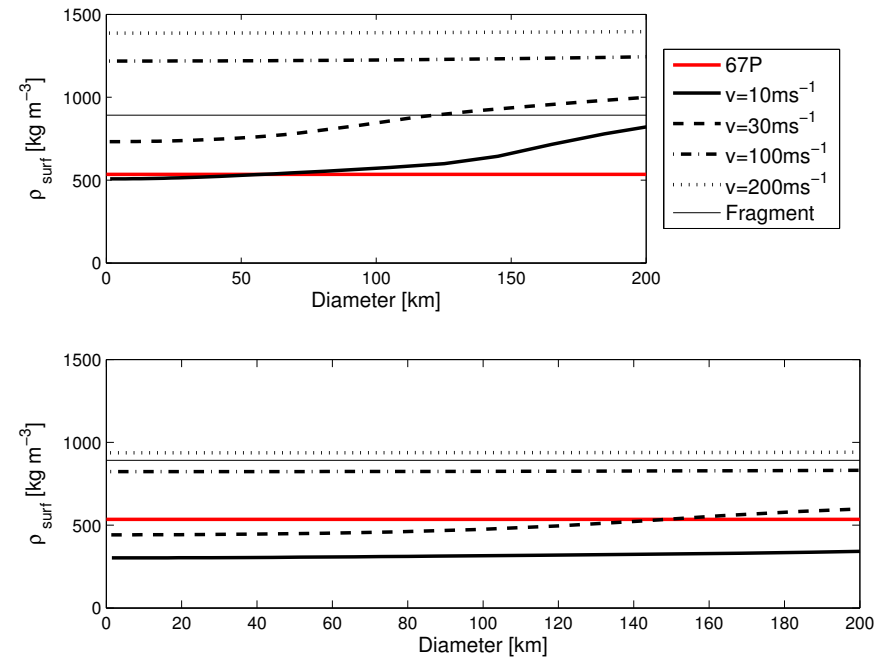

Fig. 11. Average density $\rho_{\text {surf }}$ in the outer $20 \%$ by radius (50\% by mass) of bodies with diameter $D$ grown at various accretion velocities, for composition A (upper panel) and composition B (lower panel), using the method described in Sect. 3.3.1. The curves can be compared to the bulk density $\rho_{\text {bulk }}$ of 67P (red) and fragment density $\rho_{\text {frag }}$ (thin black solid line) needed in combination with a $40 \%$ macroporosity to give $\rho_{\text {bulk. }}$.

lower limit of $T_{\text {spin }} \geq 50 \mathrm{~K}$ for water (Kawakita et al. 2006) and 73P/Schwassmann-Wachmann 3 that has a lower limit of $T_{\text {spin }} \geq 39 \mathrm{~K}$ for $\mathrm{NH}_{3}$ (Shinnaka et al. 2011).

\subsection{Collisional processing}

During the $\sim 400$ Myr lifetime of the primordial disk the collision velocities slowly increased with time due to viscous stirring, reaching $\sim 280-740 \mathrm{~m} \mathrm{~s}^{-1}$ at $t=400 \mathrm{Myr}$ according to Sect. 4.4 . These are non-destructive collisions for TNO-sized bodies but lead to compaction. We here apply the same compositions and $p=p(\psi)$ relation as in Sect. 3.3.1 but focus on the effect of elevated accretion velocities for the average density $\rho_{\text {surf }}$ in the outer $20 \%$ by radius (outer $50 \%$ by mass).

Figure 11 shows $\rho_{\text {surf }}$ versus $D$ for different accretion velocities. We first note that accretion velocities in the range 10 $30 \mathrm{~m} \mathrm{~s}^{-1}$ appear optimal for obtaining the 67P density. That corresponds to $p_{\text {imp }} \approx 15-135 \mathrm{kPa}$, suggesting that the compressive strength $S_{\mathrm{c}}$ takes similar values. This is intermediate between the small/large-scale values for 67P (Sect. 2.2) and is overlapping the $S_{\mathrm{t}} \approx 0.4-34 \mathrm{kPa}$ measured for debris in cometary meteor showers (Trigo-Rodríguez \& Llorca 2006).

Figure 11 also shows that $>30 \mathrm{~m} \mathrm{~s}^{-1}$ accretion causes significant compaction. A small TNO that accretes $\sim 100 \mathrm{~m} \mathrm{~s}^{-1}$ planetesimals here obtains $\rho_{\text {surf }}=800-1200 \mathrm{~kg} \mathrm{~m}^{-3}$ (depending on composition). At $\sim 200 \mathrm{~m} \mathrm{~s}^{-1}$ the range is $\rho_{\text {surf }}=950$ $1400 \mathrm{~kg} \mathrm{~m}^{-3}$ and at $\sim 300 \mathrm{~m} \mathrm{~s}^{-1}$ it is $\rho_{\text {surf }}=1000-1500 \mathrm{~kg} \mathrm{~m}^{-3}$. Asteroid (253) Mathilde (Housen et al. 1999) and Saturnian satellite Hyperion (Housen \& Holsapple 2012) may serve as examples of porous objects in the process of being collisionally compacted. Statistically, a large number of small compressive impacts takes place before collision with a projectile sufficiently large to disrupt the body. The asteroid (25143) Itokawa shows that the macroporosity in small $(0.54 \times 0.30 \times 0.24 \mathrm{~km})$ collisional rubble-piles is only $40 \%$ (Abe et al. 2006). Thus, if $67 \mathrm{P}$ is a collisional rubble-pile its fragments need a density $\rho_{\text {frag }} \approx 860 \mathrm{~kg} \mathrm{~m}^{-3}$ (thin black line in Fig. 11) to explain its measured $\rho_{\text {bulk }}$. Comparing $\rho_{\text {frag }}$ to $\rho_{\text {surf }} \gtrsim 1000-1500 \mathrm{~kg} \mathrm{~m}^{-3}$ 
(or to composition A with $40 \%$ porosity, $\rho \approx 1100 \mathrm{~kg} \mathrm{~m}^{-3}$ ) suggests that it is not trivial to disrupt a thermally and collisionally compacted small TNO and form a low-density comet from the rubble.

The dynamically hot EKB population presently has average collision velocities of $\sim 1.65 \mathrm{~km} \mathrm{~s}^{-1}$, peaking at $\sim 2.69 \mathrm{~km} \mathrm{~s}^{-1}$ in the inner parts of the belt (Dell'Oro et al. 2013). This is much higher than in the primordial disk and may lead to significant collisional damage. Therefore, collisions among differentiated TNOs (Sect. 3.2) may have created fragments of almost pure ice or rock. The Themis family in the outer asteroid main belt may exemplify such collisions. Based on density differences within this family Castillo-Rogez \& Schmidt (2010) propose breakup of a differentiated parent. This is exemplified by the members (24) Themis $\left(D \approx 198 \mathrm{~km}, \rho_{\text {bulk }}=2780 \pm 1060 \mathrm{~kg} \mathrm{~m}^{-3}\right.$, Baer $\&$ Chesley 2008) and (90) Antiope (a binary with $D_{1}=88 \mathrm{~km}$, $D_{2}=84 \mathrm{~km}$ and $\rho_{\text {bulk }}=1250 \pm 50 \mathrm{~kg} \mathrm{~m}^{-3}$, Descamps et al. 2007), having a nominal density ratio of 1:2.2.

A collisionally disrupted mantle will create a swarm of icy blocks, containing little to no silicates or sulfides. If the slushy ice trapped enough organics to make the albedo low the individual blocks may have densities of $600 \lesssim \rho \lesssim 1100 \mathrm{~kg} \mathrm{~m}^{-3}$ depending on the degree of fracturing. If they re-assemble into a collisional rubble pile with $40 \%$ macroporosity their bulk densities are $360 \lesssim \rho \lesssim 660 \mathrm{~kg} \mathrm{~m}^{-3}$. Such objects may also have leaked from the EKB into the SD (Duncan et al. 1995; Levison \& Duncan 1997) and further into the Centaur population. The red points in Fig. 10 shows the densities of hot classicals (47171) $1999 \mathrm{TC}_{36}$ (Benecchi et al. 2010; Mommert et al. 2012), (26308) 1998 SM $_{165}$ (Spencer et al. 2006), and $2001 \mathrm{QC}_{298}$ (Vilenius et al. 2014), SD object (65489) Ceto, and Centaur (42355) Typhon (Santos-Sanz et al. 2012). Admittedly, most objects plot between the blue curves and could be thermally unaltered high- $\psi$ objects with a significant rock content. But they could also be rather compact objects containing little to no rock. In essence, cold and hot classicals may have similar bulk densities but for very different reasons - the former being porous and ice-poor, the latter being more compact and ice-rich. The object $2002 \mathrm{UX}_{25}$ is an example of a hot classical EKB that certainly is compact $(\psi \lesssim 20 \%)$ because of its large size $(D=650 \mathrm{~km})$ and must consist almost entirely of water ice due to its low density $\rho=820 \pm 110 \mathrm{~kg} \mathrm{~m}^{-3}$ (Brown 2013). This object could have formed in a collision between two much larger differentiated parents. This type of collision would be substantially less common in the primordial disk than in the EKB.

If comets formed in a collisional cascade their cumulative size distribution index $\alpha_{\mathrm{c}}$ should have a particular value. Theoretical work that assumes size-independent strength indicates that $\alpha_{\mathrm{c}}=2.51 \pm 0.01$ at equilibrium (Dohnanyi 1969; Tanaka et al. 1996). However, laboratory experiments and collision modeling (e.g., Benz \& Asphaug 1999) show that strength decreases with $D$ in the strength regime $(D \lesssim 0.5 \mathrm{~km})$ but increases with $D$ in the gravity regime $(D \gtrsim 0.5 \mathrm{~km})$. When accounting for this and when considering parameters for ice, solid basalt, or porous pumice (Benz \& Asphaug 1999; Jutzi et al. 2010) work by O'Brien \& Greenberg (2003) shows that $2.04 \leq \alpha_{\mathrm{c}} \leq 2.09$ in the gravity regime. This may steepen to $2.11 \leq \alpha_{\mathrm{c}} \leq 2.26$ when allowing for a distribution of collision velocities (Pan \& Schlichting 2012). These values can be compared to the empirical $\alpha_{\mathrm{c}}$ of small main belt asteroids, considered to be near steady state (Bottke et al. 2005): $\alpha_{\mathrm{c}}=3.00 \pm 0.05$ for $5 \lesssim D \lesssim 40 \mathrm{~km}$ (Ivezić et al. 2001), $\alpha_{\mathrm{c}}=2.55$ for $4 \lesssim D \lesssim 27 \mathrm{~km}$ if assuming $p_{\mathrm{V}}=0.1$ (Gladman et al. 2009), and $\alpha_{\mathrm{c}}=2.34 \pm 0.05$ for $2 \lesssim D \lesssim 25 \mathrm{~km}$ (Ryan et al. 2015).
These slopes tend to be steeper than modern theoretical predictions, suggesting that $\alpha_{\mathrm{c}}=2.7 \pm 0.4$ in the gravity regime.

Empirical size distributions for comets can be obtained in two ways - directly by measuring diameters of active or dormant comets and indirectly by considering jovian satellite craters. Direct measurements fall into two categories. J. Fernández et al. (1999) obtained a steep slope of $\alpha_{\mathrm{c}}=2.65 \pm 0.25$, later adjusted to $\alpha_{\mathrm{c}}=2.7 \pm 0.3$ by Tancredi et al. (2006). Others obtained shallower slopes, for example $\alpha_{\mathrm{c}}=1.6 \pm 0.1$ (Lowry et al. 2003), $\alpha_{\mathrm{c}}=1.45 \pm 0.05$ at $D=2-20 \mathrm{~km}$ and $\alpha_{\mathrm{c}}=1.91 \pm 0.06$ at $D=4-$ $10 \mathrm{~km}$ (Meech et al. 2004), $\alpha_{\mathrm{c}}=1.9 \pm 0.3$ (Lamy et al. 2004), $\alpha_{\mathrm{c}}=1.92 \pm 0.2$ (Snodgrass et al. 2011), and $\alpha_{\mathrm{c}}=1.92 \pm 0.23$ by Y. Fernández et al. (2013). Tancredi et al. (2006) suggest that too shallow slopes are obtained when a few large nuclei with uncertain sizes and albedos have an unproportional influence on the slope determination. Lamy et al. (2004) and Snodgrass et al. (2011) suggest that the steep slope of Fernández et al. (1999) is obtained by considering too few objects in a narrow size range, $D=4.2-6.6 \mathrm{~km}$. Tancredi et al. (2006) extended the range to $D=3.4-9 \mathrm{~km}$ but their steep slope may still be due to a too small upper diameter, according to Table 9 in Fernández et al. (2013). Fernández et al. (2013) may have overcome several of these problems by; (1) only using radiometrically determined $D$ and $p_{\mathrm{V}}$ based on Spitzer observations; (2) minimizing coma affects by only observing nuclei at $r_{\mathrm{h}} \geq 4 \mathrm{AU}$; (3) basing their slope calculation on the largest sample of comets considered to date (98 nuclei). Therefore, we adopt $\alpha_{\mathrm{c}}^{\mathrm{JFC}}=1.92 \pm 0.23$ (Fernández et al. 2013) for JFCs on average. Whitman et al. (2006) obtained $\alpha_{\mathrm{c}}=1.5 \pm 0.3$ for $\sim 60$ suspected dormant comets. Crater statistics on Ganymede yields $\alpha_{\mathrm{c}}=1.7$ for impactors with $2 \leq D \leq 5 \mathrm{~km}$ (Zahnle et al. 2003). These estimates are consistent with $\alpha_{\mathrm{c}}^{\mathrm{JFC}}$. If the effect of nucleus sublimation is accounted for, the $\alpha_{\mathrm{c}}^{\mathrm{JFC}}$-value may increase by $\sim 0.1$ (Weissman $\&$ Lowry 2003). Thus, the original size distribution of comets (if taken as $\alpha_{\mathrm{c}}^{\mathrm{JFC}} \approx 2.0$ ) may have been shallower than for similarlysized main belt asteroids.

For the strength regime theoretical work suggests a steep slope, $2.66 \leq \alpha_{\mathrm{c}} \leq 2.82$ (Benz \& Asphaug 1999; O’Brien \& Greenberg 2003; Jutzi et al. 2010; Pan \& Schlichting 2012). Thus, at collisional steady state we expect a higher number of small bodies compared to the extrapolated power law that fits the larger bodies. However, observations of main belt asteroids shows the opposite: $\alpha_{\mathrm{c}}=1.40 \pm 0.05$ (bluish spectrum) and $\alpha_{\mathrm{c}}=$ $1.20 \pm 0.05$ (reddish spectrum) for $0.4 \lesssim D \lesssim 5 \mathrm{~km}$ (Ivezić et al. 2001), $\alpha_{\mathrm{c}}=1.35 \pm 0.02$ (visual filter) and $\alpha_{\mathrm{c}}=1.79 \pm 0.07$ (red filter) for $D \lesssim 2.5 \mathrm{~km}$ (Wiegert et al. 2007), and $\alpha_{\mathrm{c}}=1.5 \pm 0.1$ for $D \lesssim 2.5 \mathrm{~km}$ (Gladman et al. 2009). The YORP spin-up and centrifugal disruption timescale is 400 times shorter than the collisional disruption timescale at $D=0.1 \mathrm{~km}$ (Denneau et al. 2015), and Jacobson et al. (2014) demonstrate that this makes the slope more shallow.

However, YORP is not effective at large $r_{\mathrm{h}}$, hence we expect numerous miniature comets if their reservoirs are collisionally evolved. This wealth of sub-km nuclei should be evident in the jovian satellite cratering record. Craters on Europa yield $\alpha_{\mathrm{c}}=0.9$ for $D \leq 1 \mathrm{~km}$ impactors while those on Ganymede yield $\alpha_{\mathrm{c}}=1.1 \pm 0.1$ at $D \leq 2 \mathrm{~km}$ (Zahnle et al. 2003). Fernández \& Morbidelli (2006) obtain $\alpha_{\mathrm{c}}=1.25 \pm 0.3$ for JFCs with $0.2 \lesssim D \lesssim 1 \mathrm{~km}$. Both teams note that the number of sub-km nuclei appears to be two orders of magnitude smaller than expected and a scarcity of sub-km nuclei has also been noted by others (Fernández et al. 1999; Meech et al. 2004; Fernández et al. 2013). Once comets reach $r_{\mathrm{h}} \lesssim 3$ AU sublimation torques can spin up nuclei far more efficiently than YORP. 
However, similarities between size distributions for active and dormant JFCs (Fernández \& Morbidelli 2006) may suggest that the frequency of rotational splitting does not have a strong sizedependence, as opposed to YORP. Zahnle et al. (2003) speculate that $99 \%$ of the $D=0.3 \mathrm{~km}$ nuclei might vanish before reaching Jupiter due to disruptive vaporization or "that small comets are intrinsically rare and that the Kuiper belt (or scattered disk) is not collisionally evolved". But if $D \lessgtr 1 \mathrm{~km}$ comets are common the small Uranian satellites are collisionally disrupted in $\lessgtr 0.5_{-0.4}^{+1.5} \mathrm{Gyr}$ (Zahnle et al. 2003) which casts further doubts on the existence of numerous miniature comets. If using the size distribution inferred from the jovian system, lifetimes grow to a more comfortable $\sim 2_{-1}^{+6}$ Gyr (Zahnle et al. 2003).

\subsection{Radiometric dating of comet material}

The Stardust samples from $81 \mathrm{P}$ offer a unique opportunity to perform radiometric dating of comet material. Matzel et al. (2010) place an upper limit ${ }^{26} \mathrm{Al} /{ }^{27} \mathrm{Al}<1 \times 10^{-5}$ on CAI particle Coki, meaning that its ${ }^{26} \mathrm{Al}-{ }^{26} \mathrm{Mg}$ systematics was reset $\geq 1.7 \mathrm{Myr}$ after CAI. Type C CAI like Coki (Sect. 3.1) often have little to no measurable ${ }^{26} \mathrm{Mg}$ excess (MacPherson et al. 1995) and have interacted with a ${ }^{16} \mathrm{O}$-poor gas that was not present during CAI formation but common during chondrule formation (Krot et al. 2007a). This in combination with Type B and C mineralogy led Krot et al. (2007b) to suggest that Type C CAI form when Type B CAI are being melted during the chondruleforming event. If correct, it means that Coki experienced melting and ${ }^{26} \mathrm{Al}-{ }^{26} \mathrm{Mg}$ chronology resetting $\sim 1.7 \mathrm{Myr}$ after it formed. Also Inti is lacking a measurable ${ }^{26} \mathrm{Al}$ excess, suggesting late re-setting (Ishii et al. 2010).

Ogliore et al. (2012) place an upper limit ${ }^{26} \mathrm{Al} /{ }^{27} \mathrm{Al}<$ $3 \times 10^{-6}$ on a chondrule fragment from $81 \mathrm{P}$ called Iris, meaning that its ${ }^{26} \mathrm{Al}-{ }^{26} \mathrm{Mg}$ systematics was reset $\geq 3 \mathrm{Myr}$ after CAI. Nakashima et al. (2015) place an upper limit ${ }^{26} \mathrm{Al} /{ }^{27} \mathrm{Al}<4 \times$ $10^{-6}$ on a chondrule fragment from 81P called Pyxie, meaning that its ${ }^{26} \mathrm{Al}-{ }^{26} \mathrm{Mg}$ systematics was reset $\geq 2.6 \mathrm{Myr}$ after CAI.

These measurements show that these particles were formed or processed in the inner solar system 1.7-3 Myr after CAI, and later were transported to the comet forming region. In fact, Ogliore et al. (2012) use their measurement to place a constraint on the formation time of Jupiter to $\geq 3 \mathrm{Myr}$ after CAI, because that event presumably would have terminated radial mixing across the snowline. The fact that these late-forming grains were baked into 81P (presumably at some significant depth, considering that a substantial layer may have been lost through sublimation) suggests that comet formation was a gradual process that extended over millions of years.

\subsection{Analysis}

Using the information presented in the previous sections we now discuss the possibility that comets are collisional rubble piles. The presence of a significant ${ }^{26} \mathrm{Al}$ abundance in the inner solar system at $t_{0}$ is well established. Such material was transported to the comet forming region at least during the first $\sim 3$ Myr years, if not present there already. Thus, the assumption of an ${ }^{26} \mathrm{Al}$ free outer solar system is a weak one. Astrophysical observations show that the major stellar accretion phase (Class 0 and I) lasts for a few times $10^{5} \mathrm{yr}$, thus a low-mass solar nebula forms early. Radiometric dating of some iron meteorites indicates their parent bodies may have formed in the inner solar system as early as a few $10^{5} \mathrm{yr}$ after CAI (Qin et al. 2008). Section 3.3 suggests that planetesimal formation took place in the outermost parts of the primordial disk when ${ }^{26} \mathrm{Al}$ was still present. It is therefore reasonable to assume that most of the 15-30 AU region was ready to form planetesimals during the first $\sim 1 \mathrm{Myr}$ after CAI.

Planetesimal formation in pebble swarms formed by streaming instabilities has become an increasingly popular scenario, for example, because of the high percentage of ultrawide binaries in the cold classical EKB. The key features of this scenario are that bodies form large, early, and rapidly. If so, these bodies were born with significant ${ }^{26} \mathrm{Al}$ and should have experienced strong thermal processing. If born at $\lesssim 1 \mathrm{Myr}$ after CAI, small comets crystallize, while large comets and small TNOs liquefy (Sect. 3.2). They re-freeze with high density, a high abundance of phyllosilicates, and are bereft of supervolatiles except for a $\sim 1 \mathrm{~km}$ surface layer containing $\mathrm{CO}, \mathrm{CO}_{2}$, and water ice that perhaps is amorphous (Sect. 3.2). A thicker layer containing supervolatiles is only possible if they formed $\gtrsim 10 \mathrm{Myr}$ after CAI (Sect. 3.2), thus it would be necessary to abandon streaming instabilities as the formation mechanism of TNOs altogether. We consider that an unattractive scenario.

The high density of (66652) Borasisi, $\rho=2100_{-1200}^{+2600} \mathrm{~kg} \mathrm{~m}^{-3}$ (Vilenius et al. 2014), is possibly due to thermal processing. Bodies like Phoebe and Himalia, that are denser than expected (Sect. 3.3), and clearly have been aqueously altered (Sect. 3.4), live up to expectations if the scenario sketched above is correct. Even when thermal processing is restricted to the core, the crust experiences collisional compaction in most of the primordial disk (Sect. 3.5).

When such bodies are shattered in a collisional cascade comet-sized collisional rubble piles will form out of the debris. They will be dominated by dense dirty ice blocks, potentially mixed with smaller quantities of supervolatile-bearing ice from the thin previous crust, and rocky core material, mixed at a low macro-porosity. They will inherit an elevated density $\left(\rho_{\text {bulk }} \approx 600-1100 \mathrm{~kg} \mathrm{~m}^{-3}\right)$, low porosity $(\psi \approx 40 \%)$, and significant strength $(1-10 \mathrm{MPa})$ on size scales corresponding to individual fragments $(\lessgtr 0.1-1 \mathrm{~km})$, thus being capable of sustaining overhangs of similar size. They may contain large chunks with highly differing ice-to-rock mass ratios. They will have little to no amorphous water ice, $\mathrm{N}_{2}, \mathrm{CO}, \mathrm{CH}_{4}, \mathrm{CO}_{2}$, and $\mathrm{NH}_{3}$. They will be rich in secondary minerals and phyllosilicates. Highresolution imaging of (25143) Itokawa does not suggest that collisional rubble-pile formation leads to the creation of concentric layering. The size distribution of these bodies would reflect their collisionally dominated history. Giant planet satellite systems are highly collisionally evolved (Bottke et al. 2010) and may contain bodies of this type. In fact, small $(D \lesssim 10 \mathrm{~km})$ jovian irregular satellites have $\alpha_{\mathrm{c}} \approx 2.5$ (Jewitt \& Haghighipour 2007), similar to that of small main belt asteroids (Sect. 3.5). Furthermore, comet-sized satellites like Callirrhoe, Megaclite, and Themisto appear to have been aqueously altered.

The properties expected for small collisional rubble piles should be compared to those of comet 67P; (1) a low bulk density $\rho_{\text {bulk }}=535 \pm 35 \mathrm{~kg} \mathrm{~m}^{-3}$ and high porosity of $\sim 70 \%$; (2) largescale structural weakness $\left(S_{\mathrm{t}}<0.15 \mathrm{kPa}\right)$ and no km-sized overhangs; (3) high abundances of $\mathrm{CO}$ and $\mathrm{CO}_{2}$, and a measurable content of $\mathrm{Ar}$ and $\mathrm{N}_{2}$; (4) no convincing signs of in situ aqueous alteration in 67P (or in $81 \mathrm{P}$ ); 5) clear evidence of concentric surface stratification in 67P (and layering in 9P). Considering these discrepancies, we do not find support for the hypothesis that $67 \mathrm{P}$ is a collisional rubble pile. 
When observed from ground a collisional rubble pile sufficiently close to the Sun would probably stand out as being exceptionally low in $\mathrm{CO}$ and $\mathrm{CO}_{2}$ relative to water. The $\mathrm{CO} / \mathrm{H}_{2} \mathrm{O}$ ratio varies by two orders of magnitude among comets, while the $\mathrm{CO}_{2} / \mathrm{H}_{2} \mathrm{O}$ ratio varies by one order of magnitude (A'Hearn et al. 2012), potentially supporting the existence of such objects among the JFCs. However, Reach et al. (2013) list nine $\mathrm{CO}, \mathrm{CO}_{2}$-poor targets that include $19 \mathrm{P} /$ Borrelly, $81 \mathrm{P} /$ Wild 2, and 103P/Hartley 2. A collision rubble-pile nature of these objects is highly questionable due to the low densities $\rho_{\text {bulk }}=180$ $300 \mathrm{~kg} \mathrm{~m}^{-3}$ of 19P (Davidsson \& Gutiérrez 2004) and $\rho_{\text {bulk }}=$ 200-400 kg m ${ }^{-3}$ of 103P (Thomas et al. 2013) and apparent lack of in situ aqueous alteration in 81P. Thus, a low $\mathrm{CO}, \mathrm{CO}_{2-}$ abundances does not automatically indicate a collisional rubblepile origin.

Large comets like Hale-Bopp $(D=74 \pm 6 \mathrm{~km})$, 28P/Neujmin $1(D=22-30 \mathrm{~km}), 29 \mathrm{P} /$ SchwassmannWachmann $1(D=30 \mathrm{~km})$, and 109P/Swift-Tuttle $(D=24 \mathrm{~km})$ display a palette of behavior that is qualitatively similar to that of small comets. Even Hale-Bopp is rich in supervolatiles and may contain amorphous ice. The Giotto (Keller et al. 1988) and VEGA (Merényi et al. 1990) images of 1P/Halley $(D=11 \mathrm{~km})$ show a comet similar to the smaller JFCs visited by spacecraft and its bulk density is $\rho_{\text {bulk }}=500-1200 \mathrm{~kg} \mathrm{~m}^{-3}$ with a preference for the lower value (Rickman 1986; Skorov \& Rickman 1999), consistent with the estimate $\rho_{\text {bulk }}=600_{-400}^{+900} \mathrm{~kg} \mathrm{~m}^{-3}$ (Sagdeev et al. 1986), thus comparable to that of 67P. One cannot argue that small comets formed in collisions among such large comets - if collisions where important, large comets would be dense, deprived of supervolatiles and aqueously altered, like their small TNO parents. Those properties would be passed on to small comets. Furthermore, the size distribution of JFCs may not be consistent with collisional steady state due to an apparent two orders of magnitude deficit of sub-kilometer nuclei, and a potential discrepancy in slope for larger nuclei with respect to similar-sized main belt asteroids (Sect. 3.5).

Taken at face value, the high abundance of supervolatiles (A'Hearn et al. 2012), the low bulk densities (Sect. 2.1), and the structural weakness illustrated by nucleus splitting (Boehnhardt 2004), suggest that comet nuclei and collisional rubble piles constitute two distinct and unrelated populations.

Gounelle et al. (2008) suggest that grade 1 CI meteorites may have a comet origin based on the possibility that CI1 meteorite Orgueil may have had a JFC pre-impact orbit. They note that CI1 mineralogy is dominated by phyllosilicates, carbonates, and magnetite, while olivine and pyroxene are very rare. They suggest that comets have experienced extensive aqueous alteration due to short-lived radionuclides. Based on data available at the time they argued that $\mathrm{CI} 1 \mathrm{D} / \mathrm{H}$ ratios $(\$ 1.3$ times terrestrial) were consistent with those of comets to within error bars. However, Orgueil has a density of $\rho=2420 \mathrm{~kg} \mathrm{~m}^{-3}$ (Macke et al. 2011) and carbonaceous chondrites have tensile strengths $0.3 \leq S_{\mathrm{t}} \leq 30 \mathrm{MPa}$ (Tsuchiyama et al. 2009) - values that are much higher than those of comets. Cometary D/H ratios are often significantly higher than those of chondrites (Altwegg et al. 2015) and there is no convincing evidence of aqueous alteration in comets thus far. Therefore, comets are completely unsuitable parents of CI1 carbonaceous chondrites, while the collisional rubble piles would be ideal parents. We do not claim that CI carbonaceous chondrites stem from deep within the primordial disk but consider them excellent analogues for the material to be expected within small TNOs and their collisional fragments. They do not look anything like comets.
But if comets are not collisional rubble piles, how did they form? In Sect. 4 we propose a concurrent comet and TNO formation scenario and attempt to describe the properties of primordial rubble piles as expected from first principles. These are successfully compared with the properties of comet nuclei in Sect. 5.

\section{A comet formation scenario}

We here describe a formation scenario of comets and TNOs. We discuss initial conditions (Sect. 4.1), the formation of mediumsized TNOs (Sect. 4.2), and cometesimals (Sect. 4.3) in the solar nebula. Sect. 4.4 describes the concurrent evolution of such bodies into comets and large TNOs in the primordial disk, focusing on the comet size distribution in Sect. 4.4.1 and debris production during TNO collisions in Sect. 4.4.2. Section 4.5 describes the transport of these bodies to the EKB, SD, and OC.

\subsection{Conditions at $15-30$ AU from the Sun}

We assume that the $r_{\mathrm{h}}=15-30 \mathrm{AU}$ region had a mass $M_{\mathrm{PD}}=$ $15 M_{\oplus}$ of solids. This is the lowest mass considered consistent with the Nice model (Alessandro Morbidelli, priv. comm.). We focus on this low-mass case because the survival of primordial comet nuclei against collisions cannot be guaranteed for higher disk masses (Morbidelli \& Rickman 2015; Rickman et al. 2015). Our $M_{\mathrm{PD}}$ is consistent with the work of Nesvorný et al. (2013), who showed that a disk mass of $14-28 M_{\oplus}$ is necessary to explain the number of Jupiter Trojans.

We now discuss the conditions under which $M_{\mathrm{PD}}=15 M_{\oplus}$ is consistent with the size of the EKB. By utilizing empirical size distributions combined with an assumed density of $1000 \mathrm{~kg} \mathrm{~m}^{-3}$, Fraser et al. (2014) estimated a mass $0.01 M_{\oplus}$ for the hot classical EKB and $3 \times 10^{-4} M_{\oplus}$ for the cold classical EKB. If the average density is taken as $2500 \mathrm{~kg} \mathrm{~m}^{-3}$ the total current mass may be as high as $M_{\mathrm{EKB}}=0.026 M_{\oplus}$. The EKB has a fractional loss rate of $3 \times 10^{-11} \mathrm{yr}^{-1}$ (Duncan et al. 1995), translating to a $\sim 12 \%$ reduction of the original population in the last $4 \mathrm{Gyr}$. Combined with $M_{\mathrm{PD}}=15 M_{\oplus}$ this implies a transfer efficiency $P_{\mathrm{EKB}}=$ $0.19 \%$ from the primordial disk to the EKB.

The theoretical value of $P_{\mathrm{EKB}}$ based on numerical modeling of ice giant migration through the primordial disk is still under debate. Levison et al. $(2008,2011)$ obtain $P_{\mathrm{EKB}}=0.3 \%-0.6 \%$ by assuming a high initial eccentricity $e_{0}=0.3$ for Neptune, a short migration (from $r_{\mathrm{h}}=27.5-28.9 \mathrm{AU}$ to $30.1 \mathrm{AU}$ ), and rapid $e$-damping on a time scale of $\tau \approx 1 \mathrm{Myr}$. But according to Nesvorný (2015) this rapid migration does not reproduce EKB inclinations and to resolve this problem he suggested $e_{0}=0$, a longer migration distance from $r_{\mathrm{h}}=24 \mathrm{AU}$ to $30.1 \mathrm{AU}$, and slower damping $(\tau=10-30 \mathrm{Myr})$. This leads to $P_{\mathrm{EKB}}=0.02 \%-$ $0.04 \%$. Nevertheless, Nesvorný (2015) noted that this solution suffers from the "resonance overpopulation problem": the $3: 2$ MMR population is 5-10 times larger than observed. He also noted that the usage of $\tau=100 \mathrm{Myr}$ substantially reduced the problem and that such a slow damping "may require a very low mass of the planetesimal disk", which is consistent with our current assumption. For this scenario Nesvorný (2015) obtained $P_{\text {EKB }}=0.32 \%$, similar to that of Levison et al. $(2008,2011)$. Therefore, we consider $P_{\mathrm{EKB}}=0.19 \%$ a reasonable value. 
Assuming that the surface density $\Sigma\left[\mathrm{g} \mathrm{cm}^{-2}\right]$ is inversely proportional to distance, a $15 M_{\oplus}$ mass distributed between 15$30 \mathrm{AU}$ is obtained for

$\Sigma=4.23 r_{\mathrm{h}}^{-1}$

that yields $\Sigma=0.282 \mathrm{~g} \mathrm{~cm}^{-2}$ at $15 \mathrm{AU}$ and $\Sigma=0.142 \mathrm{~g} \mathrm{~cm}^{-2}$ at $30 \mathrm{AU}$. For comparison, our surface density at $r_{\mathrm{h}}=30 \mathrm{AU}$ is a factor of 1.29 below that of the minimum mass solar nebula (Hayashi 1981).

\subsection{Formation of medium-sized TNOs}

Weidenschilling (1997) modeled the hierarchical agglomeration of initially $\mu \mathrm{m}$-sized monomer grains at $30 \mathrm{AU}$ assuming $\Sigma=$ $0.41 \mathrm{~g} \mathrm{~cm}^{-2}$. We use this simulation (assumed to start at $t_{0}$ ) as a guide-line but apply a 1.5-2.9 times slower growth rate because of our lower $\Sigma$. Relative velocities of $v_{\text {rel }} \sim 1 \mathrm{~mm} \mathrm{~s}^{-1}$ due to Brownian motion leads to thermal coagulation and growth of $\sim 0.1 \mathrm{~mm}$ fractal clusters in a few $10^{4} \mathrm{yr}$. Gas turbulence then replaces Brownian motion as the source of relative velocity, leading to growth of $\sim 1 \mathrm{~mm}$ clusters at $v_{\text {rel }} \sim 0.1 \mathrm{~m} \mathrm{~s}^{-1}$. At such a $v_{\text {rel }}$ the restructuring limit is passed and the clusters are no longer fractals though they are still very porous. Then vertical settling sets in and clusters fall to the midplane while growing to pebbles of $\mathrm{cm}$-size. Because of a pressure gradient gas orbits the Sun with a velocity that is $50 \pm 10 \mathrm{~m} \mathrm{~s}^{-1}$ lower than the Keplerian velocity $v_{\mathrm{K}}$ at 15-30 AU (Weidenschilling 1977). The concentration of pebbles to the midplane locally accelerates the gas to $v_{\mathrm{K}}$, creating a shear flow against the slower gas outside the midplane. Owing to the Kelvin-Helmholtz instability the gas becomes turbulent. This shear-induced turbulence prevents the layer of clusters and pebbles of becoming thinner than $h \sim 10^{-2} \mathrm{AU}$. This stage is reached at $t=0.08-0.15 \mathrm{Myr}$.

The turbulence of the midplane layer (potentially increased by magneto-rotational instabilities) introduces local pressure enhancements that concentrate $\mathrm{cm}$-sized pebbles into swarms that grow efficiently due to the streaming instability (Youdin \& Goodman 2005). The non-dimensional gravitational parameter

$$
\tilde{G}=\frac{4 \pi \Sigma r_{\mathrm{h}}^{3}}{h M_{\odot}}
$$

determines the maximum size of the pebble swarms (e.g., Johansen et al. 2012). With $h=10^{-2}$ AU we obtain $\tilde{G}=0.13$ at the inner part of the disk and $\tilde{G}=0.54$ at its outer part. The former value corresponds to $D \approx 900 \mathrm{~km}$ if collapsing to a single body (Johansen et al. 2012). The latter value is uncertain due to an assumed scaling law that indicates $D \approx 1400 \mathrm{~km}$. However, $\mathrm{N}$-body simulations of gravitational collapse of pebble swarms (Nesvorný et al. 2010) indicate that only a fraction of the swarm mass (determined by the angular momentum) is used for building TNO-sized bodies while the rest disperses. Furthermore, the swarms do not build a single large body but several smaller ones.

Guided by the measured sizes of cold classical disk objects that experienced limited post-formation accretion (Sect. 3.3.1) we assume $D \leq 400 \mathrm{~km}$ for newly formed TNOs. Wahlberg Jansson \& Johansen (2014) show that streaming instabilities also may form $D=1-10 \mathrm{~km}$ objects, albeit on time scales that are $\sim 100$ times longer than for $D \sim 100 \mathrm{~km}$ objects. It is questionable if the formation of such small bodies could proceed unperturbed. But even if this was the case it is plausible that small icy objects formed near $t_{0}$ would disintegrate by steam pressure (Sect. 3.2.2). Thus we place a lower limit of
$D \geq 50 \mathrm{~km}$, which is consistent with the "Missing Intermediatesized Planetesimal problem" discussed by Sheppard \& Trujillo (2010): Neptune Trojans (that once were primordial disk objects) with $32 \leq D \leq 90 \mathrm{~km}$ are at least $80 \pm 10$ times fewer than expected from the size distribution measured at larger sizes.

Swarms containing a sufficient amount of pebbles to build a TNO-sized body may form in less than $\sim 10$ orbits or just 600-1600 yr (Johansen et al. 2007). The gravitational collapse of a swarm takes $\sim 100 \mathrm{yr}$ (Nesvorný et al. 2010). Therefore, we expect that the components of TNO-sized binaries initially had $D \approx 50-400 \mathrm{~km}$ and that they formed during the first few $0.1 \mathrm{Myr}$ after CAI. We assume they form with the size distribution measured for cold classical disk objects, that is to say, a broken power-law with differential index $q=8.2 \pm 1.5$ at $D \geq 140 \mathrm{~km}$ and $q=2.9 \pm 0.3$ below that size (Fraser et al. 2014).

It is not likely that streaming instabilities managed to consume all pebbles in the disk. To illustrate this point we apply a $1.4 \%$ dust-to-gas mass ratio (Lodders 2003), resulting in a gas surface density $\Sigma_{\text {gas }}=10.0 \mathrm{~g} \mathrm{~cm}^{-2}$ at $r_{\mathrm{h}}=30 \mathrm{AU}$. A midplane gas mass density of $\rho_{\text {gas }}(0)=1.3 \times 10^{-13} \mathrm{~g} \mathrm{~cm}^{-3}$ is obtained if using the vertical profile

$\rho_{\text {gas }}(z)=\rho_{\text {gas }}(0) \exp \left(-\frac{z^{2}}{H^{2}}\right)$

where $z$ is the height above the midplane and the gas scale height is taken as $H=3 \mathrm{AU}$. Using the previous values of $h$ and $\Sigma$ for the dust the midplane dust density is $\rho_{\text {dust }}=9.5 \times 10^{-13} \mathrm{~g} \mathrm{~cm}^{-3}$, thus vertical settling alone has created a mass loading (local dust-to-gas mass ratio) of 7.5. However, to enable gravitational collapse of pebbles their mass density must be higher than the Roche density (e.g., Johansen et al. 2014),

$\rho_{\mathrm{R}}=\frac{9 M_{\odot}}{4 \pi r_{\mathrm{h}}^{3}}$

where $M_{\odot}$ is the solar mass. Here it is $\rho_{\mathrm{R}}=1.58 \times 10^{-11} \mathrm{~g} \mathrm{~cm}^{-3}$, therefore $\rho_{\text {dust }}$ is a factor of $\sim 17$ too low for gravitational collapse. Streaming instabilities elevate $\rho_{\text {dust }}$ further and according to Johansen et al. (2014) the pebble swarms may reach $\rho_{\text {dust }} \approx 1000 \rho_{\text {gas }}(0) \approx 1.3 \times 10^{-10} \mathrm{~g} \mathrm{~cm}^{-2}$ when the nominal mass loading is at least 3 , which is the case here. This value of the pebble density is 8.0 times $\rho_{\mathrm{R}}$. This shows that formation of TNO-sized bodies indeed is expected under these conditions but also that this mode of planetesimal growth will grind to a halt when the density of free pebbles has fallen by a factor of $\sim 8$. In other words, the formation of TNO-sized bodies can only proceed until $\sim 87 \%$ of the pebbles have been consumed, then it stops with $\sim 13 \%$ of the pebbles still remaining in the solar nebula. An evaluation made at $r_{\mathrm{h}}=15 \mathrm{AU}$ tells a similar story although the fraction of surviving pebbles may approach $25 \%$ (if taking the scale height there as $H=1.5 \mathrm{AU}$ ). Considering the uncertainties involved we apply a flat $13 \%$ survival probability of pebbles against TNO formation throughout the primordial disk. Specifically, the surface density of pebbles is now

$\Sigma_{\mathrm{p}}=0.55 r_{\mathrm{h}}^{-1}$

while the surface density of TNOs is

$\Sigma_{\mathrm{TNO}}=3.68 r_{\mathrm{h}}^{-1}$.

Thus, $\Sigma_{\mathrm{p}}=0.037 \mathrm{~g} \mathrm{~cm}^{-2}$ at $15 \mathrm{AU}$ and $\Sigma_{\mathrm{p}}=0.018 \mathrm{~g} \mathrm{~cm}^{-2}$ at $30 \mathrm{AU}$. The TNO-sized bodies constitute $13 M_{\oplus}$ in total and there 
is still $2 M_{\oplus}$ of pebbles left in the primordial disk. With the applied power law (here using $0.5 \mathrm{~km}$-wide diameter bins) there are 17 bodies in the bin with $D=400 \mathrm{~km}, 1.8 \times 10^{6}$ bodies in the bin with $D=50 \mathrm{~km}$, and $8.5 \times 10^{7}$ TNOs in total. We note that this model has $3.0 \times 10^{7}$ TNOs with $D \geq 80 \mathrm{~km}$, consistent with the $(3-4) \times 10^{7}$ bodies of this size needed to populate the Jupiter Trojan swarms (Nesvorný et al. 2013).

About $\sim 350$ bodies have $D \geq 391 \mathrm{~km}$, corresponding to a mass of at least $90 \%$ of that of the largest ones. We propose that these large bodies experienced $\sim 0.4 \mathrm{Gyr}$ of runaway growth, primarily by consuming smaller TNOs, and reached the sizes of Triton $(D=2706 \pm 2 \mathrm{~km})$, Pluto, and Eris $(D \approx 2300 \mathrm{~km})$ just prior to the giant planet instability that disrupted the primordial disk. Before exploring this growth in more detail we turn our attention to the $2 M_{\oplus}$ of pebbles that survived the early and short TNO-formation phase.

\subsection{Formation of cometesimals}

Planetesimal formation has often been described as a choice between hierarchical agglomeration and streaming instabilities. We explicitly propose a hybrid model where both growth scenarios play important roles, but at different size ranges. We thus return to the Weidenschilling (1997) scenario, assuming only two modification with respect to his work; (1) the timescale of growth is increased by factors of 11.1 at $15 \mathrm{AU}$ and of 22.8 at $30 \mathrm{AU}$ according to Eq. (6); (2) the TNO-sized bodies present at the onset of hierarchical agglomeration (not considered in Weidenschilling's work) efficiently consume $D \lesssim 1 \mathrm{~km}$ objects due to gravitational focusing. We assume that $v_{\text {rel-values }}$ among bodies built by hierarchical agglomeration are unaltered by the presence of TNO-sized bodies, except in their immediate vicinity, because gravitational perturbations on small bodies are quickly damped by gas drag in the solar nebula. We assume that the average growth rate is unaffected by the presence of highdensity swarms, since their accelerated hierarchical agglomeration is compensated for by slower growth in low-density regions between swarms.

The key feature of hierarchical growth is that particles of different size have different stopping times (the time it takes for a velocity difference with respect to the gas to be damped by gas drag). Larger particles (with longer stopping times) need more time to adjust to changes in the turbulent gas environment than smaller particles, thus non-zero relative velocities are introduced between small and large grains. This is a necessary condition for collisions, thus possibility of growth. Similar-sized particles have small relative velocities (because they respond similarly to changes in the gas flow properties). Therefore, another signature feature of hierarchical growth is that large particles grow by accreting much smaller particles and not similar-sized ones. The magnitude of $v_{\text {rel }}$ is essentially determined by the sub-Keplerian motion of the gas. Particles with $D \ll 1 \mathrm{~m}$ have short stopping times, thus move with the same sub-Keplerian transverse velocity as the gas and display no radial drift. Particles with $D \approx 0.01-1 \mathrm{~m}$ gradually develop a radial drift toward the Sun of at most a few $10 \mathrm{~m} \mathrm{~s}^{-1}$ due to lack of pressure support. Their transverse velocities approach $v_{\mathrm{K}}$. Particles with $D \gg 1 \mathrm{~m}$ become increasingly decoupled from the gas, and essentially move on Keplerian orbits with no radial drift. The detailed dynamical model of Weidenschilling (1997) shows what kind of projectiles and targets that collide, how often this takes place, and at what relative velocity.

The pebble size distribution in the midplane is initially bimodal (Weidenschilling 1997). The two peaks consist of locally grown clusters with $D \approx 0.1 \mathrm{~mm}$, mixed with $\mathrm{cm}$-sized pebbles grown during vertical settling from $|z| \gg 0$ to the midplane. The largest pebbles have $D \approx 0.1 \mathrm{~m}$ and gain most of their mass by accreting $30-50 \mu \mathrm{m}$ clusters at $v_{\text {rel }}=10 \mathrm{~m} \mathrm{~s}^{-1}$. Such growth has recently been discussed in the context of the so-called bouncing barrier. Weidenschilling (1997) did not consider bouncing of aggregates as a possible collisional outcome, which was however observed in many laboratory experiments (Blum \& Wurm 2008; Güttler et al. 2010) and shown to be relevant under realistic conditions (Zsom et al. 2010). This typically prohibits growth into $0.1 \mathrm{~m}$ aggregates. Windmark et al. (2012a,b) have opened a possibility to overcome this bouncing barrier through sufficient size differences and relative velocities of colliding aggregates. They achieve this by either introducing very few centimeter particles as seeds (Windmark et al. 2012a), or allowing for a significant velocity dispersion (Windmark et al. 2012b). Some "lucky winners" in these collisions can then grow sufficiently to overcome the bouncing barrier (Windmark et al. 2012b). We conclude that the growth into decimeter-sized particles is currently accepted even when taking the complexity of laboratory experiments into account and it produces a similar distribution as the one considered by Weidenschilling (1997).

The $D \approx 0.1 \mathrm{~m}$ aggregates have overcome the bouncing barrier but experienced many bouncing collisions, thus an average porosity of $\psi \approx 70 \%$ is expected (Weidling et al. 2009) and an even denser surface. According to Windmark et al. (2012a) these large aggregates can grow from smaller ones if the collisions are violent enough to fragment the small ones but gentle enough not to erode the large ones. The velocity range from their Fig. 7 (top right) is $0.03-3.0 \mathrm{~m} \mathrm{~s}^{-1}$ but this is based on experiments with silicate material. To account for ice and organics present beyond the snow line we must recognize that the surface energy is significantly larger for these materials. The experiments by Kouchi et al. (2002) and Kudo et al. (2002) on organics and by Gundlach $\&$ Blum (2015) on water ice indeed show that both substances are significantly stickier than silica. It is thus reasonable to assume that the velocity range for growth as described above can be an order of magnitude higher, or $0.3 \lesssim v_{\text {rel }} \lesssim 30 \mathrm{~m} \mathrm{~s}^{-1}$ (Wada et al. 2009).

Formation of meter-sized boulders is therefore expected since their sweep-up of $1-10 \mathrm{~cm}$ targets is expected to take place at $v_{\text {rel }} \lesssim 30 \mathrm{~m} \mathrm{~s}^{-1}$. According to Weidenschilling (1997), with our modified timescale, meter-sized boulders have formed at $t=0.28-0.56 \mathrm{Myr}$ after CAI. The size distribution has now lost its bimodal shape and turned into a power-law because fragmentation re-populates mass bins at all levels (Weidenschilling 1997). At these velocities, composition A material is compressed to $\psi \approx 60 \%$ or a density $\rho \approx 730 \mathrm{~kg} \mathrm{~m}^{-3}$ (composition B has $\psi \approx 72 \%$ and $\rho \approx 350 \mathrm{~kg} \mathrm{~m}^{-3}$ ).

But differences in stopping times are smaller the larger are the collision partners, thus $v_{\text {rel }}$ decreases. This means that the maximum level of compression and strength are reached for bodies being a few meters in size. For example, once the maximum diameter has reached $D=6.7 \mathrm{~m}$ and the median value is $\langle D\rangle=$ $1.3 \mathrm{~m}$ the largest bodies grow by accreting $D=2-3 \mathrm{~m}$ bodies at a relative velocity as low as $v_{\text {rel }}=2 \mathrm{~m} \mathrm{~s}^{-1}$ (Weidenschilling 1997).

The largest threat to meter-sized boulders in classical models is the drift barrier (e.g., Weidenschilling 1977), that is, their radial motion is sufficiently rapid to cause large-scale accretion of solid material by the protosun which may prevent the formation of planetary systems. In this context we note that the pressure bumps responsible for creating collapsing swarms in the streaming-instability scenario still are present. They no longer are capable of concentrating material to the point of gravitational 
collapse but they may still affect the dynamics of small boulders. The pressure bumps that ultimately were responsible for the growth of TNO-sized bodies may therefore also assist the hierarchical growth of cometesimals by reducing their radial drift.

At $t=0.41-0.83 \mathrm{Myr}$ the largest bodies have $D=70 \mathrm{~m}$ and they feed on $D=20 \mathrm{~m}$ bodies that completely dominate the size distribution. They collide at $v_{\text {rel }}=0.8 \mathrm{~m} \mathrm{~s}^{-1}$. There is a pronounced lack of $D=1-5 \mathrm{~m}$ bodies because they have been consumed to form the $D=20 \mathrm{~m}$ bodies. At $t=1.19-2.43 \mathrm{Myr}$ essentially all the mass is locked up in $D=100 \mathrm{~m}$ bodies. The largest bodies have $D=500 \mathrm{~m}$ and they grow by consuming $D=200 \mathrm{~m}$ bodies at $v_{\text {rel }}=0.2 \mathrm{~m} \mathrm{~s}^{-1}$.

At $t=1.74-3.57 \mathrm{Myr}$ after CAI the largest bodies have $D=6 \mathrm{~km}$ and they primarily feed on $D=1 \mathrm{~km}$ bodies at $v_{\text {rel }}=2 \mathrm{~m} \mathrm{~s}^{-1}$. The relative velocity is an order of magnitude higher than among the $D=100 \mathrm{~m}-500 \mathrm{~m}$ bodies because gravitational attraction between collision partners starts to play an increasingly important role. The size range of cometesimals at this stage is limited to $100 \lessgtr D \lessgtr 6000 \mathrm{~m}$. There is a strong depletion of objects in the $1-100 \mathrm{~m}$ range. There is plenty of debris (by number) with sizes in the $1 \mu \mathrm{m}-1 \mathrm{~m}$ range (Weidenschilling 1997).

In the $D=1 \mu \mathrm{m}-2.8 \mathrm{~km}$ range (ignoring the gap at $D \approx$ $0.5 \mathrm{~m}-0.1 \mathrm{~km}$ ) the incremental size distribution (Fig. 11 from Weidenschilling 1997) is well fit with a power-law with an in$\operatorname{dex} \beta=2.48$. Considering the bin sizes used by Weidenschilling (1997) this corresponds to a differential size distribution slope of $q=3.47$. We note that the mass in $D<1 \mathrm{~m}$ debris is only $0.02 M_{\oplus}$ and can be ignored. The total number of cometesimals is $\sim 2.08 \times 10^{15}$ with $0.34 M_{\oplus}\left(2.04 \times 10^{15}\right.$ nuclei $)$ at $0.1 \leq D \leq 0.5 \mathrm{~km}, 0.65 M_{\oplus}\left(3.78 \times 10^{13}\right.$ nuclei $)$ at $0.5 \leq$ $D \leq 2 \mathrm{~km}, 0.55 M_{\oplus}\left(1.04 \times 10^{12}\right.$ nuclei $)$ at $2 \leq D \leq 4 \mathrm{~km}$, and $0.43 M_{\oplus}\left(1.45 \times 10^{11}\right.$ nuclei $)$ at $4 \leq D \leq 6 \mathrm{~km}$. A bulk density of $\rho_{\text {bulk }}=300 \mathrm{~kg} \mathrm{~m}^{-3}$ has been assumed.

The number density of TNO-sized bodies is too low to efficiently accrete the cometesimals on a $\sim 1$ Myr time scale, with one exception. In case the relative velocity between cometesimals and the dynamically cold TNOs drops below $v_{\text {rel }} \lesssim$ $0.5 \mathrm{~m} \mathrm{~s}^{-1}$ the gravitational focusing of the TNOs (enhancement of their physical cross section by virtue of their gravitational force) becomes so efficient that it overcompensates for their low number density. For this to take place the cometesimals need to be nearly insensitive to gas drag. A radial drift speed of $\lesssim 0.5 \mathrm{~m} \mathrm{~s}^{-1}$ requires $D \gtrsim 100 \mathrm{~m}$ while $\lesssim 0.01 \mathrm{~m} \mathrm{~s}^{-1}$ requires $D \gtrsim 1 \mathrm{~km}$. However, the decoupling from the gas also means that $D \gtrsim 1 \mathrm{~km}$ cometesimals become viscously stirred by the TNOs which may increase $v_{\text {rel }}$ to the point that gravitational focusing becomes inefficient. We therefore expect $0.1 \lesssim D \lesssim 1 \mathrm{~km}$ cometesimals to become strongly depleted. If the relative velocities between cometesimals and TNOs are $0.1 \mathrm{~m} \mathrm{~s}^{-1}$ or $0.2 \mathrm{~m} \mathrm{~s}^{-1}$ the number of cometesimals will drop by factors of 204 or 14 during a 2 Myr time period (according to calculations resembling those with Eq. (10) in Sect. 4.4.1). If the relative velocities are $0.3 \mathrm{~m} \mathrm{~s}^{-1}$ or $0.5 \mathrm{~m} \mathrm{~s}^{-1}$ the number of cometesimals drops by a factor of 5.9 or 2.9. This shows that the effect is important but highly selective. It is beyond the scope of this paper to determine through numerical modeling how this accretion alters the size distribution with respect to that of Weidenschilling (1997) at $t=1.74-3.57 \mathrm{Myr}$. We here keep the size distribution $(q=3.47)$ and absolute numbers presented above for $D \geq 1 \mathrm{~km}$ but apply a flat distribution at smaller sizes. This reduces the number of $D \leq 1 \mathrm{~km}$ cometesimals by a factor of $\sim 170$ and transfers $0.51 M_{\oplus}$ from the cometesimal population to the TNOs.
We have now reached a critical point in the evolutionary history of the solar nebula. We assume that the gas disperses toward the end of the $t=1.74-3.57 \mathrm{Myr}$ interval after CAI (Sect. 3.2.4). It means that the rules of the game change and that further evolution of cometesimals and TNO-sized bodies should be studied in a gas-free environment. At this breaking-point in early solar system history we find that the cometesimals have the following properties.

1. The cometesimals are single-lobed because they systematically have grown by accreting bodies 3-6 times smaller than themselves (a $D=2 \mathrm{~km}$ body has $0.3-0.7 \mathrm{~km}$ substructure). These subunits could have lived and aged for $\sim 10^{5} \mathrm{yr}$ prior to accretion.

2. The cometesimals contain dense $\left(350 \lesssim \rho \lesssim 730 \mathrm{~kg} \mathrm{~m}^{-3}\right)$ meter-sized units with $\psi \gtrsim 60 \%$ micro-porosity. If such boulders form bunches with $40 \%$ macro-porosity a cometesimal bulk density of $210 \lesssim \varrho_{\text {bulk }} \lesssim 440 \mathrm{~kg} \mathrm{~m}^{-3}$ is expected.

3. The km-sized bodies contain 1-100 $\mathrm{m}$ cavities.

4. The differential size distribution above $D \approx 1 \mathrm{~km}$ has a power law index of $q \approx 3.5$ but there is a substantial depletion of cometesimals below that size.

\subsection{Concurrent cometesimal and TNO evolution}

The gas-free primordial disk existed for another $\sim 0.4 \mathrm{Gyr}$ before being disrupted by Uranus and Neptune. During this time a number of processes took place. Trans-Neptunian objects grew by consuming each other and cometesimals, small TNOs and cometesimals experienced viscous stirring (increasing their $e, i$, and $v_{\text {rel }}$ ), while the dynamical heating of large TNOs was damped by dynamical friction against the smaller bodies.

We use the two-groups approximation (Eqs. (59)-(61) in Goldreich et al. 2004) to model the runaway growth of the largest TNOs and the effects of viscous stirring and dynamical friction. The disk population is divided into large and small objects. The large objects with random velocity $v$ are taken as the 350 largest TNOs (Sect. 4.2). The small objects are all other TNOs plus the comets, having random velocity $u$. The diameters, masses, and escape velocities of large bodies are time-dependent, as well as $u$ and $v$. The solutions at $r_{\mathrm{h}}=15 \mathrm{AU}$ and $r_{\mathrm{h}}=30 \mathrm{AU}$ are seen in Fig. 12. At $t=400 \mathrm{Myr}$ the largest bodies have $1.43 D_{\mathrm{T}}$ and $2.9 M_{\mathrm{T}}$ at $r_{\mathrm{h}}=15 \mathrm{AU}$ but $1.20 D_{\mathrm{T}}$ and $1.7 M_{\mathrm{T}}$ at $r_{\mathrm{h}}=30 \mathrm{AU}\left(D_{\mathrm{T}}\right.$ and $M_{\mathrm{T}}$ being the diameter and mass of Triton).

The random velocities of small TNOs and comets increase due to viscous stirring. At $r_{\mathrm{h}}=30 \mathrm{AU}$ we have $u<100 \mathrm{~m} \mathrm{~s}^{-1}$ during the first $\sim 88 \mathrm{Myr}$ and $u=282 \mathrm{~m} \mathrm{~s}^{-1}$ at $t=400 \mathrm{Myr}$. At $r_{\mathrm{h}}=15 \mathrm{AU}$ the random velocity approaches $u=740 \mathrm{~m} \mathrm{~s}^{-1}$ at the end of the simulation but remains at $u<200 \mathrm{~m} \mathrm{~s}^{-1}$ during the first $\sim 54 \mathrm{Myr}$ and at $u<100 \mathrm{~m} \mathrm{~s}^{-1}$ during the first $\sim 20 \mathrm{Myr}$. The random velocity $v$ of the largest bodies is smaller due to dynamical friction.

Numerical runaway growth models indicate (Kokubo \& Ida 1996; Weidenschilling et al. 1997) that

$$
\left\{\begin{array}{l}
u=v_{\mathrm{K}} \sqrt{e^{2}+\sin ^{2} i} \\
\sin i \approx 0.6 e .
\end{array}\right.
$$

We here use Eq. (8) and $u(t)$ from Fig. 12 to calculate $i$ and $e$ of small bodies. As can be seen in Fig. 13 the viscous stirring is puffing up the layer of small bodies by increasing the inclination to $i \approx 3^{\circ}$ at $r_{\mathrm{h}}=15 \mathrm{AU}$ and $i \approx 1^{\circ}$ at $r_{\mathrm{h}}=30 \mathrm{AU}$. The disk thickness increases from $h \approx 0.01 \mathrm{AU}$ to $h=1.48 \mathrm{AU}$ at $r_{\mathrm{h}}=15 \mathrm{AU}$ 

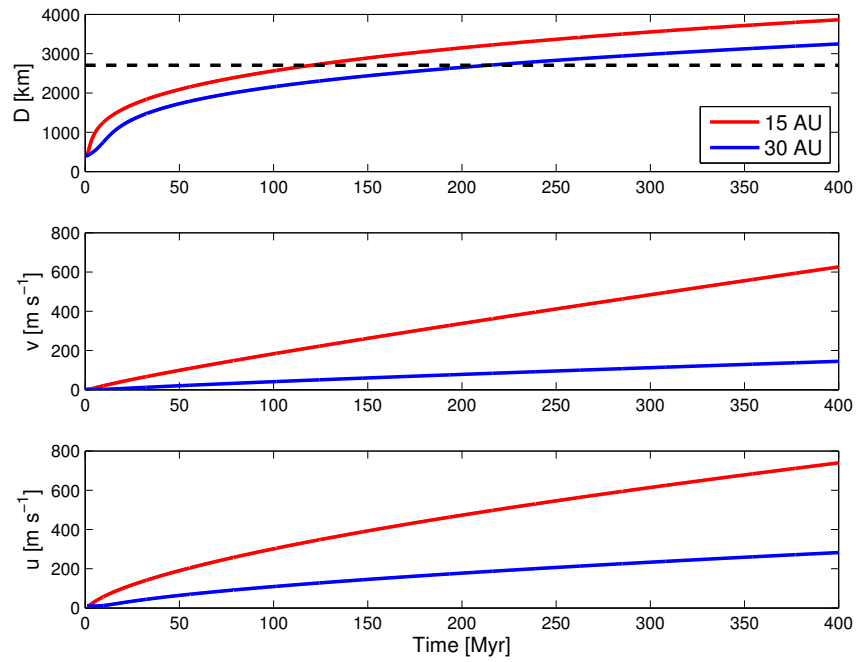

Fig. 12. Evolution of the diameter $D$ (Triton dashed) and random velocity $v$ of the largest TNOs in the primordial disk (upper and middle panels) according to the two-groups approximation (Goldreich et al. 2004). Evolution of random velocity $u$ of comets (lower panel).
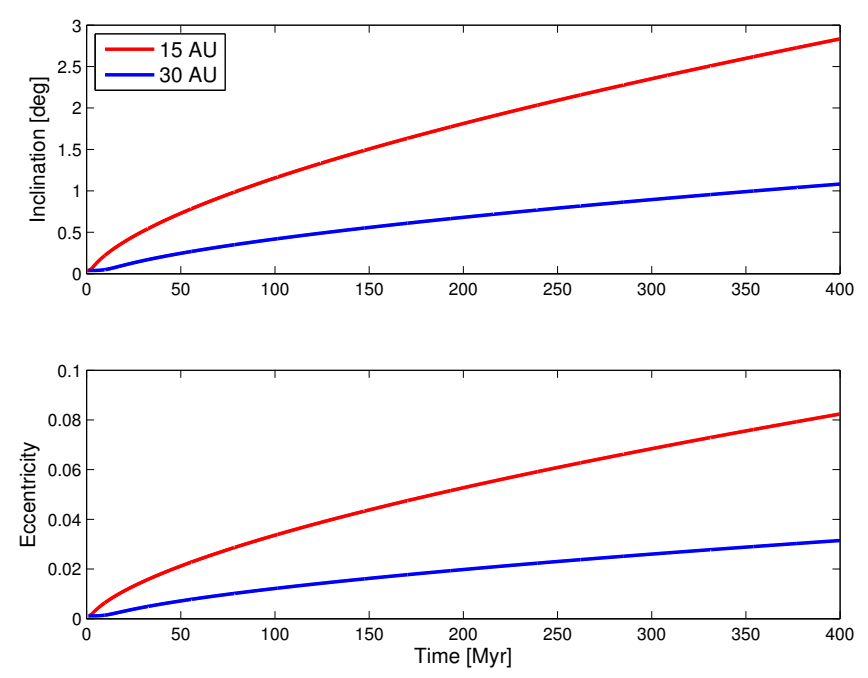

Fig. 13. Evolution of inclination $i$ and eccentricity $e$ for cometesimals and small TNOs as function of time according to Eq. (8).

and to $h=1.13 \mathrm{AU}$ at $r_{\mathrm{h}}=30 \mathrm{AU}$. Thus the volume $V$ containing small bodies grows by a factor of 110-150. On average the small bodies are evenly distributed within a disk of thickness $d=45 \tan i$ [AU] stretching from 15 AU to 30 AU. Meanwhile, velocities grow by a factor of $28-74$, thus viscous stirring tends to reduce collision frequencies $(\propto u / V)$ with time.

\subsubsection{Evolution of the cometesimal size distribution}

The average random velocity in the primordial disk is $\langle u\rangle \approx$ $40 \mathrm{~m} \mathrm{~s}^{-1}$ during the first $\sim 25 \mathrm{Myr}$, in other words, accretion velocities are higher than in the solar nebula. We therefore expect small cometesimals to break upon impact with larger ones, and smear to flat structures similar to the "talps" discussed by Belton et al. (2007). Meter-sized structures may remain intact if their strengths exceed the impact pressure. From Fig. 11 we expect $\psi \approx 45 \%$ and corresponding densities $\rho=$ $990 \mathrm{~kg} \mathrm{~m}^{-3}$ (composition A) or $\rho=670 \mathrm{~kg} \mathrm{~m}^{-3}$ (composition B) on average. Therefore, rather compact concentric layers form around more porous cores that remain uncompacted, suggesting

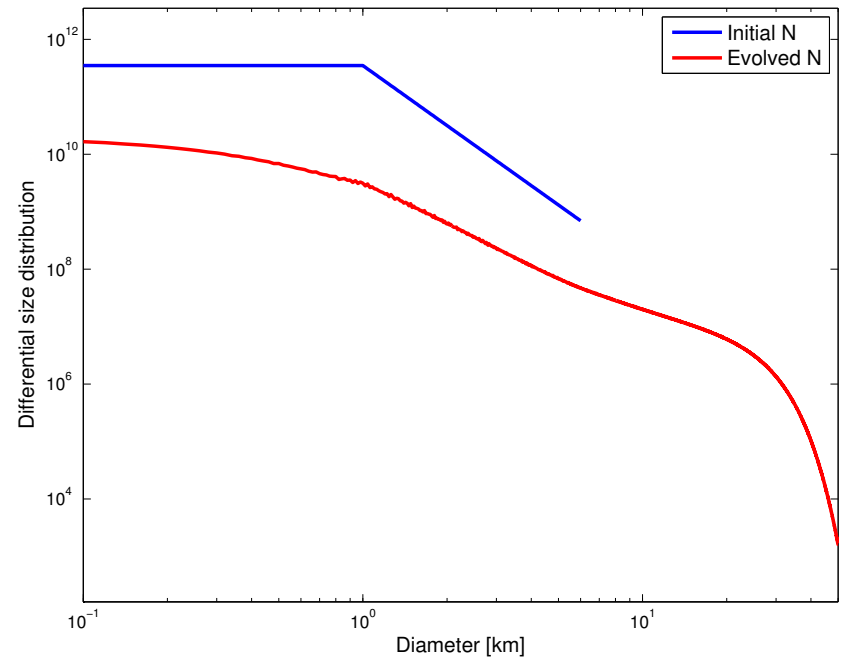

Fig. 14. The initial size distribution of cometesimals (blue) resulting from $\sim 3 \mathrm{Myr}$ of hierarchical agglomeration in the solar nebula combined with selective accretion by TNOs. It is a $q=3.47$ power law at $D \geq 1 \mathrm{~km}$ but flattens at $0.1 \leq D \leq 1 \mathrm{~km}$. The size distribution evolves through low-velocity ( $u=40 \mathrm{~m} \mathrm{~s}^{-1}$ ) mergers during the first $25 \mathrm{Myr}$ of the gas-free primordial disk (red) according to Eq. (9).

$\rho_{\text {bulk }} \sim 500 \mathrm{~kg} \mathrm{~m}^{-3}$. Porosity variation among layers is expected because low- and high-velocity assembly epochs may have alternated during the considered $25 \mathrm{Myr}$. Cometesimals impacting at unusually low speed may have survived intact.

The time evolution of the size distribution has been studied by using a numerical code that divides the $D=0.1-50 \mathrm{~km}$ interval into $20 \mathrm{~m}$-wide bins and that considers all permutations of projectiles and targets of various sizes and calculates their collision frequencies during short time intervals. Net removal or addition of objects in each size bin due to mergers is tracked as function of time while ensuring mass conservation. The volume containing the bodies (height $h=0.01 \mathrm{AU}$ times surface area $A_{\mathrm{d}}$ ) and the relative velocity $u=40 \mathrm{~m} \mathrm{~s}^{-1}$ are assumed to be fixed. In effect we are solving a system of coupled differential equations of the type

$\frac{\mathrm{d} N_{\mathrm{j}}}{\mathrm{d} t}=\frac{\pi u}{4 A_{\mathrm{d}} h}\left(\sum_{i=0}^{j-1} \sum_{l=i}^{j-1} B_{i l} N_{i}\left(D_{i}+D_{l}\right)^{2}-\sum_{i} N_{i}\left(D_{i}+D_{j}\right)^{2}\right)$

where $B_{i l}=1$ if the mass sum of bodies $i$ and $l$ are such that the merged body ends up in the bin of $j$, otherwise $B_{i l}=0$.

Figure 14 shows the initial size distribution as a blue curve (Sect. 4.3). It carries a total mass of $1.49 M_{\oplus}$ distributed among $1.59 \times 10^{13}$ bodies. The largest bodies have $D=6 \mathrm{~km}$. After $25 \mathrm{Myr}$ of evolution the cometesimals have been heavily depleted (red curve in Fig. 14). There are $4.51 \times 10^{11}$ small comets $(0.1 \leq D \leq 10 \mathrm{~km})$ carrying a mass of $0.12 M_{\oplus}$. About $49 \%$ of these have $D=100-500 \mathrm{~m}$ while $2.28 \times 10^{11}$ have $0.5 \leq$ $D \leq 10 \mathrm{~km}$. Most of the mass $\left(1.37 M_{\oplus}\right)$ has been placed in $7.59 \times 10^{9}$ newly formed large comets $(10 \mathrm{~km} \leq D \leq 50 \mathrm{~km})$. The size distribution has become more shallow with $q=2.39$ in the $1 \mathrm{~km} \leq D \leq 6 \mathrm{~km}$ region. This corresponds to a cumulative power law index of $\alpha_{\mathrm{c}}=q-1=1.39$.

The masses and number of nuclei in various size intervals are now $1.6 \times 10^{-4} M_{\oplus}\left(2.22 \times 10^{11}\right.$ nuclei $)$ at $0.1 \leq D \leq 0.5 \mathrm{~km}$, $0.0067 M_{\oplus}\left(1.88 \times 10^{11}\right.$ nuclei $)$ at $0.5 \leq D \leq 2 \mathrm{~km}, 0.016 M_{\oplus}$ $\left(2.76 \times 10^{10}\right.$ nuclei $)$ at $2 \leq D \leq 4 \mathrm{~km}, 0.023 M_{\oplus}\left(7.32 \times 10^{9} \mathrm{nu}-\right.$ clei) at $4 \leq D \leq 6 \mathrm{~km}, 0.077 M_{\oplus}\left(5.98 \times 10^{9}\right.$ nuclei $)$ at $6 \leq D \leq 10 \mathrm{~km}$. 

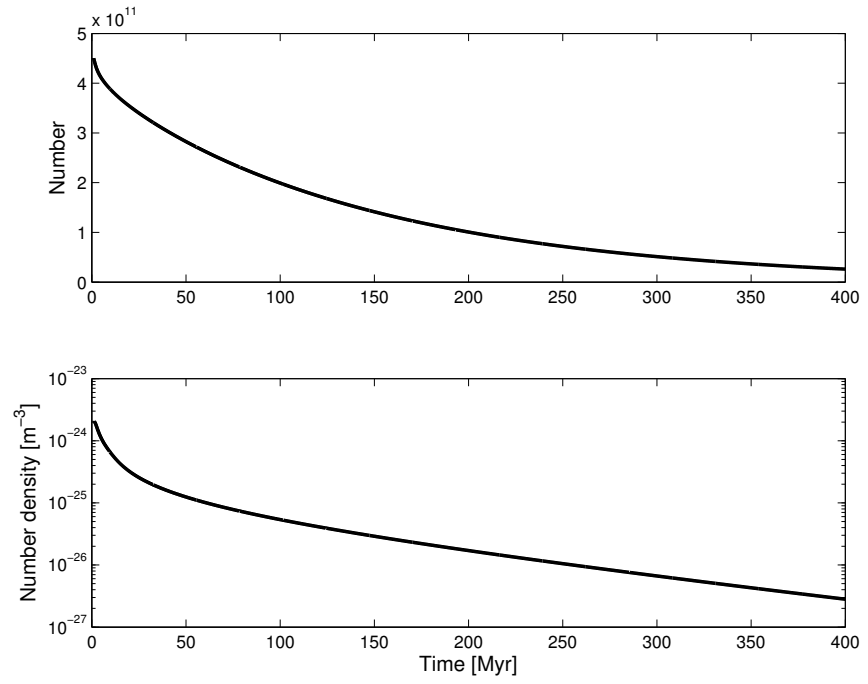

Fig. 15. Reduction in the number (upper panel) and number density (lower panel) of $D \leq 10 \mathrm{~km}$ comet nuclei due to consumption by TNOs according to Eq. (10).

An important aspect of the formation of these nuclei is that the relative velocities no longer are size dependent. In the solar nebula the gas drag prevented collisions among equal-sized cometesimals. The mild viscous stirring in the early gas-free primordial disk removes this limitation. Thus, the last step in comet formation could very well be the merger of two cometesimals of similar size. A large cometesimal with compacted outer layers may behave differently during a collision than a small unlayered one. The latter may break upon impact into a "talps" but the hardened surface of a layered cometesimal may protect it during collisions, allowing it to maintain its shape. We thus suggest that numerous bi-lobed nuclei formed during the first few ten Myr after solar nebula gas dispersal, and that the lobes are individually layered, due to viscous stirring by TNOs.

We now investigate the fate of the comet nuclei during the rest of the $0.4 \mathrm{Gyr}$ interval. The number density of comets evolves for two reasons; (1) the volume containing nuclei increases due to viscous stirring; (2) comets are consumed by TNOs. The collision frequencies among comets evolve for two reasons; (1) the change in number density; (2) the gradual growth of $u$ due to viscous stirring. The sweep-up of comets by TNOs is modeled as

$$
\frac{\mathrm{d} N_{\mathrm{com}}}{\mathrm{d} t}=\frac{\pi u(t) N_{\mathrm{com}}(t)}{A_{\mathrm{d}} h(t)} \sum_{i} \mathcal{N}_{i} D_{i}^{2}\left(1+\left(v_{\mathrm{esc}} / u\right)^{2}\right)
$$

where $\mathcal{N}_{i}$ is the size distribution of TNOs divided into fine bins centered on diameters $D_{i}$ (assuming comets have negligible sizes compared to TNOs). Gravitational focusing is taken into account. The evolution of $N_{\text {com }}$ is seen in Fig. 15. Accretion of $D \leq 10 \mathrm{~km}$ comet nuclei onto TNOs reduces their number by a factor of 17 during $0.4 \mathrm{Gyr}$.

In the first $25 \mathrm{Myr}$ collision velocities among comets are so low that they grow through mergers and remain porous. However, when $u$ increases above $100 \mathrm{~m} \mathrm{~s}^{-1}$ comet nuclei risk local compaction or global pulverization. We estimate a transition diameter $D_{\text {tran }}$ between compactive and destructive projectiles onto a $67 \mathrm{P}$-sized target with $D_{67 \mathrm{P}}=3.3 \mathrm{~km}$ (diameter of volume-equivalent sphere) as follows. The specific energy of icy bodies is $Q_{\mathrm{D}}^{*}=7000 \mathrm{~J} \mathrm{~kg}^{-1}$ at $u=500 \mathrm{~m} \mathrm{~s}^{-1}$ (Benz \& Asphaug 1999). A projectile of mass $m_{\mathrm{p}}$ is destructive when its kinetic energy $E_{\mathrm{p}}=m_{\mathrm{p}} u^{2} / 2$ matches the energy needed to disrupt a body
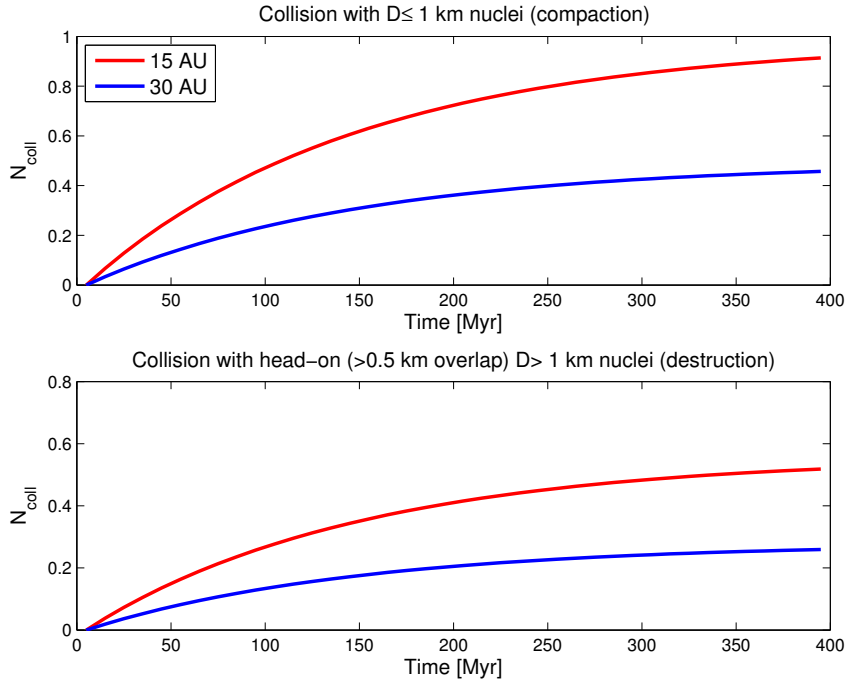

Fig. 16. Number of collisions between a target with $D_{67 \mathrm{P}}=3.3 \mathrm{~km}$ and compacting projectiles with $D \leq D_{\text {tran }}=1 \mathrm{~km}$ (upper panel) according to Eq. (11), or destructive projectiles with $D_{\text {tran }} \leq D \leq 10 \mathrm{~km}$ (lower panel) according to Eq. (12).

like $67 \mathrm{P}$ and disperse half of its mass, $E_{\text {tran }}=\pi \rho_{\text {bulk }} Q_{\mathrm{D}}^{*} D_{67 \mathrm{P}}^{3} / 6$. For $280 \leq u \leq 740 \mathrm{~m} \mathrm{~s}^{-1}$ (Sect. 4.4) we obtain $0.97 \leq D_{\text {tran }} \leq$ $1.85 \mathrm{~km}$. Because high velocities are reached only toward the end of the primordial disk lifetime we apply $D_{\text {tran }}=1 \mathrm{~km}$.

To calculate the number of compactive impacts on a 67Psized nucleus we numerically solve the differential equation

$\frac{\mathrm{d} N_{\text {comp }}}{\mathrm{d} t}=\frac{1}{4} \pi A_{\mathrm{d}}(t) h(t) \sum_{i} N_{i}(t)\left(D_{i}+D_{67 \mathrm{P}}\right)^{2}$

where the summation is made over bins $i$ (containing $N_{i}(t)$ nuclei) for which the projectile diameters fulfill $D_{i} \leq D_{\text {tran }}$. Timedependent loss of projectiles by accretion onto comets and TNOs is considered. The result is seen in the upper panel of Fig. 16. The number of impacts statistically approaches $N_{\text {comp }}=0.91$ at $15 \mathrm{AU}$ and $N_{\text {comp }}=0.47$ at $30 \mathrm{AU}$ after $400 \mathrm{Myr}$. Thus, if the probability of avoiding a compactive collision is taken as $P_{\text {comp }}=\exp \left(-N_{\text {comp }}\right)$ (Morbidelli \& Rickman 2015) the fraction of 67P-sized nuclei avoiding such impacts is $\sim 40 \%$ at $15 \mathrm{AU}$ and $\sim 63 \%$ at $30 \mathrm{AU}$. About $37 \%-60 \%$ of the $67 \mathrm{P}-$ like population statistically experience a single hit by a projectile. Of these, $67 \%$ have $D \leq 500 \mathrm{~m}$.

We calculate the number of destructive impacts $N_{\text {des }}$ from

$\frac{\mathrm{d} N_{\mathrm{des}}}{\mathrm{d} t}=\frac{1}{4} \pi A_{\mathrm{d}}(t) h(t) \sum_{i} N_{i}(t)\left(D_{i}+D_{67 \mathrm{P}}-2 d_{\text {graz }}\right)^{2}$

now summing over bins $i$ for which $D_{\text {tran }}<D_{i} \leq 10 \mathrm{~km}$. Impacts with $D>10 \mathrm{~km}$ objects already is accounted for in Eq. (10). According to Eq. (12), $N_{\text {des }}=0.74$ at $15 \mathrm{AU}$ and $N_{\text {des }}=0.37$ at $30 \mathrm{AU}$, thus $48 \%-69 \%$ of the $67 \mathrm{P}$-sized nuclei do not experience destructive impacts in $400 \mathrm{Myr}$. However, some projectiles cause grazing impacts that only affect an outer rim on the target of thickness $d_{\text {graz }}$. If $d_{\text {graz }}=500 \mathrm{~m}$ in Eq. (12) the lower panel in Fig. 16 shows that $N_{\text {des }}=0.52$ at $15 \mathrm{AU}$ and $N_{\text {des }}=0.26$ at $30 \mathrm{AU}$. This means that $59 \%-77 \%$ of the $67 \mathrm{P}$-sized nuclei survive, though some may experience local surface damage due to a "near-miss".

Equation (12) was compared with a more realistic calculation of the intrinsic collision probability $P_{\text {int }}$ using the algorithm of Wetherill (1967) in a computer code provided to us 
Table 1. Mass fractions ( $\%$ of $M_{\mathrm{t}}$ ) of escaping debris during TNO collisions at velocities relevant for the primordial disk (Fig. 12) according to Eqs. (13)-(17).

Collisions among TNOs

\begin{tabular}{rrrrrrr}
\hline \hline Projectile $D[\mathrm{~km}]$ & Target $D[\mathrm{~km}]$ & $v_{\text {rel }}=30 \mathrm{~m} \mathrm{~s}^{-1}$ & $100 \mathrm{~m} \mathrm{~s}^{-1}$ & $200 \mathrm{~m} \mathrm{~s}^{-1}$ & $500 \mathrm{~m} \mathrm{~s}^{-1}$ & $700 \mathrm{~m} \mathrm{~s}^{-1}$ \\
\hline 50 & 50 & 0.13 & 2.02 & 9.65 & 75.86 & 161.7 \\
100 & 100 & 0.05 & 0.80 & 3.82 & 30.00 & 63.97 \\
200 & 200 & 0.02 & 0.32 & 1.51 & 11.86 & 25.29 \\
50 & 100 & 0.01 & 0.15 & 0.70 & 5.52 & 11.78 \\
50 & 200 & - & 0.01 & 0.03 & 0.24 & 0.50 \\
100 & 200 & - & 0.06 & 0.28 & 2.18 & 4.66 \\
100 & 300 & - & 0.01 & 0.04 & 0.35 & 0.75 \\
\hline
\end{tabular}

by P. Farinella and D. Davis. A simulation with $0 \leq e \leq 0.03$ and $0 \leq i \leq 2^{\circ}$ at $15 \leq r_{\mathrm{h}} \leq 18 \mathrm{AU}$ yielded $P_{\text {int }}=2.7 \times$ $10^{-20} \mathrm{~km}^{-2} \mathrm{yr}^{-1}$ and $\langle u\rangle=0.19 \mathrm{~km} \mathrm{~s}^{-1}$. This corresponds to conditions at $15 \mathrm{AU}$ and $t \lesssim 110 \mathrm{Myr}$ in Figs. 12-13, for which Eq. (12) suggests $P_{\text {int }}=6.3 \times 10^{-20} \mathrm{~km}^{-2} \mathrm{yr}^{-1}$. Another simulation with $0 \leq e \leq 0.01$ and $0 \leq i \leq 0.5^{\circ}$ at $30 \leq r_{\mathrm{h}} \leq 33 \mathrm{AU}$ yielded $P_{\text {int }}=3.4 \times 10^{-21} \mathrm{~km}^{-2} \mathrm{yr}^{-1}$ and $\langle u\rangle=0.2 \mathrm{~km} \mathrm{~s}^{-1}$. This corresponds to conditions at $30 \mathrm{AU}$ and $t \lesssim 200 \mathrm{Myr}$ in Figs. 1213, for which Eq. (12) implies $P_{\text {int }}=2.5 \times 10^{-20} \mathrm{~km}^{-2} \mathrm{yr}^{-1}$. This suggests that Eq. (12) exaggerates $P_{\text {int }}$ by a factor of 2-7 and that the vast majority of 67P-sized nuclei should escape both compaction and destruction. Gomes et al. (2005) associated the LHB with ice giant migration but if these events are unrelated then comets may have spent $\ll 400 \mathrm{Myr}$ in the primordial disk and collision risks are reduced accordingly. We do not think a $\gg 400$ Myr primordial disk lifetime is realistic because a collisional cascade would be unavoidable and we do not find support for comets being collisional rubble piles.

\subsubsection{Debris production by TNOs}

We here apply a collision and fragmentation model (see Benavidez \& Campo Bagatin 2009, and references therein) to explore the effect of collisions among TNO-sized bodies in the primordial disk. We focus on the production of collisional fragments that reach escape velocity. The relative kinetic energy in a collision is

$E_{\text {rel }}=\frac{1}{2} \frac{M_{\mathrm{p}} M_{\mathrm{t}}}{M_{\mathrm{p}}+M_{\mathrm{t}}} v_{\text {rel }}^{2}$

where $M_{\mathrm{p}}$ and $M_{\mathrm{t}}$ are the masses of projectiles and targets. $E_{\mathrm{rel}} / 2$ is delivered to the target of which a fraction $f_{\mathrm{KE}}$ is kinetic energy shared among its fragments,

$f_{\mathrm{KE}}=\frac{k}{k-2} 2^{-2 / k} \frac{v_{\mathrm{esc}}^{2}}{Q_{\mathrm{D}}^{*}}$,

where $k$ is defined in Eq. (17). We apply the same $Q_{\mathrm{D}}^{*}$-value as in Sect. 4.4.1. The kinetic energy of the fragments is

$E_{\mathrm{fr}}=\frac{1}{2} f_{\mathrm{KE}} E_{\mathrm{rel}}$

and the minimum speed of ejecta is

$V_{\min }=\sqrt{\frac{k-2}{k} \frac{E_{\mathrm{fr}}}{M_{\mathrm{t}}}}$.

This analytical model estimates the mass of the ejecta that acquires a velocity $>v_{\text {esc }}$ expressed as a fraction $f_{\text {esc }}$ of the target mass,

$f_{\text {esc }}=\left(\frac{v_{\text {esc }}}{V_{\text {min }}}\right)^{-k}$
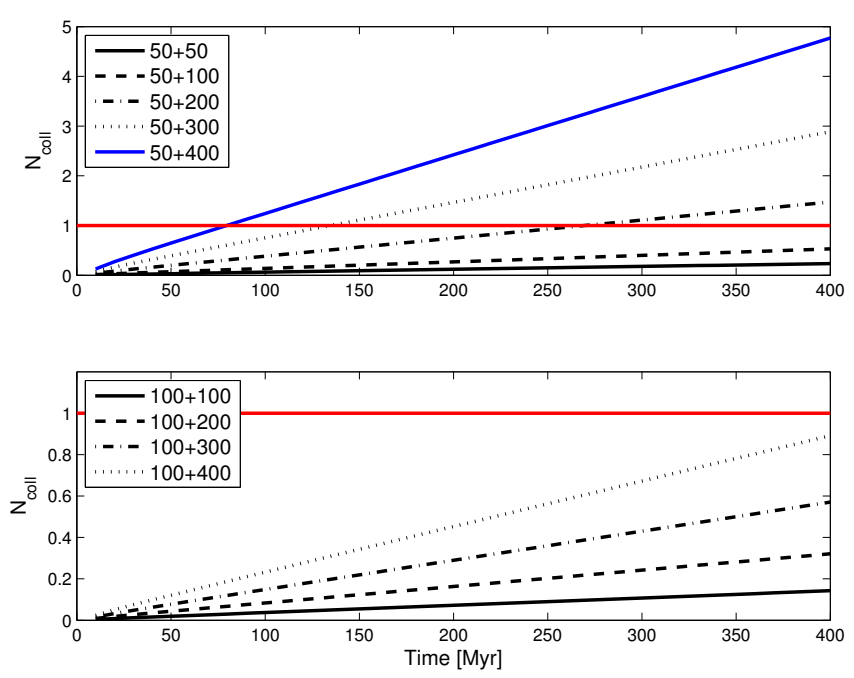

Fig. 17. Number of collisions experienced by various TNO pairs as function of time according to Eq. (18). Upper panel: $50 \mathrm{~km}$ projectiles. Lower panel: $100 \mathrm{~km}$ projectiles.

with $k=\frac{9}{4}$ (Benavidez \& Campo Bagatin 2009).

According to Table $1, v_{\text {rel }} \leq 100 \mathrm{~m} \mathrm{~s}^{-1}$ collisions among equal-sized bodies with $D=50-200 \mathrm{~km}$ produce at most a few percent escaping debris. However, at $v_{\text {rel }}=200 \mathrm{~m} \mathrm{~s}^{-1}$, collisions among $D=50 \mathrm{~km}$ bodies produce significant debris and if $v_{\text {rel }}=500-700 \mathrm{~m} \mathrm{~s}^{-1}$ this is true also for $D=100-200 \mathrm{~km}$ bodies. We calculate the number of such collisions by evaluating

$N_{\text {coll }}(t)=N_{\left[R_{\mathrm{t}}, R_{\mathrm{t}}+10\right]} \frac{\langle u\rangle \pi t}{\left\langle A_{\mathrm{d}} h\right\rangle}\left(R_{\mathrm{p}}+R_{\mathrm{t}}\right)^{2}$

where $N_{\left[R_{\mathrm{t}}, R_{\mathrm{t}}+10\right]}$ is the number of TNOs with diameters on the interval $[D, D+10 \mathrm{~km}], A_{\mathrm{d}}$ is the surface area of the disk, and we average $u(t)$ and $h(t)$ on the interval $[0, t]$.

The results are shown in Fig. 17. It is evident that collisions between equal-sized bodies rarely take place. However, collisions with $M_{\mathrm{p}} \ll M_{\mathrm{t}}$ are common (otherwise the TNOs would not grow in the primordial disk). For example, $D=50 \mathrm{~km}$ bodies are swept up by $D \geq 100 \mathrm{~km}$ bodies. In Table 1 we see that such collisions may produce $6 \%-12 \%$ of escaping debris at $t \approx 400 \mathrm{Myr}$ when $v_{\text {rel }}=500-700 \mathrm{~m} \mathrm{~s}^{-1}$. However if the projectile and/or target are larger the debris production is just a few times $0.01-0.1 \%$. Therefore, the production of small collisional rubble piles in the primordial disk is inefficient.

\subsection{Transfer of TNOs and comets to the EKB, SD, and OC}

Mergers among TNOs reduce their number by merely a factor of 1.53 during $400 \mathrm{Myr}$. The final number of $D \geq 50 \mathrm{~km}$ 
TNOs is therefore $5.6 \times 10^{7}$ in the primordial disk. Combined with $P_{\text {EKB }}=0.19 \%$ (Sect. 4.1) and a $\sim 12 \%$ loss during the last 4 Gyr our model predicts $9.3 \times 10^{4}$ TNOs in the current EKB. Based on the Deep Ecliptic Survey Adams et al. (2014) estimate $(8.07 \pm 2.45) \times 10^{4}$ classical and resonant objects in the EKB with absolute magnitudes $H<10 \mathrm{mag}\left(D>50 \mathrm{~km}\right.$ if $\left.p_{\mathrm{V}}=0.07\right)$. Our model is consistent with these numbers.

The current SD constitutes a fraction $P_{\mathrm{SD}}=0.95 \%$ of the primordial disk (Brasser \& Morbidelli 2013). Our model thus yields $5.3 \times 10^{5}$ objects with $D \geq 50 \mathrm{~km}$ in the current SD. Adams et al. (2014) estimated $(6.6 \pm 1.9) \times 10^{4}$ bodies at $30 \leq a \leq 80 \mathrm{AU}$ that may constitute $\sim 40 \%$ of the total SD (Volk \& Malhotra 2008). If so, there is a total of $(1.7 \pm 0.5) \times 10^{5}$ objects. Our model agrees with this number to within a factor of $2-3$.

Our model has $\sim 350$ unusually large bodies in the primordial disk. During its dispersal one (Triton) was captured by Neptune. The $P_{\text {EKB }}=0.19 \%$ transferred to the EKB corresponds to 0.7 bodies and is statistically consistent with a single Pluto. The $P_{\mathrm{SD}}=0.95 \%$ transferred to the SD corresponds to 3 bodies: Eris and potentially a couple of yet unknown dwarf planets.

According to the current model the final number of comet nuclei in the primordial disk are $1.29 \times 10^{10}$ nuclei at $0.1 \leq D \leq$ $0.5 \mathrm{~km}, 1.09 \times 10^{10}$ nuclei at $0.5 \leq D \leq 2 \mathrm{~km}, 1.60 \times 10^{9}$ nuclei at $2 \leq D \leq 4 \mathrm{~km}, 4.25 \times 10^{8}$ nuclei at $4 \leq D \leq 6 \mathrm{~km}$, and $3.47 \times 10^{8}$ nuclei) at $6 \leq D \leq 10 \mathrm{~km}$. The total number of small comets is $2.62 \times 10^{10}$. We also note that the number of $D=1-$ $10 \mathrm{~km}$ nuclei is $6.62 \times 10^{9}$ and that the number of $D=2-20 \mathrm{~km}$ nuclei is $2.70 \times 10^{9}$. The number of large comets $(10<D \leq$ $40 \mathrm{~km})$ is $4.40 \times 10^{8}$ and there are $8.13 \times 10^{5}$ newly built small TNOs (constituting $\sim 1 \%$ of that population).

The number of comets in the primordial disk with $H<17.3$ $\left(D>2.3 \mathrm{~km}\right.$ if $\left.p_{\mathrm{V}}=0.04\right)$ was predicted as $5 \times 10^{11}$ by Morbidelli et al. (2009) and as $1.9 \times 10^{11}$ by Brasser \& Morbidelli (2013). Our number of $D=2-20 \mathrm{~km}$ nuclei is two orders of magnitude smaller. We note that there is a problem concerning the origin of the LHB which relates to the number of comets in the primordial disk. As noted by Strom et al. (2005), the size distributions of craters on the Moon, Mercury, and Mars resemble that of small main belt asteroids but differ from that of craters on the outer planet satellites. Thus, only a minor fraction of the lunar craters may have been formed by comets, as discussed by Bottke et al. (2012). These authors estimated that the number of comets with $D>2 \mathrm{~km}$ had to be less than $(0.3-2) \times 10^{10}$ to suppress the cometary bombardment sufficiently. This problem was discussed by Bottke et al. (2012) in terms of destruction of small comets by splitting. However, our model has only $2.7 \times 10^{9}$ nuclei with $D>2 \mathrm{~km}$, which is consistent with their estimate without invoking any physical destruction of small comets.

We now proceed to study the transfer of comets to their current reservoirs. Morbidelli \& Rickman (2015) studied collisions in the primordial disk during its violent dispersal. They found that the risk of experiencing a catastrophic collision was 1-2 orders of magnitude smaller than during the long-term residence in the disk. Because we have found that primordial comet nuclei will survive in the primordial disk we assume they survive the transfer as well.

We predict $5.1 \times 10^{6}$ comets with $2 \leq D \leq 20 \mathrm{~km}$ in the EKB. However, the EKB needs to contain $6.7 \times 10^{9}$ comets if it is the only provider of JFCs (Levison \& Duncan 1997). If our analysis is correct the EKB can therefore not be the main source of JFCs. Indeed, following Duncan \& Levison (1997) it is commonly agreed that the SD is the main source of JFCs. Applying $P_{\mathrm{SD}}=0.95 \%$ we predict $N_{1-10}=6.3 \times 10^{7}$ nuclei with $D=1-10 \mathrm{~km}$ and $N_{2-20}=2.6 \times 10^{7}$ nuclei with $D=2-20 \mathrm{~km}$ in the current SD. Bernstein et al. (2004) performed a Hubble Space Telescope (HST) deep survey of $D \gtrsim 15 \mathrm{~km}$ objects. Based on this survey and an extrapolation to $1 \leq D \leq 10 \mathrm{~km}$ Volk \& Malhotra (2008) estimated $3 \times 10^{5}$ nuclei and an upper limit of $2 \times 10^{8}$ nuclei in the $30 \leq r_{\mathrm{h}} \leq 50 \mathrm{AU}$ region (comprising $\sim 15 \%$ of the total SD population). Our prediction of $0.15 N_{1-10}=9.5 \times 10^{6}$ nuclei is well within their range.

We now estimate the number of "visible JFCs" $N_{\text {vis }}$ defined as having total absolute magnitude $H_{\mathrm{T}}<9$ and perihelion distance $q_{\mathrm{h}}<2.5 \mathrm{AU}$ (Levison \& Duncan 1997). Following Brasser \& Morbidelli (2013) we apply $H-H_{\mathrm{T}}=8 \pm 1$, implying visible JFCs have $D \geq 1.4-3.5 \mathrm{~km}$ if $p_{\mathrm{V}}=0.059$ as for $67 \mathrm{P}$ (Sierks et al. 2015). Our model has $N_{1.4-20}=4.19 \times 10^{7}$ comets with $1.4 \leq D \leq 20 \mathrm{~km}$. We calculate the number of visible JFCs as

$N_{\mathrm{vis}}=N_{1.4-20} R_{\mathrm{esc}} f_{\mathrm{JFC}} t_{\mathrm{dyn}} f_{\mathrm{vis}}$

where $R_{\text {esc }}$ is the fractional escape rate from the $\mathrm{SD}, f_{\mathrm{JFC}}$ is the fraction of escapees entering the JFC population, $t_{\text {dyn }}$ is the dynamical lifetime, and $f_{\text {vis }}$ is the fraction of the time during which a JFC has $q_{\mathrm{h}}<2.5$ AU. We apply $f_{\mathrm{JFC}}=0.3$ (Levison \& Duncan 1997; Fernández et al. 2004), $t_{\mathrm{dyn}}=3.3 \times 10^{5} \mathrm{yr}$, and $f_{\mathrm{vis}}=0.07$ (Levison \& Duncan 1994). The $R_{\mathrm{esc}}$-value is uncertain and has been evaluated as $R_{\mathrm{esc}}=(1-2) \times 10^{-10} \mathrm{yr}^{-1}$ (Volk \& Malhotra 2008), $R_{\mathrm{esc}}=2.7 \times 10^{-10} \mathrm{yr}^{-1}$ (Duncan \& Levison 1997; Levison et al. 2006), $R_{\mathrm{esc}}=5 \times 10^{-10} \mathrm{yr}^{-1}$ (Di Sisto \& Brunini 2007), and $R_{\text {esc }}=9.3 \times 10^{-10} \mathrm{yr}^{-1}$ (Emel'yanenko et al. 2004). For this range of $R_{\text {esc }}$-values we obtain $29 \leq N_{\text {vis }} \leq 270$ visible JFCs.

The number of active JFCs with $H_{\mathrm{T}}<9$ and $q_{\mathrm{h}}<2.5 \mathrm{AU}$ has been estimated to be 108-117 (Levison \& Duncan 1997; Di Sisto et al. 2009; Brasser \& Morbidelli 2013). In addition there are dormant comets (see, e.g., Jewitt 2004). The dormant/active ratio $r_{\mathrm{d} / \mathrm{a}}$ for $D>2 \mathrm{~km}$ and $q_{\mathrm{h}}<1.5 \mathrm{AU}$ may be obtained from two numbers: $10 \pm 2$ active JFCs with $D>2 \mathrm{~km}$ and $q_{\mathrm{h}}<1.3 \mathrm{AU}$ (Di Sisto et al. 2009), and the number of cometary Near Earth Objects (NEOs) with $D>2 \mathrm{~km}$. Whitman et al. (2006) listed 15 NEOs with JFC-like orbits, $q_{\mathrm{h}}<1.5 \mathrm{AU}$, and $D>2 \mathrm{~km}$, implying $r_{\mathrm{d} / \mathrm{a}}=1.5 \pm 0.3$. We thus obtain a total of $280 \pm 10$ visual JFCs, which marginally overlaps our upper range $N_{\text {vis }} \leq 270$.

Indeed, earlier estimates based on the capture rate into the JF have tended to result in larger numbers for their source region. This was first done by Levison \& Duncan (1997) in a paper that considered the EKB rather than the SD as the source for captures. They considered JFCs active until a certain time $t_{\mathrm{f}}$ after their entry into orbits with $q_{\mathrm{h}}<2.5 \mathrm{AU}$ (at that point comets were considered extinct). They estimated $t_{\mathrm{f}}=1.2 \times 10^{4} \mathrm{yr}$ by matching the inclination distribution of the model nuclei (for which $i$ increases with time) with the observed one. This physical lifetime was used to estimate $r_{\mathrm{d} / \mathrm{a}}=3.5$. However, these simulations excluded the effect of non-gravitational forces and Hughes et al. (2007) showed that the median inclination of the JFCs grows slower when these forces are included. Thus, Levison \& Duncan (1997) may have underestimated $t_{\mathrm{f}}$ and overestimated $r_{\mathrm{d} / \mathrm{a}}$. Following Fernández et al. (1992), Levison \& Duncan (1997) assumed 40 active JFCs with $H_{\mathrm{T}}<9$ and $q_{\mathrm{h}}<2.0 \mathrm{AU}$. With the steady-state orbital distribution including limited lifetimes this was extrapolated to 108 active JFCs out to $q_{\mathrm{h}}=2.5 \mathrm{AU}$. Because $r_{\mathrm{d} / \mathrm{a}}=3.5$, a total of about $500 \mathrm{JFCs}$ was considered. Using their dynamical lifetime, this number could be translated into a number of similar-sized comets in the source population. In the paper by Duncan \& Levison (1997) the same analysis led to an estimate of $\sim 6 \times 10^{8}$ objects with $H<9$ in the SD. Our $N_{1.4-20}$ is a factor of 14 below that number. This discrepancy is reduced to a factor of $\sim 8$ if instead applying $r_{\mathrm{d} / \mathrm{a}}=1.5$ (a total of $280 \mathrm{JFCs}$ ). Other researchers have come to 
similar conclusions as Duncan \& Levison (1997) regarding the SD comet population size, for example, $(0.6-1.1) \times 10^{9}$ according to Volk \& Malhotra (2008) and (0.4-4.9) $\times 10^{9}$ according to a model by Rickman et al. (2016) that considered size changes due to sublimation.

Volk \& Malhotra (2008) discussed why the number of JFCs seem to require a few times $10^{8}$ comets in the $\mathrm{SD}$, while the HST observations by Bernstein et al. (2004) seem to indicate a number that may be as low as a few times $10^{6}$. Because we predict a few times $10^{7}$ comets in the SD that discussion is highly relevant also in the current context. Among several possibilities Volk \& Malhotra (2008) emphasize that some comets experience tidal splitting during their time as Centaurs. They quote the work by Tiscareno \& Malhotra (2003) who performed orbital integrations for 53 real Centaurs, showing that they collectively passed $8 \times 10^{3}$ times within the Hill radius of a giant planet during their dynamical lifetimes. Thus, discrepancies between the observed number of JFCs and our model could possibly be due to a significant production of smaller nuclei through tidal splitting events.

Another question to consider is the population of the Oort Cloud. Currently, the problem is to explain the large ratio between the estimated numbers of OC and SD objects of similar sizes. Observations suggest that the ratio is $44_{-34}^{+54}$, while simulations of the Nice model scenario suggests a ratio of $12 \pm 1$ if the primordial disk is the only source of OC comets (Brasser \& Morbidelli 2013; Brasser \& Wang 2015). New ideas for OC formation may be thought of, like a possible scenario where the Sun stayed in its birth cluster until the time of the LHB, so that the cloud became initially very compact and later puffed up to its current size by giant molecular cloud encounters. The population ratio between the OC and the SD may thus be increased, but this remains speculative. In any case, if the primordial disk had less than $10^{10}$ comets the OC has to be much less rich in comets than currently thought (Fouchard et al. 2014). Alternatively, the primordial disk was not the only supplier of comets to the OC.

\section{The primordial rubble-pile hypothesis}

The model comet nuclei in Sect. 4 can be described as follows.

1. The typical sizes are $D \sim 1 \mathrm{~m}$ at $t=0.3-0.6 \mathrm{Myr}, D=100 \mathrm{~m}$ at $t=1.2-2.4 \mathrm{Myr}$, and $D=1 \mathrm{~km}$ at $t=1.7-3.6 \mathrm{Myr}$. Comets with $D \approx 40 \mathrm{~km}$ form at $t \approx 25 \mathrm{Myr}$. The slow growth guarantees efficient heat loss and low temperatures even if the pebbles had canonical ${ }^{26} \mathrm{Al}$ abundance at $t=0$ (Sect. 3.2), thus the survival of amorphous water ice (if present), retention of supervolatiles, and preservation of minerals. Granular material on the $\mu \mathrm{m}$-scale therefore has elemental, isotopical, chemical, and mineralogical compositions that resemble those of the free monomer grains in the solar nebula.

2. Mild accretion velocities of $\$ 50 \mathrm{~m} \mathrm{~s}^{-1}$ and absence of aqueous alteration have kept destructive forces to a minimum, thus the physical properties of $\mu \mathrm{m}$-sized grains in comets (e.g., monomer grain size distribution, grain shapes, etc.) resemble those in the solar nebula.

3. The nuclei have acquired specific physical properties during assembly in the solar nebula and young primordial disk. These include high porosity, low density, weak strength, lumpiness on the meter-scale, and nucleus shapes dominated by one or several lobes. Lobes have more porous interiors and less porous external concentric layering. Intact cometesimals and cavities on the $\$ 100 \mathrm{~m}$ scale dominate the interior and are mixed with near-surface strata.

4. Most nuclei have preserved these properties by not having experienced post-formation collisional processing.

We here define such bodies as primordial rubble piles, because they resemble those originally described by Weissman (1986). The word "primordial" does not necessarily require the survival of interstellar material, and does not exclude presence of heavily processed grains from the inner solar system. It here refers to preservation of the chemical and physical properties of solids in the solar nebula that prevailed at the time of planetesimal formation. The phrase "rubble pile" signifies partial structural survival of the building-blocks.

When a primordial rubble pile enters the planetary region processing begins. Solar heating erodes the body and depletes the ice near the moving surface. Diffusing vapor causes subsurface densification and strengthening during recondensation. Extractable organics also cause sintering in dust mantles (Kömle et al. 1996), a process that may create consolidated terrain on 67P (Thomas et al. 2015b). Nuclei may split or fragment due to rotational spin-up or by tidal forces. Should processed bodies still be called "primordial rubble piles"? Here we take a pragmatic approach. The low conductivity means that nucleus erosion keeps pace with the propagation of the orbital heat wave (Gortsas et al. 2011). Thus pristine volatiles and refractories may be encountered within centimeters to meters of the surface, being accessible to landers with drilling devices. Severe tidal break-ups similar to that of comet Shoemaker-Levy 9 (Asphaug \& Benz 1996) may destroy primordial strata and cometesimals. However, large encounter distances are far more common than closer ones and a gentle separation of lobes by weak tidal forces may preserve their structure and porosity. Thus, we extend the phrase "primordial rubble piles" to include processed bodies if they preserve information that allow us to reconstruct the chemical, mineralogical, and physical properties of the solar nebula and primordial disk.

Are most comets primordial rubble piles? We address this question by comparing primordial rubble piles (as defined in Sect. 4) with the observed properties of 67P (Sect. 2) and other comets. We start with properties determined by 3 Myr of growth in the solar nebula. First, primordial rubble piles retain their supervolatiles and amorphous water ice (if present), and avoid aqueous alteration. This is consistent with the high abundance of water and supervolatiles in 67P and in other comets (Sect. 2.6), the tentative detection of amorphous water ice in some comets (Sect. 3.2.4), the absence of phyllosilicates in 81P (Sect. 3.4), lack of widespread $0.7 \mu \mathrm{m}$ absorption features on $67 \mathrm{P}$ (Sect. 2.5), and $T_{\text {spin }}=25-40 \mathrm{~K}$ spin temperatures for ammonia, water, and methane in many comets (Sect. 3.4). Second, the slow growth of primordial rubble piles allows them to incorporate $\$ 3 \mathrm{Myr}$ old grains from the inner solar system throughout their interiors. This is consistent with the richness of crystalline silicates in comets (Sect. 3.1), their homogeneous distribution within 73P, and presence of 1.7-3 Myr CAIs and chondrules in 81P (Sect. 3.6). Third, the most resilient structures in primordial rubble piles have typical diameters of a few meters (Sect. 4.3). This is consistent with the presence of goosebumps, clods, and lumps in 67P with typical diameters of $2.5 \pm 1 \mathrm{~m}$, although their primordial nature needs to be verified (Sect. 2.3). Fourth, we find that $D \lesssim 1 \mathrm{~km}$ cometesimals are selectively accreted by TNOs in the solar nebula (Sect. 4.3) and that such nuclei are not replenished by collisions (Sect. 3.7). This is consistent with the apparent dearth of small JFCs and the potential paucity of small 
craters on the jovian satellites (Sect. 3.5). Fifth, the formation scenario in Sect. 4 does not require strong magnetic fields, consistent with 67P being nonmagnetic (Auster et al. 2015).

Next, we consider the first $25 \mathrm{Myr}$ in the gas-free primordial disk when cometesimals acquired concentric layers and merged into bi-lobed primordial rubble piles (Sect. 4.4.1). Layer formation is consistent with the observed strata that envelope the lobes of 67P individually, and the layers on 9P (Sect. 2.3). The circular "accumulation basins" with stratified walls in Imhotep (Auger et al. 2015) are possibly impact scars formed during accretion. Intact cometesimals and macroscopic voids are mixed with compact strata in primordial rubble piles (Sect. 4.4.1). PRFs having sizes (Fig. 1) consistent with theoretical expectations (Sect. 4.3) and pits on 67P (Sect. 2.3) are possible examples of this. We predict a core density $\rho=440 \mathrm{~kg} \mathrm{~m}^{-3}$ (Sect. 4.3) and layer density $\rho=990 \mathrm{~kg} \mathrm{~m}^{-3}$ (Sect. 4.4.1) for primordial rubble piles with composition A (or a $\rho=210 \mathrm{~kg} \mathrm{~m}^{-3}$ core and $\rho=670 \mathrm{~kg} \mathrm{~m}^{-3}$ layer with composition $\mathrm{B})$. The density ratio between surface and deep interior is thus 2.2-3.2, corresponding to a $9 \%-11 \%$ change over the upper 150 meters on average. This is consistent with CONSERT measurements (Sect. 2.4). If a $D=2.2 \mathrm{~km}$ sphere represents the small lobe of $67 \mathrm{P}$ the bulk density of $67 \mathrm{P}$ (Sect. 2.1) is reproduced for a layer thickness of $70 \pm 20 \mathrm{~m}$ (composition A) to $350 \pm 50 \mathrm{~m}$ (composition $\mathrm{B}$ ). This is roughly what is being observed on the northern hemisphere of 67P (Sect. 2.3). The corresponding tensile strength of primordial rubble piles is $S_{\mathrm{t}} \lesssim 100 \mathrm{kPa}$ (Sect. 3.5), consistent with that of 67P and other comets (Sects. 2.2 and 3.5).

A model nucleus shaped as 67P with core and layer densities for composition $\mathrm{A}$, with a $70 \mathrm{~m}$ shell that envelopes the lobes individually (thus extends into the neck), has $I_{z}-I_{y} \approx 0.12$ (indistinguishable from the moments of inertia of a uniform body). However, most dense layers may have been lost in the heavily eroded southern hemisphere (Keller et al. 2015), and numerous terraces in the north suggest considerable layer erosion there as well. Furthermore, terrace formation suggests differences in sublimation speed, thus density variability among layers, as expected from theory (Sect. 4.4.1). If layering is restricted to a $30^{\circ}$ wide region around the equatorial plane $(200 \mathrm{~m}$ thick to obtain the correct bulk density) we get $I_{z}-I_{y} \approx 0.25$. An extreme model $\left(\rho=100 \mathrm{~kg} \mathrm{~m}^{-3}\right.$ core and $350 \mathrm{~m}$ thick shells in the equatorial region with $\rho=1820 \mathrm{~kg} \mathrm{~m}^{-3}$ ) yields $I_{z}-I_{y} \approx 0.70$. If $67 \mathrm{P}$ has $I_{z}-I_{y} \approx 0.96$ (Sect. 2.4) the mass heterogeneity predicted for primordial rubble piles may not suffice.

Many primordial rubble piles are expected to be bi-lobed (Sect. 4.4.1). This is exemplified by letting all model cometesimals in the $1.8 \leq D \leq 2.6 \mathrm{~km}$ range represent the small lobe of $67 \mathrm{P}$ (the head) with an assigned a common diameter $D=2.2 \mathrm{~km}$. They amount to $1.02 \times 10^{12}$ after solar nebula dispersal and to $2.14 \times 10^{10}$ after $25 \mathrm{Myr}$. We represent the large lobe (the body) by a $D=3.4 \mathrm{~km}$ object. We calculate their collision probabilities using $u=40 \mathrm{~m} \mathrm{~s}^{-1}$ and $h=0.01 \mathrm{AU}$ in Eq. (9). In the beginning of the $25 \mathrm{Myr}$ interval $2.2 \mathrm{Myr}$ passes before a given "body" collides gently with a "head". Thus numerous bi-lobed nuclei form. However, they are likely to sweep up other cometesimals and grow even larger than 67P. Toward the end of the $25 \mathrm{Myr}$ interval the number densities are reduced and it takes $\sim 107 \mathrm{Myr}$ for a "body" to sweep up a "head". The rapid transition from a high bi-lobe formation rate to a low one suggest a mixed population of single- and bi-lobed small comet nuclei with $D \gtrsim 1 \mathrm{~km}$, as observed (Sect. 2.3). For $D \lesssim 1 \mathrm{~km}$ nuclei we expect singlelobed nuclei to dominate, but the existence of a size dependence for nucleus elongation requires observational confirmation.
Primordial rubble piles have a size distribution with $\alpha_{\mathrm{c}}=$ $q-1=1.39$ (Sect. 4.4.1). This is shallower than $\alpha_{\mathrm{c}}^{\mathrm{JFC}}=2.0 \pm 0.2$ that may describe JFCs prior to sublimation (Sect. 3.5). The difference is perhaps explained by tidal splittings of small Centaurs if "accretion in reverse" pushes the size distribution back toward $\alpha_{\mathrm{c}}=2.47$ (Fig. 14). Primordial rubble piles have peak diameters of $D \lesssim 50 \mathrm{~km}$ (Sect. 4.4.1). This is consistent with the existence of large comets like Halley, Neujmin 1, and Hale-Bopp (Sect. 3.7). Active Centaur 95P/Chiron with $D=142 \pm 10 \mathrm{~km}$ (Groussin et al. 2004) is too large to be a primordial rubble pile. For such objects we propose a formation mechanism distinctively different from that of comets (Sect. 4.2) that implies massive loss of supervolatiles (Sect. 3.2.3). This is consistent with 95P producing at least 38-55 times less CO than Hale-Bopp (Bockelée-Morvan et al. 2001).

Mantle material of small partially differentiated TNOs is richer in water ice and phyllosilicates than comets (Sect. 3.7). Though little debris from TNO collisions is expected (Sect. 4.4.2), some foreign mantle material might be swept up by comet nuclei in the primordial disk. This is consistent with a handful of meter-sized unusually bright and spectrally distinct boulders with weak $0.7 \mu \mathrm{m}$ absorption features on $67 \mathrm{P}$ (Sect. 2.5).

In conclusion, we find an overall agreement between the properties of primordial rubble piles and those of comet nuclei.

\section{Discussion}

The concurrent comet and TNO formation scenario in Sect. 4 is somewhat sketchy and is intended to be illustrative, inspirational, and thought-provoking. Its various aspects have to be scrutinized and modeled in detail to better understand its merits and flaws. It may not be unique but we have confidence in it because it explains more data than any other model thus far proposed. The properties of comets are in better agreement with primordial rubble piles than with collisional rubble piles.

The only justification of the collisional rubble-pile scenario concerns the survival of comets against collisions. We find that TNOs do not initiate a collisional cascade (Sect. 4.4.2) until reaching the EKB (Sect. 3.5) and that destructive collisions among comets are rare (Sect. 4.4.1). However, Morbidelli \& Rickman (2015), Rickman et al. (2015) come to the opposite conclusion (67P has a $<10^{-4}$ probability of survival). It is important to understand the reasons for this difference. We have identified three factors; (1) the mass of the primordial disk; (2) the random velocity $u$; (3) planetesimal eccentricities.

During the 1990s hierarchical agglomeration was the only regularly modeled planetesimal growth mechanism. Such growth is slow at low surface densities, thus massive disks were considered necessary to grow the ice giant cores before the solar nebula dispersed. A massive TNO-forming region was also considered necessary to let Pluto reach its current size before its capture into the 3:2 MMR of migrating Neptune (Malhotra 1993). For example, Kenyon \& Luu (1998) suggested $50 M_{\oplus}$ within a $6 \mathrm{AU}$ annulus at $35 \mathrm{AU}$ to grow Pluto in 5-10 Myr. When the EKB turned out to contain only $\sim 0.01 M_{\oplus}$ a "mass deficit problem" had to be solved. The Nice model (Tsiganis et al. 2005) partially did this by locating TNO formation to a primordial disk with $30-50 M_{\oplus}$ at $r_{\mathrm{h}} \lesssim 30-35 \mathrm{AU}$ of which $\lesssim 1 \%$ was relocated to the EKB. That still meant 10-30 times too much mass and a need for substantial collisional grinding (e.g., Charnoz \& Morbidelli 2007). Following that tradition Morbidelli \& Rickman (2015), Rickman et al. (2015) assume $M_{\mathrm{PD}}=35 M_{\oplus}$. 
However, with the discovery that streaming instabilities (Youdin \& Goodman 2005; Johansen et al. 2012) rapidly can by-pass the exceedingly slow initial phase of hierarchical agglomeration one may question if a massive disk is necessary. In Sect. 4.4 we demonstrate that Pluto-size objects may grow on time even if $M_{\mathrm{PD}}=15 M_{\oplus}$. Such a low-mass disk solves the mass deficit problem while rescuing primordial comets from destruction. Tsiganis et al. (2005) note that $M_{\mathrm{PD}}=35-40 M_{\oplus}$ is an upper limit because higher masses yields a too high orbital separation between Jupiter and Saturn. A disk mass as low as $15 M_{\oplus}$ may still provide a sufficient degree of ice giant orbit circularization. We encourage increased efforts in exploring the lowest possible primordial disk mass that is still consistent with the overall Nice scenario, because that may help us understand why primordial comets survived.

Morbidelli \& Rickman (2015), Rickman et al. (2015) consider $2 \times 10^{11}$ potential projectiles with $1 \leq D \leq 100 \mathrm{~km}$ that may hit 67P. Depending on their choice of size distribution, (0.9$5.9) \times 10^{7}$ of these have $50 \leq D \leq 100 \mathrm{~km}$. Our model has $4.51 \times 10^{11}$ comets with $D \leq 10 \mathrm{~km}$ at $t=25 \mathrm{Myr}$ decreasing to $2.62 \times 10^{10}$ at $t=400 \mathrm{Myr}$. We have $(4.7-7.1) \times 10^{7}$ TNOs with $50 \leq D \leq 100 \mathrm{~km}$. The two sets of numbers are similar. Therefore, the difference in 67P survival probability is not due to the number of potential projectiles. The disk masses $\left(15 M_{\oplus}\right.$ versus $\left.35 M_{\oplus}\right)$ do not play a role in this particular context - the mass difference is carried by $D>100 \mathrm{~km}$ bodies that are too few to affect $67 \mathrm{P}$ directly.

However, the indirect effect of the large bodies on the random velocity $u$ is substantial. Morbidelli \& Rickman (2015), Rickman et al. (2015) use $u=600 \mathrm{~m} \mathrm{~s}^{-1}$ throughout the primordial disk lifetime, that is, much higher than our values (Fig. 12) most of the time. They base this on Levison et al. (2011) who considered viscous stirring by $10^{3}$ Pluto-sized bodies for $400 \mathrm{Myr}$. The reasons for our lower $u$ (thus lower collision frequencies) are; (1) small TNOs grow to Pluto-sized objects over time; (2) this growth is slow due to the low $M_{\mathrm{PD}}$; (3) the number of Plutos is a factor of $\sim 3$ lower than considered by Levison et al. (2011). We do not think growth of Pluto-sized bodies is negligible if they spend $400 \mathrm{Myr}$ in a massive disk. They have $v_{\text {esc }}=1.2 \mathrm{~km} \mathrm{~s}^{-1}$ and feed rapidly on $u=0.6 \mathrm{~km} \mathrm{~s}^{-1}$ planetesimals due to efficient gravitational focusing. One then faces the problem of transferring Pluto-sized objects to the EKB/SD while avoiding the $10^{3}$ super-Plutos. We therefore encourage modeling of comet collision frequencies in disks with gradual TNO growth and viscous stirring.

The last problem concerns the eccentricities. According to Fig. 13, $e \lesssim 0.08$ at $r_{\mathrm{h}}=15 \mathrm{AU}$, while $e \lesssim 0.03$ at $r_{\mathrm{h}}=30 \mathrm{AU}$. Figure 1 in Morbidelli \& Rickman (2015) shows $e \lesssim 0.2$ at $r_{\mathrm{h}}=$ $15 \mathrm{AU}$ and $e \lesssim 0.08$ at $r_{\mathrm{h}}=30 \mathrm{AU}$. High eccentricities dramatically increases the number of objects that may cross the orbit of 67P. In fact, Table 1 in Morbidelli \& Rickman (2015) shows significant collision probabilities between objects with semi-major axes $15 \leq a \leq 20 \mathrm{AU}$ and those with $25 \leq a \leq 30 \mathrm{AU}$. This may be the largest reason for the discrepancy in survival probability. For a comet size distribution of $\alpha_{\mathrm{c}}=1.5$ Morbidelli \& Rickman (2015) find that 67P statistically will experience 58 catastrophic impacts at $15 \leq a \leq 20 \mathrm{AU}$ and 12 at $25 \leq a \leq 30 \mathrm{AU}$. Rickman et al. (2015) state 9-11 catastrophic impacts. A reduction of their $u$ by a factor of $\sim 2$ (to conform with Fig. 12) is not sufficient to avoid catastrophic impacts. It is necessary to avoid crossing orbits, in other words, eccentricities must be reduced.

The primordial nature of comet nuclei, that we find difficult to deny, thus teaches us that the primordial disk may have been less massive than previously thought and it must have been dynamically cold. That may be achieved if Triton-like bodies grew slowly from $\leq 400 \mathrm{~km}$ precursors.

\section{Summary and conclusions}

Thanks to the ESA Rosetta mission, and particularly the OSIRIS cameras, we now know that comet 67P consists of two lobes joined by a neck. It has low density, high porosity, and weak strength; it contains supervolatiles and has experienced little to no aqueous alteration. Before merging at low velocity, the two lobes experienced an extensive surface layering process. When combining these findings with data from other spacecraft missions it is becoming increasingly clear that comets formed gently in extreme cold, did not experience thermal processing, and grew to their current sizes during a considerable time period.

The presence of ${ }^{26} \mathrm{Al}$ in the early inner solar system is undeniable and there is little to suggest that short-lived radionuclides avoided the outer solar system. The degree of compaction in some small TNOs and irregular satellites, and spectrophotometric evidence of aqueous alteration, are difficult to explain without short-lived radionuclides. Our current understanding of the primordial disk suggests that collisions were common but here we face a paradox - if comets are collisional fragments, why do they display none of the properties of their thermally processed parent bodies?

We have proposed a concurrent comet and TNO formation scenario that may resolve this problem. It envisions rapid formation of TNOs due to streaming instabilities, with initial sizes $\$ 400 \mathrm{~km}$. The formation of TNOs consumed most pebbles until their number density became too low for that process to continue. The remaining pebbles, constituting a small percentage of the disk mass, then grew to comet nuclei through hierarchical agglomeration. Small comets $(D \lesssim 10 \mathrm{~km})$ formed in $\sim 3 \mathrm{Myr}$, while large comets grew on timescales $\sim 10$ times longer. The low formation velocities led to a fragile nucleus structure with high porosity and low density. The slow growth allowed comets to accrete aged material from the inner solar system and explains why large comets like Hale-Bopp are rich in supervolatiles and have low spin temperatures in spite of the initially high ${ }^{26} \mathrm{Al}$ abundance of their building-blocks. The TNOs formed much earlier and at significant sizes and could not avoid this thermal processing, which led to significant compaction and aqueous alteration in these bodies. The accretional growth of TNOs, in extreme cases to the sizes of Triton, Pluto, and Eris, was so slow that the viscous stirring they caused remained weak until the end of the primordial disk lifetime. The mild increase in collision velocities caused by this viscous stirring led to the formation of denser external layers on cometesimals, on top of the porous cores formed previously in the solar nebula. Furthermore, viscous stirring allowed similarly-sized objects to meet for the first time, giving many comet nuclei a bi-lobed shape. We propose that the dynamical coldness of the primordial disk suppressed collision probabilities sufficiently to guarantee the survival of comet nuclei until the relocation to their current reservoirs.

In conclusion, it is becoming increasingly clear that comets are indeed ancient time-capsules that preserve an unparalleled wealth of information on early solar system history. Their lack of thermal and collisional processing make them excellent tools for understanding the chemical, mineralogical, and physical properties of the solar nebula. Their internal structure offers access to evidence of the accretion processes that formed these objects. Grown in the shadow of the large trans-Neptunian bodies, and after having survived essentially undamaged for $4.6 \mathrm{Gyr}$, the 
primordial comet $67 \mathrm{P} /$ Churyumov-Gerasimenko is a worthy target of the Rosetta mission.

Acknowledgements. We are grateful to Paul Weissman and an anonymous referee for their comments that substantially improved our paper. We thank Bastian Gundlach and Jürgen Blum for sharing unpublished results from their laboratory measurements. OSIRIS was built by a consortium led by the MaxPlanck-Institut für Sonnensystemforschung, Göttingen, Germany, in collaboration with CISAS, University of Padova, Italy, the Laboratoire d'Astrophysique de Marseille, France, the Instituto de Astrofísica de Andalucía, CSIC, Granada Spain, the Scientific Support Office of the European Space Agency, Noordwijk, The Netherlands, the Instituto Nacional de Técnica Aeroespacial, Madrid, Spain, the Universidad Politéchnica de Madrid, Spain, the Department of Physics and Astronomy of Uppsala University, Sweden, and the Institut für Datentechnik und Kommunikationsnetze der Technischen Universität Braunschweig, Germany. The support of the national funding agencies of Germany (DLR), France (CNES), Italy (ASI), Spain (MEC), Sweden (SNSB), and the ESA Technical Directorate is gratefully acknowledged. We thank the Rosetta Science Groun Segment at ESAC, the Rosetta Mission Operations Centre at ESOC, and the Rosetta Project at ESTEC for their outstanding work enabling the science return of the Rosetta Mission.

\section{References}

Abe, S., Mukai, T., Hirata, N., et al. 2006, Science, 312, 1344

Adams, E. R., Gulbis, A. A. S., Elliot, J. L., et al. 2014, AJ, 148, 55

A'Hearn, M. F., Belton, M. J. S., Delamere, W. A., et al. 2005, Science, 258, 258 A’Hearn, M. F., Belton, M. J. S., Delamere, W. A., et al. 2011, Science, 332 1396

A'Hearn, M. F., Feaga, L. M., Keller, H. U., et al. 2012, ApJ, 758, 29

Alibert, Y., Mousis, O., Mordasini, C., \& Benz, W. 2005, ApJ, 626, L57

Altwegg, K., Balsiger, H., Bar-Nun, A., et al. 2015, Science, 347, 1261952

Amari, S., Zinner, E., \& Gallino, R. 2012, in AIP Conf. Ser. 1484, eds

S. Kubono, T. Hayakawa, T. Kajino, et al., 57

André, P., \& Montmerle, T. 1994, ApJ, 420, 837

Asphaug, E., \& Benz, W. 1996, Icarus, 121, 225

Auger, A.-T., Groussin, O., Jorda, L., et al. 2015, A\&A, 583, A35

Auger, A.-T., Groussin, O., Jorda, L., et al. 2016, Icarus, submitted

Auster, H.-U., Apathy, I., Berghofer, G., et al. 2015, Science, 349, 5102

Baer, J., \& Chesley, S. R. 2008, Celest. Mech. Dyn. Astron., 100, 27

Balsiger, H., Altwegg, K., Bar-Nun, A., et al. 2015, Sci. Adv., 1, e1500377

Bar-Nun, A., Dror, J., Kochavi, E., \& Laufer, D. 1987, Phys. Rev. B, 35, 2427

Belton, M. J. S. 2014, Icarus, 231, 168

Belton, M. J. S., Thomas, P., Veverka, J., et al. 2007, Icarus, 187, 332

Benavidez, P. G., \& Campo Bagatin, A. 2009, Planet. Space Sci., 57, 201

Benecchi, S. D., Noll, K. S., Grundy, W. M., \& Levison, H. F. 2010, Icarus, 207, 978

Benz, W., \& Asphaug, E. 1999, Icarus, 142, 5

Berger, E. L., Zega, T. J., Keller, L. P., \& Lauretta, D. S. 2011, Geochim. Cosmochim. Acta, 75, 3501

Bernstein, G. M., Trilling, D. E., Allen, R. L., et al. 2004, AJ, 128, 1364

Bibring, J.-P., Langevin, Y., Carter, J., et al. 2015, Science, 349, 671

Biele, J., Ulamec, S., Maibaum, M., et al. 2015, Science, 349, 9816

Bieler, A., Altwegg, K., Balsiger, H., et al. 2015, Nature, 526, 678

Bizzarro, M., Baker, J. A., \& Haack, H. 2004, Nature, 431, 275

Blum, J., \& Wurm, G. 2008, ARA\&A, 46, 21

Blum, J., Schräpler, R., Davidsson, B. J. R., \& Trigo-Rodríguez, J. M. 2006, ApJ, 652,1768

Bockelée-Morvan, D., Lellouch, E., Biver, N., et al. 2001, A\&A, 377, 343

Boehnhardt, H. 2004, in Comets II, eds. M. C. Festou, H. U. Keller, \& H. A. Weaver (Tucson: Univ. of Arizona Press), 301

Boss, A. 2007, ApJ, 660, 1707

Boss, A., \& Vanhala, H. A. . T. 2000, Space Sci. Rev., 92, 13

Boss, A. P., Ipatov, S. I., Keiser, S. A., Myhill, E. A., \& Vanhala, H. A. T. 2008, ApJ, 686, L119

Bottke, W. F., Durda, D. D., Nesvorný, D., et al. 2005, Icarus, 175, 111

Bottke, W. F., Nesvorný, D., Vokrouhlický, D., \& Morbidelli, A. 2010, AJ, 139, 994

Bottke, W. F., Vokrouhlický, D., Minton, D., et al. 2012, Nature, 485, 78

Bouchet, L., Jourdain, E., \& Roques, J.-P. 2015, ApJ, 801, 142

Brasser, R., \& Morbidelli, A. 2013, Icarus, 225, 40

Brasser, R., \& Wang, J.-H. 2015, A\&A, 573, A102

Brearley, A. J. 1989, Geochim. Cosmochim. Acta, 53, 2395

Brearley, A. J., Scott, E. R. D., Keil, K., et al. 1989, Geochim. Cosmochim. Acta, 53,2081
Bridges, J. C., Changela, H. G., Nayakshin, S., Starkey, N. A., \& Franchi, I. A. 2002, Earth Planet. Sci. Lett., 341, 186

Brown, M. E. 2013, ApJ, 778, L34

Brownlee, D., Joswiak, D., \& Matrajt, G. 2012, Meteo. Planet. Sci., 47, 453

Brownlee, D. E., Horz, F., Newburn, R. L., et al. 2004, Science, 304, 1764

Capaccioni, F., Coradini, A., Filacchione, G., et al. 2015, Science, 347, 628

Carry, B. 2012, Planet. Space Sci., 73, 98

Castillo-Rogez, J. C., \& Schmidt, B. E. 2010, Geophys. Res. Lett., 37, L10202

Castillo-Rogez, J. C., Matson, D. L., Sotin, C., et al. 2007, Icarus, 190, 179

Castillo-Rogez, J. C., Johnson, T. V., Lee, M. H., Turner, N. J., \& Matson, D. L. 2009, Icarus, 204, 658

Castillo-Rogez, J. C., Johnson, T. V., Thomas, P. C., et al. 2012, Icarus, 219, 86 Charnoz, S., \& Morbidelli, A. 2007, Icarus, 188, 468

Chaussidon, M., Robert, F., \& McKeegan, K. D. 2006, Geochim. Cosmochim. Acta, 70, 224

Choi, Y.-J., Cohen, M., Merk, R., \& Prialnik, D. 2002, Icarus, 160, 300

Ciarletti, V., Levasseur-Regourd, A. C., Lasue, J., et al. 2015, A\&A, 583, A40

Ciesla, F. J. 2009, Icarus, 200, 655

Clark, R. N., Brown, R. H., Jaumann, R., et al. 2005, Nature, 435, 66

Clayton, R. N., Hinton, R. W., \& Davis, A. M. 1988, Phil. Trans. R. Soc. Lond. 325,483

Czechowski, L. 2012, Acta Geoph., 60, 1192

Davidsson, B. J. R. 2001, Icarus, 149, 375

Davidsson, B. J. R., \& Gutiérrez, P. J. 2004, Icarus, 168, 392

Davidsson, B. J. R., \& Gutiérrez, P. J. 2005, Icarus, 176, 453

Davidsson, B. J. R., \& Gutiérrez, P. J. 2006, Icarus, 180, 224

Davidsson, B. J. R., \& Rickman, H. 2014, Icarus, 243, 58

Davidsson, B. J. R., Gutiérrez, P. J., \& Rickman, H. 2007, Icarus, 187, 306

Davidsson, B. J. R., Gutiérrez, P. J., Groussin, O., et al. 2013, Icarus, 224, 154

Davies, J. K., Roush, T. L., Cruikshank, D. P., et al. 1997, Icarus, 127, 238

de Bergh, C., Boehnhardt, H., Barucci, M. A., Lazzarin, M., \& Fornasier, S. 2004, A\&A, 416, 791

De Sanctis, M. C., Capria, M. T., \& Coradini, A. 2001, AJ, 121, 2792

Dell'Oro, A., Camp Bagatin, A., Benavidez, P. G., \& N, R. A. A. 2013, A\&A, 558, A95

Denneau, L., Jedicke, R., Fitzsimmons, A., et al. 2015, Icarus, 245, 1

Descamps, P., Marchis, F., Michalowski, T., et al. 2007, Icarus, 187, 482

Di Sisto, R. P., \& Brunini, A. 2007, Icarus, 190, 224

Di Sisto, R. P., Fernández, J. A., \& Brunini, A. 2009, Icarus, 203, 140

Diehl, R., Dupraz, C., Bennett, K., et al. 1995, A\&A, 298, 445

Diehl, R., Halloin, H., Kretschmer, K., et al. 2006, Nature, 439, 45

Dodson-Robinson, S. E., Bodenheimer, P., Laughlin, G., et al. 2008, ApJ, 688, L99

Dohnanyi, J. S. 1969, J. Geophys. Res., 74, 2531

Duncan, M. J., \& Levison, H. F. 1997, Science, 276, 1670

Duncan, M. J., Levison, H. F. \& Budd, S. M. 1995, AJ, 110, 3073

El-Maarry, M. R., Thomas, N., Garcia Berná, A., et al. 2015a, Geophys. Res. Lett., 42, 5170

El-Maarry, M. R., Thomas, N., Giacomini, L., et al. 2015b, A\&A, 583, A26

Emel'yanenko, V. V., Asher, D. J., \& Bailey, M. E. 2004, MNRAS, 350, 161

Emelyanov, N. V. 2005, A\&A, 438, L33

Ernst, C. M., \& Schultz, P. H. 2007, Icarus, 190, 334

Farinella, P., \& Davis, D. R. 1996, Science, 273, 938

Fernández, J. A., \& Morbidelli, A. 2006, Icarus, 185, 211

Fernández, J. A., Rickman, H., \& Kamél, L. 1992, in Workshop on Periodic Comets, eds. J. A. Fernández, \& H. Rickman (Montevideo: Univ. República), 143

Fernández, J. A., Tancredi, G., Rickman, H., \& Licandro, J. 1999, A\&A, 352, 327

Fernández, J. A., Gallardo, T. \& Brunini, A. 2004, Icarus, 172, 372

Fernández, Y. R., Kelley, M. S., Lamy, P. L., et al. 2013, Icarus, 226, 1138

Flynn, G. J., Wirick, S., Keller, L. P., \& Jacobsen, C. 2009, J. Phys. Conf. Ser., 186, 012085

Fornasier, S., Doressoundiram, A., Tozzi, G. P., et al. 2004, A\&A, 421, 353

Fornasier, S., Hasselmann, P. H., Barucci, M., et al. 2015, A\&A, 583, A30

Foster, N. J., Kearsley, A. T., Burchell, M. J., et al. 2008, ACM Conf. Abstr. 8209

Fouchard, M., Rickman, H., Froeschlé, C., \& Valsecchi, G. B. 2014, Icarus, 231, 110

Frank, D. R., Zolensky, M. E., Le, L., Weisberg, M. K., \& Kimura, M. 2013, Lunar Planet. Sci., 44, 3082

Fraser, W. C., Brown, M. E., Morbidelli, A., Parker, A., \& Batygin, K. 2014, ApJ, 782, 100

Garenne, A., Beck, P., Montes-Hernandez, G., et al. 2014, Geochim. Cosmochim. Acta, 137, 93

Gladman, B. J., Davis, D. R., Neese, C., et al. 2009, Icarus, 202, 104

Goldreich, P., Lithwick, Y., \& Sari, R. 2004, ARA\&A, 42, 549

Gomes, R. S. 2003, Icarus, 161, 404 
Gomes, R. S., Morbidelli, A., \& Levison, H. F. 2004, Icarus, 170, 492

Gomes, R. S., Levison, H. F., Tsiganis, K., \& Morbidelli, A. 2005, Nature, 435 466

Gortsas, N., Kührt, E., Motschmann, U., \& Keller, H. U. 2011, Icarus, 212, 858 Gounelle, M., \& Meibom, A. 2008, ApJ, 680, 781

Gounelle, M., Morbidelli, A., Band, P. A., et al. 2008, in The solar system beyond Neptune, ed. M. A. Barucci, H. Boehnhardt, D. P. Cruikshank, \& A. Morbidelli (Tucson: The University of Arizona Press), 525

Greenberg, J. M. 1998, A\&A, 330, 375

Groussin, O., Lamy, P., \& Jorda, L. 2004, A\&A, 413, 1163

Groussin, O., Sunshine, J. M., Feaga, L. M., et al. 2013, Icarus, 222, 580

Groussin, O., Jorda, L., Auger, A.-T., et al. 2015, A\&A, 583, A32

Grundy, W. M., Noll, K. S., Buie, M. W., et al. 2009, Icarus, 200, 627

Grundy, W. M., Noll, K. S., Nimmo, F., et al. 2013, Icarus, 213, 678

Gulkis, S., Allen, M., von Allmen, P., et al. 2015, Science, 347, 709

Gundlach, B., \& Blum, J. 2015, ApJ, 798, 34

Güttler, C., Krause, M., Geretshauser, R. J., Speith, R., \& Blum, J. 2009, AJ, 701,130

Güttler, C., Blum, J., Zsom, A., Ormel, C. W., \& Dullemond, C. P. 2010, A\&A, 513, A56

Gutiérrez, P. J., Jorda, L., Gaskell, R. W., et al. 2016, A\&A, submitted

Hagenmuller, P., Theile, T. C., \& Scheebeli, M. 2014, Geophys. Res. Lett., 41, 86

Haisch, K. E., Lada, E. A., \& Lada, C. J. 2001, ApJ, 553, L153

Halliday, A. N., \& Kleine, T. 2006, in Meteorites and the early solar system II eds. D. S. Lauretta, \& H. Y. McSween (Tucson: Univ. of Arizona Press), 775

Harker, D. E., Woodward, C. E., Kelley, M. S., et al. 2011, AJ, 141, 26

Harmon, J. K., Nolan, M. C., Giorgini, J. D., \& Howell, E. S. 2010, Icarus, 207, 499

Haruyama, J., Yamamoto, T., Mizutani, H., \& Greenberg, J. M. 1993, J. Geophys Res., 98, 15079

Hässig, M., Altwegg, K., Balsiger, H., et al. 2015, Science, 347, 276

Hayashi, C. 1981, Suppl. Prog. Theo. Phys., 70, 35

Henke, S., Gail, H.-P., Trieloff, M., Schwarz, W. H., \& Kleine, T. 2012, A\&A 537, A45

Hevey, P. J., \& Sanders, I. S. 2006, Meteo. Planet. Sci., 41, 95

Horai, K.-I. 1971, J. Geophys. Res., 76, 1278

Housen, K. R., \& Holsapple, K. A. 2012, Icarus, 219, 297

Housen, K. R., Holsapple, K. A., \& Voss, M. E. 1999, Nature, 402, 155

Hubickyj, O., Bodenheimer, P., \& Lissauer, J. J. 2005, Icarus, 179, 415

Hughes, D. W., Sleep, P. N., Jones, B. W., \& Jones, J. M. 2007, MNRAS, 382 1675

Ishii, H. A., Stadermann, F. J., Floss, C., et al. 2010, LPSC, 41, 2317

Ivezić, Z., Tabachnik, S., Rafikov, R., et al. 2001, AJ, 122, 2749

Jacob, D., Stodolna, J., Leroux, H., Langenhorst, F., \& Houdellier, F. 2009 Meteo. Planet. Sci., 44, 1475

Jacobson, S. A., Marzari, F., Rossi, A., Scheeres, D. J., \& Davis, D. R. 2014, MNRAS Lett., 439, L95

Jarvis, K. S., Vilas, F., Larson, S. M., \& Gaffey, M. J. 2000, Icarus, 145, 445

Jewitt, D. C. 2004, in Comets II, eds. M. C. Festou, U. Keller, \& H. A. Weaver (Tucson: Univ. of Arizona Press), 659

Jewitt, D. C., \& Haghighipour, N. 2007, ARA\&A, 45, 261

Jewitt, D., \& Luu, J. 2001, AJ, 122, 2099

Johansen, A., Oishi, J. S., Low, M.-M. M., et al. 2007, Nature, 448, 1022

Johansen, A., Youdin, A. N., \& Lithwick, Y. 2012, A\&A, 537, A125

Johansen, A., Blum, J., Tanaka, H., et al. 2014, in Protostars and Planets VI, eds

H. Beuther, R. Klessen, C. Dullemond, \& T. Henning (Tucson: University of Arizona Press), 547

Jorda, L., Gaskell, R., Capanna, C., et al. 2016, Icarus, 277, 257

Joswiak, D. J., Brownlee, D. E., Matrajt, G., Westphal, A. J., \& Snead, C. J. 2009, Meteo. Planet. Sci., 44, 1561

Joswiak, D. J., Brownlee, D. E., Matrajt, G., et al. 2012, Meteo. Planet. Sci., 47, 471

Joswiak, D. J., Nakashima, D., Brownlee, D. E., et al. 2014, Geochim. Cosmochim. Acta, 144, 277

Jutzi, M., Michel, P., Benz, W., \& Richardson, D. C. 2010, Icarus, 207, 54

Kawakita, H., Watanabe, J.-I., Ootsubo, T., et al. 2004, ApJ, 601, L191

Kawakita, H., Dello Russo, N., Furusho, R., et al. 2006, ApJ, 643, 1337

Keller, H. U., Kramm, R., \& Thomas, N. 1988, Nature, 331, 227

Keller, H. U., Britt, D., Buratti, B. J., \& Thomas, N. 2004, in Comets II, eds M. C. Festou, U. Keller, \& H. A. Weaver (Tucson: Univ. of Arizona Press), 211

Keller, H. U., Mottola, S., Davidsson, B., et al. 2015, A\&A, 583, A34

Kelley, M. S., \& Wooden, D. H. 2009, Planet. Space Sci., 57, 1133

Kemper, F., Vriend, W. J., \& Tielens, A. G. G. M. 2004, ApJ, 609, 826

Kenyon, S. J., \& Luu, J. X. 1998, ApJ, 115, 2136

Klinger, J. 1980, Science, 209, 271

Klinger, J. 1981, Icarus, 47, 320
Klöck, W., Thomas, K. L., McKay, D. S., \& Palme, H. 1989, Nature, 339, 126 Kofman, W., Herique, A., Barbin, Y., et al. 2015, Science, 249, 639

Kokubo, E., \& Ida, S. 1996, Icarus, 123, 180

Kömle, N. I., Kargl, G., Thiel, K., \& Seiferlin, K. 1996, Planet. Space Sci., 44, 675

Kouchi, A., Kudo, T., Nakano, H., et al. 2002, ApJ, 566, L121

Krot, A. N., Scott, E. R. D., \& Zolensky, M. E. 1995, Meteoritics, 30, 748

Krot, A. N., Yurimoto, H., Hutcheon, I. D., et al. 2007a, Meteo. Planet. Sci., 42, 1197

Krot, A. N., Yurimoto, H., Hutcheon, I. D., et al. 2007b, Geochim. Cosmochim. Acta, 71, 4342

Krot, A. N., Nagashima, K., Bizzarro, M., et al. 2008, ApJ, 672, 713

Kudo, T., Kouchi, A., Arakawa, M., \& Nakano, H. 2002, Meteo. Planet. Sci., 37, 1975

Lamy, P. L., Toth, I., Fernández, Y. R., \& Weaver, H. A. 2004, in Comets II, eds. M. C. Festou, U. Keller, \& H. A. Weaver (Tucson: Univ. of Arizona Press), 223

Lazzarin, M., Barucci, M. A., Boehnhardt, H., et al. 2003, AJ, 125, 1554

Lederer, S. M., \& Vilas, F. 2004, Earth Moon Planets, 92, 193

Lee, C. W., \& Myers, P. C. 1999, ApJS, 123, 233

Lee, T., Papanastassiou, D. A., \& Wasserburg, G. J. 1978, ApJ, 220, L21

Lellouch, E., Santos-Sanz, P., Lacerada, P., et al. 2013, A\&A, 557, A60

Levison, H. F., \& Duncan, M. J. 1994, Icarus, 108, 18

Levison, H. F. \& Duncan, M. J. 1997, Icarus, 127, 13

Levison, H. F., Duncan, M. J., Dones, L., \& Gladman, B. J. 2006, Icarus, 184, 619

Levison, H. F., Morbidelli, A., van Laerhoven, C., Gomes, R., \& Tsiganis, K. 2008, Icarus, 196, 258

Levison, H. F., Morbidelli, A., Tsiganis, K., Nesvorný, D., \& Gomes, R. 2011, AJ, 142,152

Lisse, C. M., VanCleve, J., Adams, A. C., et al. 2006, Science, 313, 635

Lisse, C. M., Kraemer, K. E., Nuth III, J. A., Li, A., \& Joswiak, D. 2007, Icarus, 187,69

Lodders, K. 2003, ApJ, 591, 1220

Lorek, S., Gundlach, B., Lacerda, P., \& Blum, J. 2016, A\&A, 587, A128

Lowry, S. C., Fitzsimmons, A., \& Collander-Brown, S. 2003, A\&A, 397, 329

Luspay-Kuti, A., Hässig, M., Fuselier, S. A., et al. 2015, A\&A, 583, A4

Macke, R. J., Consolmagno, G. J., \& Britt, D. T. 2011, Meteo. Planet. Sci., 46 , 1842

MacPherson, G. J., Davis, A. N., \& Zinner, E. K. 1995, Meteoritics, 30, 365

Malhotra, R. 1993, Nature, 365, 819

Marchi, S., Bottke, W. F., Cohen, B., et al. 2013, Nature Geosci., 6, 303

Marhas, K. K., Goswami, J. N., \& Davis, A. M. 2002, Science, 298, 2182

Marty, B., Palma, R. L., Pepin, R. O., et al. 2008, Science, 319, 75

Masset, F., \& Snellgrove, M. 2001, MNRAS, 320, L55

Massironi, M., Simioni, E., Marzari, F., et al. 2015, Nature, 526, 402

Matson, D. L., Castillo-Rogez, J. C., Schubert, G., Sotin, C., \& McKinnon, W. B. 2009, in Saturn from Cassini-Huygens, eds. M. K. Dougherty, L. W. Esposito, \& S. M. Krimigis (Springer Science), 577

Matzel, J. E. P., Ishii, H. A., Joswiak, D., et al. 2010, Science, 328, 483

McCulloch, M. T., \& Wasserburg, G. J. 1978, ApJ, 220, L15

Meech, K. J., \& Svoreň, J. 2004, in Comets II, eds. M. C. Festou, H. U. Keller, \& H. A. Weaver (Tucson: Univ. of Arizona Press), 317

Meech, K. J., Hainaut, O. R., \& Marsden, B. G. 2004, Icarus, 170, 463

Merényi, E., Földy, L., Szegö, K., Tóth, I., \& Kondor, A. 1990, Icarus, 86, 9

Merk, R., \& Prialnik, D. 2003, Earth Moon Planets, 92, 359

Merk, R., \& Prialnik, D. 2006, Icarus, 183, 283

Miyamoto, M., Fujii, N., \& Takeda, H. 1981, Proc. Lunar Planet. Sci., 12, B1145

Mommert, M., Harris, A. W., Kiss, C., et al. 2012, A\&A, 541, A93

Morbidelli, A., \& Crida, A. 2007, Icarus, 191, 158

Morbidelli, A., \& Rickman, H. 2015, A\&A, 583, A43

Morbidelli, A., Tsiganis, K., Crida, A., Levison, H. F., \& Gomes, R. 2007, AJ, 134, 1790

Morbidelli, A., Levison, H. F., Bottke, W. F., Dones, L., \& Nesvorný, D. 2009, Icarus, 202, 310

Morbidelli, A., Marchi, S., Bottke, W. F., \& Kring, D. 2012, Earth Planet. Sci. Lett., 355, 144

Mottola, S., Lowry, S., Snodgrass, C., et al. 2014, A\&A, 569, L2

Mottola, S., Arnold, G., Grothues, H.-G., et al. 2015, Science, 349, 232

Nakamura, T., Noguchi, T., Tsuchiyama, A., et al. 2008, Science, 321, 664

Nakashima, D., Ushikubo, T., Kita, N. T., et al. 2015, Earth Planet. Sci. Lett., 410, 54

Nesvorný, D. 2015, AJ, 150, 73

Nesvorný, D., Youdin, A. N., \& Richardson, D. C. 2010, AJ, 140, 785

Nesvorný, D., Vokrouhlický, D., \& Morbidelli, A. 2013, AJ, 768, 45

Noguchi, T., Nakamura, T., Okudaira, K., et al. 2007, Meteo. Planet. Sci., 42, 357 
B. J. R. Davidsson et al.: The primordial nucleus of comet $67 \mathrm{P} /$ Churyumov-Gerasimenko

Norris, T. L., Gancarz, A. J., Rokop, D. J., \& Thomas, K. W. 1983, J. Geophys. Res., 88, B331

Notesco, G., \& Bar-Nun, A. 2000, Icarus, 148, 456

Oberst, J., Giese, B., Howington-Kraus, E., et al. 2004, Icarus, 167, 70

O’Brien, D. P., \& Greenberg, R. 2003, Icarus, 164, 334

Ogliore, R. C., Huss, G. R., Nagashima, N., et al. 2012, ApJ, 745, L19

Oklay, N., Vincent, J.-B., Fornasier, S., et al. 2016, A\&A, 586, A80

Ormel, C. W., Spaans, M., \& Tielens, A. G. G. M. 2007, A\&A, 461, 215

Ouellette, N., Desch, S. J., Bizzarro, M., et al. 2009, Geochim. Cosmochim. Acta, 73, 4946

Pan, M., \& Schlichting, H. E. 2012, ApJ, 747, 133

Parker, A. H., \& Kavelaars, J. J. 2010, ApJ, 722, L204

Parker, A. H., \& Kavelaars, J. J. 2012, ApJ, 744, 139

Parker, A. H., Kavelaars, J. J., Petit, J.-M., et al. 2011, ApJ, 743, 1

Pätzold, M., Andert, T., Hahn, M., et al. 2016, Nature, 530, 63

Petit, J.-M., Kavelaars, J. J., Gladman, B. J., et al. 2008, Science, 322, 432

Podolak, M., \& Prialnik, D. 2006, in Comets and the origin and evolution of life, eds. P. J. Thomas, C. Chyba, \& C. McKay (Berlin: Springer-Verlag)

Pollack, J. B., Hubickyj, O., Bodenheimer, P., et al. 1996, Icarus, 124, 62

Pommerol, A., Thomas, N., El-Maarry, M. R., et al. 2015, A\&A, 583, A25

Porco, C. C., West, R. A., McEwen, A., et al. 2003, Science, 299, 1541

Poulet, F., Bibring, J.-P., Mustard, J. F., et al. 2005, Nature, 438, 623

Preusker, F., Scholten, F., Matz, K.-D., et al. 2015, A\&A, 583, A33

Prialnik, D., \& Podolak, M. 1995, Icarus, 117, 420

Prialnik, D., Bar-Nun, A., \& Podolak, M. 1987, ApJ, 319, 993

Prialnik, D., Sarid, G., Rosenberg, E. D., \& Merk, R. 2008, Space Sci. Rev., 138, 147

Qin, L., Dauphas, N., Wadhwa, M., Masarik, J., \& Janney, P. E. 2008, Earth Planet. Sci. Lett., 273, 94

Reach, W. T., Kelley, M. S., \& Vaubaillon, J. 2013, Icarus, 226, 777

Richardson, J. E., Melosh, H. J., Lisse, C. M., \& Carcich, B. 2007, Icarus, 190 357

Rickman, H. 1986, in The Comet Nucleus Sample Return Mission, ed. O. Melita (ESTEC, Noordwijk: ESA Publications Division), 195

Rickman, H., Kamél, L., Festou, M. C., \& Froeschlé, C. 1987, in Symposium on the diversity and similarity of comets, eds. E. J. Rolfe \& B. Battrick (ESTEC, Noordwijk: ESA Publications Division), 471

Rickman, H., Marchi, S., A'Hearn, M. F., et al. 2015, A\&A, 583, A44

Rivkin, A. S., Howell, E. S., Vilas, F., \& Lebofsky, L. A. 2002, in Asteroids III, eds. W. F. Bottke Jr., A. Cellino, P. Paolicchi, \& R. P. Binzel (Tucson: The University of Arizona Press), 235

Robie, R. A., Hemingway, B. S., \& Takei, H. 1982, American Mineralogist, 67, 470

Roskosz, M., \& Leroux, H. 2015, ApJ, 801, L7

Rotundi, A., Sierks, H., Corte, V. D., et al. 2015, Science, 347, 3905

Rubin, A. E. 1997, Meteo. Planet. Sci., 32, 231

Rubin, A. E., Griset, C. D., Choi, B.-G., \& Wasson, J. T. 2009, Meteo. Planet. Sci., 44, 589, 4

Rubin, M., Altwegg, K., Balsiger, H., et al. 2015, Science, 348, 232

Russell, H. W. 1935, J. Am. Ceram. Soc., 18, 1

Ryan, E. L., Mizuno, D. R., Shenoy, S. S., et al. 2015, A\&A, 578, A42

Sagdeev, R. Z., Avanesov, G. A., Ziman, Y. L., et al. 1986, in 20th ESLAB Symposium on the Exploration of Halley's Comet, eds. B. Battrick, E. J. Rolfe, \& R. Reinhard, Vol. II (Noordwijk: ESA Publications Division), 317

Sahijpal, S., \& Goswami, J. N. 1998, ApJ, 509, L137

Santos-Sanz, P., Lellouch, E., Fornasier, S., et al. 2012, A\&A, 541, A92

Schmitt, B., Espinasse, S., Grim, R. J. A., Greenberg, J. M., \& Klinger, J. 1989, ESA SP, 302, 65

Schmitz, S., \& Brenker, F. E. 2008, ApJ, 681, L105

Schmitz, S., Brenker, F. E., Schoonjans, T., et al. 2009, Geochim. Cosmochim. Acta, 73, L5483

Schubert, G., Anderson, J. D., Travis, B. J., \& Palguta, J. 2007, Icarus, 188, 345

Schulz, R., Hilchenbach, M., Langevin, Y., et al. 2015, Nature, 518, 216

Scott, E. R. D., Rubin, A. E., Taylor, G. J., \& Keil, K. 1984, Geochim. Cosmochim. Acta, 48, 1741

Sheppard, S. S., \& Jewitt, D. C. 2003, Nature, 423, 261

Sheppard, S. S., \& Trujillo, C. A. 2010, ApJ, 723, L233

Shi, X., Hu, X., Sierks, H., et al. 2016, A\&A, 586, A7

Shinnaka, Y., Kawakita, H., Kobayashi, H., et al. 2011, ApJ, 729, 81

Shoshany, Y., Prialnik, D., \& Podolak, M. 2002, Icarus, 157, 219

Shu, F., Najita, J., Ostriker, E., et al. 1994, ApJ, 429, 781

Shukolyukov, A., \& Lugmair, G. W. 1993, Science, 259, 1138

Sicilia-Aguilar, A., Hartmann, L., Calvet, N., et al. 2006, ApJ, 638, 897

Sierks, H., Barbieri, C., Lamy, P. L., et al. 2015, Science, 347, 1044

Simon, S. B., Joswiak, D. J., Ishii, H. A., et al. 2008, Meteo. Planet. Sci., 43, 1861

Sitko, M. L., Lisse, C. M., Kelley, M. S., et al. 2011, AJ, 142, 80

Skorov, Y. V., \& Rickman, H. 1999, Planet. Space. Sci., 47, 935
Snodgrass, C., Fitzsimmons, A., Lowry, S. C., \& Weissman, P. 2011, MNRAS, 414,458

Sosa, A., \& Fernández, J. A. 2009, MNRAS, 393, 192

Spencer, J. R., Lebofsky, L. A., \& Sykes, M. V. 1989, Icarus, 78, 337

Spencer, J. R., Stansberry, J. A., Grundy, W. M., \& Noll, K. S. 2006, BA\&AS, 38,546

Spohn, T., Knollenberg, J., Ball, A. J., et al. 2015, Science, 349, 464

Stephens, D. C., \& Noll, K. S. 2006, AJ, 131, 1142

Stern, S. A. 1988, Icarus, 73, 499

Stern, S. A., \& Weissman, P. R. 2001, Nature, 409, 589

Stodolna, J., Jacob, D., \& Leroux, H. 2010, LPSC, 41, 1657

Stodolna, J., Jacob, D., \& Leroux, H. 2012, Geochim. Cosmochim. Acta, 87, 35

Strom, R. G., Malhotra, R., Ito, T., Yoshida, F., \& Kring, D. A. 2005, Science, 309, 1847

Szabó, G. M., Kiss, L. L., Pál, A., et al. 2012, ApJ, 761, 8

Takir, D., \& Emery, J. P. 2012, Icarus, 219, 641

Tanaka, H., Inaba, S., \& Nakazawa, K. 1996, Icarus, 123, 450

Tancredi, G., Fernández, J. A., Rickman, H., \& Licandro, J. 2006, Icarus, 182, 527

Tesfaye Firdu, F., \& Taskinen, P. 2010, Densities of molten and solid alloys of (Fe, $\mathrm{Cu}, \mathrm{Ni}, \mathrm{Co})-\mathrm{S}$ at elevated temperatures - Literature review and analysis, Aalto University Publications in Materials Science and Engineering (Espoo: Aalto University School of Science and Technology)

Thomas, N., Davidsson, B., El-Maarry, M., et al. 2015a, A\&A, 583, A17

Thomas, N., Sierks, H., Barbieri, C., et al. 2015b, Science, 347, 440

Thomas, P. C., Veverka, J., Belton, M. J. S., et al. 2007, Icarus, 187, 4

Thomas, P. C., A'Hearn, M. F., Veverka, J., et al. 2013, Icarus, 222, 550

Thrane, K., Bizzarro, M., \& Baker, J. A. 2006, ApJ, 646, L159

Thrane, K., Nagashima, K., Krot, A. N., \& Bizzarro, M. 2008, ApJ, 680, L141

Tiscareno, M. S., \& Malhotra, R. 2003, AJ, 126, 3122

Tomeoka, K., \& Itoh, D. 2004, Meteo. Planet. Sci., 39, 1359

Trigo-Rodríguez, J. M., \& Llorca, J. 2006, MNRAS, 372, 655

Tsiganis, K., Gomes, R., Morbidelli, A., \& Levison, H. 2005, Nature, 435, 459

Tsuchiyama, A., Mashio, E., Imai, Y., et al. 2009, Annu. Meteo. Soc. Meet., 72, 5189

Vanhala, H. A. T., \& Boss, A. P. 2002, ApJ, 575, 1144

Vilas, F., Lederer, S. M., Gill, S. L., Jarvis, K. S., \& Thomas-Osip, J. E. 2006, Icarus, 180, 453

Vilenius, E., Kiss, C., Mommert, M., et al. 2012, A\&A, 541, A94

Vilenius, E., Kiss, C., Müller, T., et al. 2014, A\&A, 564, A35

Vincent, J.-B., Bodewits, D., Besse, S., et al. 2015, Nature, 523, 63

Vincent, J.-B., Oklay, N., Pajola, M., et al. 2016, A\&A, 587, A14

Visser, A. E., Richer, J. S., \& Chandler, C. J. 2002, AJ, 124, 2756

Volk, K., \& Malhotra, R. 2008, ApJ, 687, 714

Wada, K., Tanaka, H., Suyama, T., Kimura, H., \& Yamamoto, T. 2009, ApJ, 702, 1490

Wahlberg Jansson, K., \& Johansen, A. 2014, A\&A, 570, A47

Wallis, M. K., \& MacPherson, A. K. 1981, A\&A, 98, 45

Walsh, K. J., Morbidelli, A., Raymond, S. N., O’Brien, D. P., \& Mandell, A. M. 2011, Nature, 475, 206

Weast, R. C. 1974, Handbook of chemistry and physics, 55th edn. (Cleveland: CRC Press)

Weidenschilling, S. J. 1977, MNRAS, 180, 57

Weidenschilling, S. J. 1997, Icarus, 127, 290

Weidenschilling, S. J. 2008, Phys. Scr. T, 130, 014021

Weidenschilling, S. J., Spaute, D., Davis, D. R., \& Marzari, F. 1997, Icarus, 128, 429

Weidling, R., Güttler, C., Blum, J., \& Brauer, F. 2009, ApJ, 696, 2036

Weissman, P. R. 1986, Nature, 320, 242

Weissman, P. R., \& Lowry, S. 2003, Lunar Planet. Sci., 34, 2003

Weissman, P. R., Asphaug, E., \& Lowry, S. C. 2004, in Comets II, eds. M. C.

Festou, U. Keller, \& H. A. Weaver (Tucson: Univ. of Arizona Press), 337

Westphal, A. J., Fakra, S. C., Gainsforth, Z., et al. 2009, ApJ, 694, 18

Wetherill, G. W. 1967, J. Geophys. Res., 72, 2429

Whitman, K., Morbidelli, A., \& Jedicke, R. 2006, Icarus, 183, 101

Wiegert, P., Balam, D., Moss, A., et al. 2007, AJ, 133, 1609

Windmark, F., Birnstiel, T., Güttler, C., et al. 2012a, A\&A, 540, A73

Windmark, F., Birnstiel, T., Ormel, C. W., \& Dullemond, C. P. 2012b, A\&A, 544, L 16

Wood, J. A. 2004, Geochim. Cosmochim. Acta, 68, 4007

Wozniakiewicz, P. J., Ishii, H. A., Kearsley, A. T., et al. 2010, LPSC, 41, 2357

Yabushita, S. 1993, MNRAS, 260, 819

Yamamoto, T. 1985, in Ices in the Solar System, eds. J. Klinger, D. Benest,

A. Doffus, \& R. Smoluchowski (Dordrecht: D. Reidel Publishing Company), 205

Yasui, M., \& Arakawa, M. 2009, J. Geophys. Res., 114, E09004

Youdin, A. N., \& Goodman, J. 2005, ApJ, 620, 459

Zahnle, K., Schenk, P., Levison, H., \& Dones, L. 2003, Icarus, 163, 263 
Zolensky, M. E., Zega, T. J., Yano, H., et al. 2006, Science, 314, 1735

Zsom, A., Ormel, C. W., Güttler, C., Blum, J., \& Dullemond, C. P. 2010, A\&A 513, A57

Zuckerman, B., Foreille, T., \& Kastner, J. H. 1995, Nature, 373, 494

1 Department of Physics and Astronomy, Uppsala University, Box 516, 75120 Uppsala, Sweden

2 Jet Propulsion Laboratory, M/S 183-301, 4800 Oak Grove Drive, Pasadena CA 91109, USA e-mail: bjorn.davidsson@jpl .nasa.gov

3 Max-Planck-Institut für Sonnensystemforschung, Justus-vonLiebig-Weg, 3, 37077 Göttingen, Germany

4 University of Padova, Department of Physics and Astronomy, via Marzolo 8, 35131 Padova, Italy

5 Centro di Ateneo di Studi ed Attivitá Spaziali "Giuseppe Colombo" (CISAS), University of Padova, via Venezia 15, 35131 Padova, Italy

6 PAN Space Research Center, Bartycka 18A, 00716 Warszawa, Poland

7 University of Maryland, Department of Astronomy, College Park, MD 20742-2421, USA

8 Gauss Professor, Akademie der Wissenschaften zu Göttingen and Max-Planck-Institut für Sonnensystemforschung, Justus-vonLiebig-Weg 3, 37077 Göttingen, Germany

9 Aix-Marseille Université, CNRS, LAM (Laboratoire d'Astrophysique de Marseille) UMR 7326, 13388 Marseille, France

10 Physikalisches Institut der Universität Bern, Sidlerstr. 5, 3012 Bern, Switzerland

11 LESIA-Observatoire de Paris, CNRS, Université Pierre et Marie Curie, Université Paris Diderot, 5 place J. Janssen, 92195 Meudon, France

12 Instituto de Astrofísica de Andalucía (CSIC), c/ Glorieta de la Astronomía s/n, 18008 Granada, Spain

13 Institut für Geophysik und extraterrestrische Physik (IGEP), Technische Universität Braunschweig, Mendelssohnstr. 3, 38106 Braunschweig, Germany
14 Dipartimento di Geoscienze, University of Padova, 35131 Padova, Italy

15 Planetary and Space Sciences, Department of Physical Sciences, The Open University, Milton Keynes, MK7 6AA, UK

16 University of Padova, Department of Physics and Astronomy, Vicolo dell'Osservatorio 3, 35122 Padova, Italy

17 Laboratoire d'Astrophysique de Marseille, UMR 7326, CNRS \& Aix-Marseille Université, 38 rue Frédéric Joliot-Curie, 13388 Marseille Cedex 13, France

18 Centro de Astrobiologia, CSIC-INTA, 28850 Torrejon de Ardoz, Madrid, Spain

19 International Space Science Institute, Hallerstraße 6, 3012 Bern, Switzerland

20 Scientific Support Office, European Space Research and Technology Centre/ESA, Keplerlaan 1, Postbus 299, 2201 AZ Noordwijk ZH, The Netherlands

21 LATMOS, CNRS/UVSQ/IPSL, 11 boulevard d'Alembert, 78280 Guyancourt, France

22 INAF, Osservatorio Astronomico di Padova, Vicolo dell'Osservatorio 5, 35122 Padova, Italy

23 CNR-IFN UOS Padova LUXOR, via Trasea, 7, 35131 Padova, Italy

24 Department of Industrial Engineering - University of Padova, via Venezia 1, 35131 Padova, Italy

25 University of Trento, via Mesiano 77, 38100 Trento, Italy

26 Univ. Paris Diderot, Sorbonne Paris Cité, 4 rue Elsa Morante, 75205 Paris Cedex 13, France

27 INAF-Osservatorio Astronomico, via Tiepolo 11, 34014 Trieste, Italy

28 Deutsches Zentrum für Luft - und Raumfahrt (DLR), Institut für Planetenforschung, Rutherfordstraße 2, 12489 Berlin, Germany

29 National Central University, Graduate Institute of Astronomy, 300 Chung-Da Rd, 32054 Chung-Li, Taiwan

30 Operations Department, European Space Astronomy Centre/ESA, PO Box 78, 28691 Villanueva de la Canada, Madrid, Spain

31 University of Padova, Department of Information Engineering, via Gradenigo 6/B, 35131 Padova, Italy 